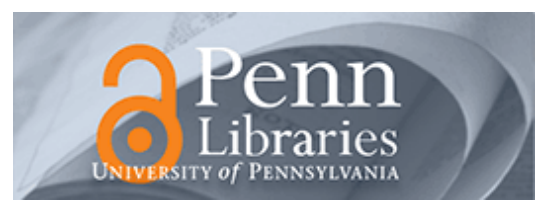

University of Pennsylvania

ScholarlyCommons

\title{
Landau Analysis of the Symmetry of the Magnetic Structure and Magnetoelectric Interaction in Multiferroics
}

\author{
A. Brooks Harris \\ University of Pennsylvania, harris@dept.physics.upenn.edu
}

Follow this and additional works at: https://repository.upenn.edu/physics_papers

Part of the Physics Commons

\section{Recommended Citation}

Harris, A. B. (2007). Landau Analysis of the Symmetry of the Magnetic Structure and Magnetoelectric Interaction in Multiferroics. Retrieved from https://repository.upenn.edu/physics_papers/163

Suggested Citation:

A.B. Harris. (2007) Landau analysis of the symmetry of the magnetic structure and magnetoelectric interaction in multiferroics. Physical Review B 76, 054447.

(C) 2007 The American Physical Society

http://dx.doi.org/10.1103/PhysRevB.76.054447

This paper is posted at ScholarlyCommons. https://repository.upenn.edu/physics_papers/163

For more information, please contact repository@pobox.upenn.edu. 


\title{
Landau Analysis of the Symmetry of the Magnetic Structure and Magnetoelectric Interaction in Multiferroics
}

\author{
Abstract \\ This paper presents a detailed instruction manual for constructing the Landau expansion for \\ magnetoelectric coupling in incommensurate ferroelectric magnets, including $\mathrm{Ni}_{3} \mathrm{~V}_{2} \mathrm{O}_{8}, \mathrm{TbMnO}_{3}, \mathrm{MnWO}_{4}$, \\ $\mathrm{TbMn}_{2} \mathrm{O}_{5}, \mathrm{YMn}_{2} \mathrm{O}_{5}, \mathrm{CuFeO}_{2}$, and $\mathrm{RbFe}\left(\mathrm{MO}_{4}\right)_{2}$. The first step is to describe the magnetic ordering in terms \\ of symmetry adapted coordinates which serve as complex-valued magnetic order parameters whose \\ transformation properties are displayed. In so doing, we use the previously proposed technique to exploit \\ inversion symmetry, since this symmetry has seemingly been universally overlooked. Inversion symmetry \\ severely reduces the number of fitting parameters needed to describe the spin structure, usually by fixing \\ the relative phases of the complex fitting parameters. By introducing order parameters of known \\ symmetry to describe the magnetic ordering, we are able to construct the trilinear magnetoelectric \\ interaction which couples incommensurate magnetic order to the uniform polarization, and thereby we \\ treat many of the multiferroic systems so far investigated. In most cases, the symmetry of the \\ magnetoelectric interaction determines the direction of the magnetically induced spontaneous \\ polarization. We use the Landau description of the magnetoelectric phase transition to discuss the \\ qualitative behavior of various susceptibilities near the phase transition. The consequences of symmetry \\ for optical properties such as polarization induced mixing of Raman and infrared phonons and \\ electromagnons are analyzed. The implication of this theory for microscopic models is discussed. \\ Disciplines \\ Physical Sciences and Mathematics | Physics \\ Comments \\ Suggested Citation: \\ A.B. Harris. (2007) Landau analysis of the symmetry of the magnetic structure and magnetoelectric \\ interaction in multiferroics. Physical Review B 76, 054447. \\ (C) 2007 The American Physical Society \\ http://dx.doi.org/10.1103/PhysRevB.76.054447
}

This journal article is available at ScholarlyCommons: https://repository.upenn.edu/physics_papers/163 


\title{
Landau analysis of the symmetry of the magnetic structure and magnetoelectric interaction in multiferroics
}

\author{
A. B. Harris \\ Department of Physics and Astronomy, University of Pennsylvania, Philadelphia, Pennsylvania 19104, USA
}

(Received 17 February 2007; revised manuscript received 16 May 2007; published 28 August 2007)

\begin{abstract}
This paper presents a detailed instruction manual for constructing the Landau expansion for magnetoelectric coupling in incommensurate ferroelectric magnets, including $\mathrm{Ni}_{3} \mathrm{~V}_{2} \mathrm{O}_{8}, \mathrm{TbMnO}_{3}, \mathrm{MnWO}_{4}, \mathrm{TbMn}_{2} \mathrm{O}_{5}$, $\mathrm{YMn}_{2} \mathrm{O}_{5}, \mathrm{CuFeO}_{2}$, and $\mathrm{RbFe}\left(\mathrm{MO}_{4}\right)_{2}$. The first step is to describe the magnetic ordering in terms of symmetry adapted coordinates which serve as complex-valued magnetic order parameters whose transformation properties are displayed. In so doing, we use the previously proposed technique to exploit inversion symmetry, since this symmetry has seemingly been universally overlooked. Inversion symmetry severely reduces the number of fitting parameters needed to describe the spin structure, usually by fixing the relative phases of the complex fitting parameters. By introducing order parameters of known symmetry to describe the magnetic ordering, we are able to construct the trilinear magnetoelectric interaction which couples incommensurate magnetic order to the uniform polarization, and thereby we treat many of the multiferroic systems so far investigated. In most cases, the symmetry of the magnetoelectric interaction determines the direction of the magnetically induced spontaneous polarization. We use the Landau description of the magnetoelectric phase transition to discuss the qualitative behavior of various susceptibilities near the phase transition. The consequences of symmetry for optical properties such as polarization induced mixing of Raman and infrared phonons and electromagnons are analyzed. The implication of this theory for microscopic models is discussed.
\end{abstract}

DOI: 10.1103/PhysRevB.76.054447

PACS number(s): 75.25.+z, 75.10.Jm, 75.40.Gb

\section{INTRODUCTION}

Recently, there has been increasing interest in systems (multiferroics) which exhibit an observable interaction between magnetic and electric degrees of freedom. ${ }^{1}$ Much interest has centered on a family of multiferroics which display a phase transition in which uniform ferroelectric order appears simultaneously with incommensurate magnetic ordering. Early examples of such a system whose ferroelectric behavior and magnetic structure have been thoroughly studied are terbium manganate, $\mathrm{TbMnO}_{3}$ (TMO), ${ }^{2,3}$ and nickel vanadate, $\mathrm{Ni}_{3} \mathrm{~V}_{2} \mathrm{O}_{8}$ (NVO). ${ }^{4-7}$ A similar comprehensive analysis has recently been given for the triangular lattice compound $\mathrm{RbFe}\left(\mathrm{MoO}_{4}\right)_{2}$ (RFMO). ${ }^{8}$ A number of other systems have been shown to have combined magnetic and ferroelectric transitions, ${ }^{9-14}$ but the investigation of their magnetic structure has been less systematic. Initially, this combined transition was somewhat mysterious, but soon a Landau expansion was developed ${ }^{4}$ to provide a phenomenological explanation of this phenomenon. An alternative picture, similar to an earlier result ${ }^{15}$ based on the concept of a "spin current," and which we refer to as the "spiral formulation," 16 has gained popularity due to its simplicity, but as we will discuss, the Landau theory is more universally applicable and has a number of advantages. The purpose of the present paper is to describe the Landau formulation in the simplest possible terms and to apply it to a large number of currently studied multiferroics. In this way, we hope to demystify this formulation.

It should be noted that this phenomenon (which we call "magnetically induced ferroelectricity") is closely related to the similar behavior of so-called "improper ferroelectrics," which are commonly understood to be the analogous systems in which uniform magnetic order (ferromagnetism or antifer- romagnetism) drives ferroelectricity. ${ }^{17}$ Several decades ago, such systems were studied $^{18}$ and reviewed ${ }^{17,19}$ and present many parallels with the recent developments.

One of the problems one encounters at the outset is how to properly describe the magnetic structure of systems with complicated unit cells. This, of course, is a very old subject, ${ }^{20-22}$ but surprisingly, as will be documented below, the full ramifications of symmetry are not widely known. Accordingly, we feel it necessary to repeat the description of the symmetry analysis of magnetic structures. While the first part of this symmetry analysis is well known to experts, we review it here, especially because our approach is often far simpler and less technical than the standard one. However, either approach lays the groundwork for incorporating the effects of inversion symmetry, which, in the recent literature, have often been overlooked until our analysis of $\mathrm{NVO}^{4-7}$ and TMO. ${ }^{3}$ Inversion symmetry was also addressed by Schweizer with a subsequent correction. ${ }^{23}$ Very recently, a more formal approach to this problem has been given by Radaelli and Chapon ${ }^{24}$ and by Schweizer et al. ${ }^{25}$ However, at least in the simplest cases, the approach initially proposed by us and used here seems easiest. We here apply this formalism to a number of currently studied multiferroics, such as $\mathrm{DyMnO}_{3}$ (DMO), ${ }^{9} \mathrm{MnWO}_{4}$ (MWO), ${ }^{13,14} \mathrm{TbMn}_{2} \mathrm{O}_{5}$ (TMO25), ${ }^{11,12}$ $\mathrm{YMn}_{2} \mathrm{O}_{5}\left(\mathrm{YMO}_{25}\right),{ }^{12} \mathrm{CuFeO}_{2}(\mathrm{CFO}),{ }^{10}$ and $\mathrm{RFMO}^{8}$ As was the case for $\mathrm{NVO}^{4-7}$ and $\mathrm{TMO},{ }^{3}$ once one has in hand the symmetry properties of the magnetic order parameters, one is then able to construct the trilinear magnetoelectric coupling term in the free energy which provides a phenomenological explanation of the combined magnetic and ferroelectric phase transition.

This paper is organized in conformity with the above plan. In Sec. II, we review a simplified version of the symmetry analysis known as representation theory. Here, we 
also review the recently proposed ${ }^{3-7}$ technique to incorporate the consequences of inversion symmetry. In Sec. III, we apply this formalism to develop magnetic order parameters for a number of multiferroic systems, and in Eq. (126) we give a simple example to show how inversion symmetry influences the symmetry of the allowed spin distribution. Then, in Sec. IV, we use the symmetry of the order parameters to construct a magnetoelectric coupling free energy, whose symmetry properties are manifested. We give an analysis of the Landau description of the magnetoelectric phase transition. In particular, we discuss the behavior of various susceptibilities near the phase transition. In Sec. V, we discuss how the magnetoelectric interaction leads to mixing of infrared active and Raman active phonon modes and to the mixing of magnons with phonons. Finally, in Sec. VI, we summarize the results of these calculations and discuss their consequences.

\section{REVIEW OF REPRESENTATION THEORY}

As we shall see, to understand the phenomenology of the magnetoelectric coupling which gives rise to the combined magnetic and ferroelectric phase transition, it is essential to characterize and properly understand the symmetry of the magnetic ordering. In addition, as we shall see, to fully include symmetry restrictions on possible magnetic structures that can be accessed via a continuous phase transition is an extremely powerful aid in the magnetic structure analysis, Accordingly, in this section we review how symmetry considerations restrict the possible magnetic structures which can appear at an ordering transition. The full symmetry analysis has previously been presented elsewhere, ${ }^{3-7}$ but it is useful to repeat it here both to fix the notation and to give the reader convenient access to this analysis which is so essential to the present discussion. To avoid the complexities of the most general form of this analysis (called representation theory), ${ }^{23-25}$ we will limit discussion to systems having some crucial simplifying features. First, we limit consideration to systems in which the magnetic ordering either is incommensurate or equivalent thereto. In the examples we choose, $k$ will usually lie along a symmetry direction of the crystal. Second, we only consider systems which have a center of inversion symmetry, because it is only such systems that have a sharp phase transition at which long-range ferroelectric order appears. Thirdly, we restrict attention to crystals having relatively simple symmetry. (What this means is that except for our discussion of $\mathrm{TbMn}_{2} \mathrm{O}_{5}$, we will consider systems where we do not need the full apparatus of group theory, but can get away with simply labeling the spin functions which describe magnetic order by their eigenvalue under various symmetry operations.) By avoiding the complexities of the most general situations, it is hoped that this paper will be accessible to more readers. Finally, as we will see, it is crucial that the phase transitions we analyze are either continuous or very nearly so. In many of the examples we discuss, our simple approach ${ }^{6}$ is vastly simpler than that of standard representation theory ${ }^{26-28}$ augmented by specialized techniques to explicitly exploit inversion symmetry.

\section{A. Symmetry analysis of the magnetic free energy}

In this section, we give a review of the formalism used previously ${ }^{3,4}$ and presented in detail in Refs. 5-7. Since we are mainly interested in symmetry properties, we will describe the magnetic ordering by a version of mean-field theory in which one writes the magnetic free energy $F_{M}$ as

$$
F_{M}=\frac{1}{2} \sum_{\mathbf{r}, \alpha ; \mathbf{r}^{\prime} \beta} \chi_{\alpha \beta}^{-1}\left(\mathbf{r}, \mathbf{r}^{\prime}\right) S_{\alpha}(\mathbf{r}) S_{\beta}\left(\mathbf{r}^{\prime}\right)+\mathcal{O}\left(S^{4}\right),
$$

where $S_{\alpha}(\mathbf{r})$ is the thermally averaged $\alpha$ component of the spin at position $\mathbf{r}$. In a moment, we will give an explicit approximation for the inverse susceptibility $\chi^{-1}$. We now introduce Fourier transforms in either of two equivalent formulations. In the first formulation (which we refer to as "actual position"), one writes the Fourier transform as

$$
S_{\alpha}(\mathbf{q}, \tau)=N^{-1} \sum_{\mathbf{R}} S_{\alpha}(\mathbf{R}+\boldsymbol{\tau}) e^{i \mathbf{q} \cdot(\mathbf{R}+\tau)}
$$

whereas in the second (which we refer to as "unit cell"), one writes

$$
S_{\alpha}(\mathbf{q}, \tau)=N^{-1} \sum_{\mathbf{R}} S_{\alpha}(\mathbf{R}+\tau) e^{i \mathbf{q} \cdot \mathbf{R}},
$$

where $N$ is the number of unit cells in the system, $\boldsymbol{\tau}$ is the location of the $\pi$ th site within the unit cell, and $\mathbf{R}$ is a lattice vector. Note that in Eq. (2) the phase factor in the Fourier transform is defined in terms of the actual position of the spin rather than in terms of the origin of the unit cell, as is done in Eq. (3). In some cases (viz., NVO), the results are simpler in the actual position formulation, whereas for others (viz., TMO), the unit cell formulation is simpler. We will use whichever formulation is simpler. In either case, the fact that $S_{\alpha}$ has to be real indicates that

$$
S_{\alpha}(-\mathbf{q}, \tau)=S_{\alpha}(\mathbf{q}, \tau)^{*} .
$$

We thus have

$$
F_{M}=\frac{1}{2} \sum_{\mathbf{q} ; \tau, \tau^{\prime}, \alpha, \beta} \chi_{\alpha \beta}^{-1}\left(\mathbf{q} ; \tau, \tau^{\prime}\right) S_{\alpha}(\mathbf{q}, \tau)^{*} S_{\beta}\left(\mathbf{q}, \tau^{\prime}\right)+\mathcal{O}\left(S^{4}\right),
$$

where, for the actual position formulation,

$$
\chi_{\alpha \beta}^{-1}\left(\mathbf{q} ; \tau, \tau^{\prime}\right)=\sum_{\mathbf{R}} \chi_{\alpha \beta}^{-1}\left(\tau, \mathbf{R}+\tau^{\prime}\right) e^{i \mathbf{q} \cdot\left(\mathbf{R}+\tau^{\prime}-\tau\right)},
$$

and for the unit cell formulation,

$$
\chi_{\alpha \beta}^{-1}\left(\mathbf{q} ; \tau, \tau^{\prime}\right)=\sum_{\mathbf{R}} \chi_{\alpha \beta}^{-1}\left(\tau, \mathbf{R}+\tau^{\prime}\right) e^{i \mathbf{q} \cdot \mathbf{R}} .
$$

To make our discussion more concrete, we cite the simplest approximation for a system of spins on an orthorhombic Bravais lattice with general anisotropic exchange coupling so that the Hamiltonian is

$$
\mathcal{H}=\sum_{\alpha, \beta ; \mathbf{r}, \mathbf{r}^{\prime}} J_{\alpha \beta}\left(\mathbf{r}, \mathbf{r}^{\prime}\right) s_{\alpha}(\mathbf{r}) s_{\beta}\left(\mathbf{r}^{\prime}\right)+\sum_{\alpha \mathbf{r}} K_{\alpha} s_{\alpha}(\mathbf{r})^{2},
$$

where $s_{\alpha}(\mathbf{r})$ is the $\alpha$ component of the spin operator at $\mathbf{r}$ and we have included a single ion anisotropy energy assuming 
three inequivalent axes so that the $K_{\alpha}$ are all different. One has that

$$
\chi_{\alpha \beta}^{-1}\left(\mathbf{r}, \mathbf{r}^{\prime}\right)=J_{\alpha \beta}\left(\mathbf{r}, \mathbf{r}^{\prime}\right)+\left[K_{\alpha}+c k T\right] \delta_{\alpha, \beta} \delta_{\mathbf{r}, \mathbf{r}^{\prime}},
$$

where $\delta_{a, b}$ is unity if $a=b$ and is zero otherwise and $c$ is a spin-dependent constant of order unity, so that $-c k \Sigma_{\alpha} S_{\alpha}(\mathbf{r})^{2}$ is the entropy (relative to infinite temperature) associated with a spin $S$. Then,

$$
\begin{aligned}
\chi_{\alpha \beta}^{-1}(\mathbf{q})= & \delta_{\alpha \beta}\left\{2 J_{1}\left[\cos \left(a_{x} q_{x}\right)+\cos \left(a_{y} q_{y}\right)+\cos \left(a_{z} q_{z}\right)\right]+a k T\right. \\
& \left.+K_{\alpha}\right\},
\end{aligned}
$$

where $a_{\alpha}$ is the lattice constant in the $\alpha$ direction $^{29}$ and we assume that $K_{x}<K_{y}<K_{z}$. Graphs of $\chi^{-1}(\mathbf{q})$ are shown in Fig. 1 for both the ferromagnetic $\left(J_{1}<0\right)$ and antiferromagnetic $\left(J_{1}>0\right)$ cases. For the ferromagnetic case, we now introduce a competing antiferromagnetic next-nearest-neighbor (nnn) interaction $J_{2}>0$ along the $x$ axis so that

$$
\begin{aligned}
\chi_{\alpha \alpha}^{-1}\left(q_{x}, q_{y}=\right. & \left.0, q_{z}=0\right)=\left[4 J_{1}+2 J_{1} \cos \left(a_{x} q_{x}\right)+2 J_{2} \cos \left(2 a_{x} q_{x}\right)\right. \\
& \left.+a k T+K_{\alpha}\right],
\end{aligned}
$$

and this is also shown in Fig. 1. As $T$ is lowered, one reaches a critical temperature where one of the eigenvalues of the inverse susceptibility matrix becomes zero. This indicates that the paramagnetic phase is unstable with respect to order corresponding to the critical eigenvector associated with the zero eigenvalue. For the ferromagnet, this happens for zero wave vector, and for the antiferromagnet, for a zone boundary wave vector in agreement with our obvious expectation. For competing interactions, we see that the values of the $J$ 's determine a wave vector at which an eigenvalue of $\chi^{-1}$ is minimal. This is the phenomenon called "wave vector selection," and in this case the selected value of $\mathbf{q}$ is determined by extremizing $\chi^{-1}$ to $\mathrm{be}^{30}$

$$
\cos \left(a_{x} q\right)=J_{1} /\left(4 J_{2}\right),
$$

providing $J_{2}>-J_{1} / 4$. (Otherwise, the system is ferromagnetic.) Note also that crystal symmetry may select a set of symmetry-related wave vectors, which comprise what is known as the star of q. (For instance, if the system were tetragonal, then crystal symmetry would imply that one has the same nnn interactions along the $y$ axis, in which case the system selects a wave vector along the $x$ axis and one of equal magnitude along the $y$ axis.

From the above discussion, it should be clear that if we assume a continuous transition so that the transition is associated with the instability in the terms in the free energy quadratic in the spin amplitudes, then the nature of the ordered phase is determined by the critical eigenvector of the inverse susceptibility, i.e., the eigenvector associated with the eigenvalue of inverse susceptibility which first goes to zero as the temperature is reduced. Accordingly, the aim of this paper is to analyze how crystal symmetry affects the possible forms of the critical eigenvector.

When the unit cell contains $n>1$ spins, the inverse susceptibility for each wave vector $\mathbf{q}$ is a $3 n \times 3 n$ matrix. The ordering transition occurs when, for some selected wave vector(s), an eigenvalue first becomes zero as the temperature is
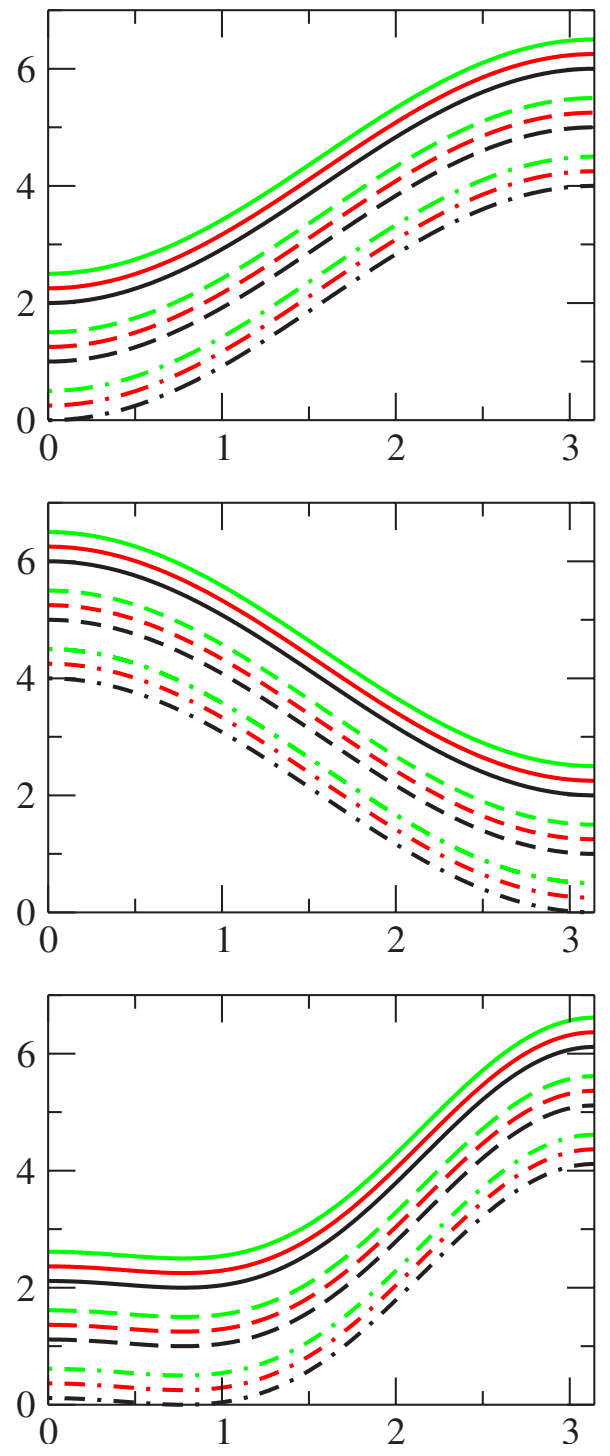

FIG. 1. (Color online) Inverse susceptibility $\chi^{-1}(q, 0,0)$. (top) Ferromagnetic model $\left(J_{1}<0\right)$, (middle) antiferromagnetic model $\left(J_{1}<0\right)$, and (bottom) model with competing interactions (the $\mathrm{nn}$ interaction is antiferromagnetic). In each panel, one sees three groups of curves. Each group consists of the three curves for $\chi_{\alpha \alpha}(q)$ which depend on the component label $\alpha$ due to the anisotropy. The $x$ axis is the easiest axis and the $z$ axis is the hardest. (If the system is orthorhombic, the three axes must all be inequivalent.) The solid curves are for the highest temperature, the dashed curves are for an intermediate temperature, and the dash-dot curves are for $T=T_{c}$, the critical temperature for magnetic ordering. The bottom panel illustrates the nontrivial wave vector selection which occurs when one has competing interactions.

reduced. In the above simple examples involving isotropic exchange interactions, the inverse susceptibility was a $3 \times 3$ diagonal matrix so that each eigenvector trivially has only one nonzero component. The critical eigenvector has spin oriented along the easiest axis, i.e., the one for which $K_{\alpha}$ is minimal. In the present more general case, $n>1$ and arbitrary interactions consistent with crystal symmetry are allowed. To avoid the technicalities of group theory, we use as our guiding principle the fact that the free energy, being an 
TABLE I. General positions (Refs. 33 and 34) within the primitive unit cell for $\mathrm{Cmca}$ which describe the symmetry operations (Ref. 36) of this space group. $2_{\alpha}$ is a twofold rotation (or screw) axis and $m_{\alpha}$ is a mirror (glide) which takes $r_{\alpha}$ into $-r_{\alpha}$ (followed by a translation). All coordinates are expressed as a fraction of lattice parameters so that $x$ really denotes $x a$.

\begin{tabular}{lc}
\hline \hline$E \mathbf{r}=(x, y, z)$ & $2{ }_{c} \mathbf{r}=(\bar{x}, \bar{y}+1 / 2, z+1 / 2)$ \\
$2{ }_{b} \mathbf{r}=(\bar{x}, y+1 / 2, \bar{z}+1 / 2)$ & $2{ }_{a} \mathbf{r}=(x, \bar{y}, \bar{z})$ \\
$\mathcal{I} \mathbf{r}=(\bar{x}, \bar{y}, \bar{z})$ & $m_{c} \mathbf{r}=(x, y+1 / 2, \bar{z}+1 / 2)$ \\
$m_{b} \mathbf{r}=(x, \bar{y}+1 / 2, z+1 / 2)$ & $m_{a} \mathbf{r}=(\bar{x}, y, z)$ \\
\hline \hline
\end{tabular}

expansion in powers of the magnetizations relative to the paramagnetic state, must be invariant under all the symmetry operations of the crystal. ${ }^{26,31}$ This is the same principle that one uses in discussing the symmetry of the electrostatic potential in a crystal. ${ }^{32}$ We now focus our attention on the critically selected wave vector $q$ which has an eigenvalue which first becomes zero as the temperature is lowered. This value of $\mathbf{q}$ is determined by the interactions and we will consider it to be an experimentally determined parameter. Operations which leave the quadratic free energy invariant must leave invariant the term in the free energy $F_{2}(\mathbf{q})$ which involves only the selected wave vector $\mathbf{q}$, namely,

$$
F_{2}(\mathbf{q}) \equiv \frac{1}{2} \sum_{\tau, \tau^{\prime}, \alpha, \beta} \chi_{\alpha \beta}^{-1}\left(\mathbf{q} ; \tau, \tau^{\prime}\right) S_{\alpha}(\mathbf{q}, \tau)^{*} S_{\beta}\left(\mathbf{q}, \tau^{\prime}\right) .
$$

Any symmetry operation takes the original variables before transformation, $S_{\alpha}(\mathbf{q}, \tau)$, into new ones indicated by primes. We write this transformation as

$$
S_{\alpha}^{\prime}(\mathbf{q}, \boldsymbol{\tau})=\sum_{\alpha^{\prime} \tau} U_{\alpha \tau ; \alpha^{\prime} \tau^{\prime}} S_{\alpha^{\prime}}\left(\mathbf{q}, \boldsymbol{\tau}^{\prime}\right)
$$

According to a well known statement of elementary quantum mechanics, if a set of commuting operators $T_{1}, T_{2}, \ldots$ also commutes with $\chi^{-1}(\mathbf{q})$, then the eigenvectors of $\chi^{-1}(\mathbf{q})$ are simultaneously eigenvectors of each of the $T_{i}$ 's. (This much reproduces a well known analysis. ${ }^{20-22}$ We will later consider the effect of inversion, the analysis of which seems to have been universally overlooked). We will apply this simple condition to a number of multiferroic systems currently under investigation. (This approach can be much more straightforward than the standard one when the operations which conserve wave vector unavoidably involve translations.) As a first example, we consider the case of NVO and use the actual position Fourier transforms. In Table I, we give the general positions (this set of positions is the so-called Wyckoff orbit) for the space group Cmca (No. 64 in Ref. 33) of NVO and this table defines the operations of the space group Cmca. In Table II, we list the positions of the two types of sites occupied by the magnetic (Ni) ions, which are called "spine" and "cross-tie" sites in recognition of their distinctive coordination in the lattice, as can be seen in Fig. 2, where we show the conventional unit cell of NVO. Experiments $^{6,38}$ indicate that as the temperature is lowered, the system first develops incommensurate order with $\mathbf{q}$ along the a direction with $q \approx 0.28 .{ }^{39}$ In Fig. 3 we show the phase
TABLE II. Positions (Refs. 34 and 35) of $\mathrm{Ni}^{2+}$ carrying $S=1$ within the primitive unit cell illustrated in Fig. 2. Here, $\mathbf{r}_{s n}$ denotes the position of the $n$th spine site and $\mathbf{r}_{c n}$ that of the $n$th cross-tie site. NVO orders in space group Cmca, so there are six more atoms in the conventional orthorhombic unit cell, which are obtained by a translation through $(0.5 a, 0.5 b, 0)$.
$\mathbf{r}_{s 1}=$
$(0.25,-0.13,0.25)$
$\mathbf{r}_{s 2}=$
$(0.25,0.13,0.75)$
$\mathbf{r}_{s 3}=$
$(0.75,0.13,0.75)$
$\mathbf{r}_{s 4}=$
$(0.75,-0.13,0.25)$
$\mathbf{r}_{c 1}=$
$(0,0,0)$
$\mathbf{r}_{c 2}=$
$(0.5,0,0.5)$

diagram in the $T-H$ plane for $H$ along the c axis, for $T$ $>2 \mathrm{~K}^{6}$

The group of operations which conserve wave vector is generated by (a) the twofold rotation $2_{x}$ and (b) the glide operation $m_{z}$, both of which are defined in Table I. We now discuss how the Fourier spin components transform under various symmetry operations. Here, primed quantities denote the value of the quantity after transformation. Let $\mathcal{O}$

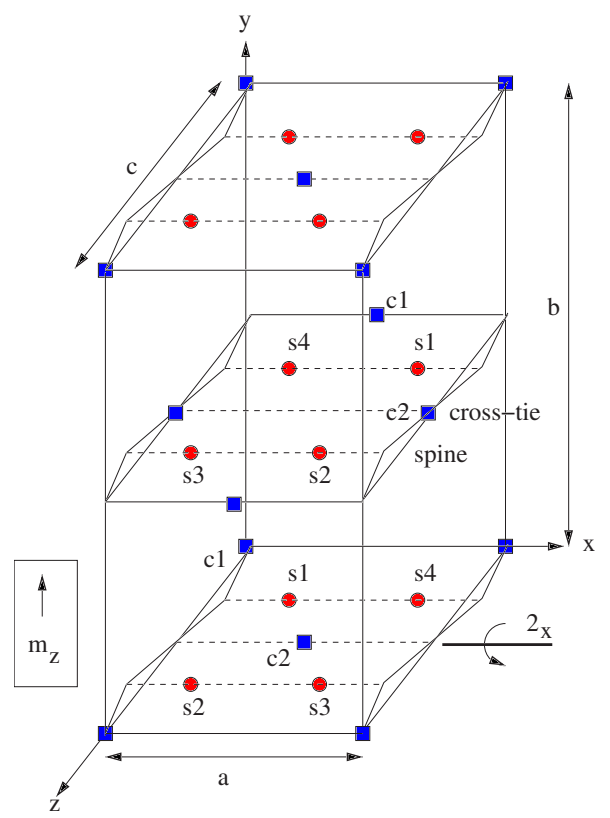

FIG. 2. (Color online) Ni sites in the conventional unit cell of NVO. The primitive translation vectors $\mathbf{v}_{n}$ are $\mathbf{v}_{1}=(a / 2) \hat{a}+(b / 2) \hat{b}$, $\mathbf{v}_{2}=(a / 2) \hat{a}-(b / 2) \hat{b}$, and $\mathbf{v}_{3}=c \hat{c}$. The "cross-tie" sites blue online $\mathrm{c} 1$ and $\mathrm{c} 2$ lie in a plane with $b=0$. The "spine" sites red online are labeled s1, s2, s3, and s4 and they may be visualized as forming chains parallel to the a axis. These chains are in the buckled plane with $b= \pm \delta$, where $\delta=0.13 b$ as is indicated. Cross-tie sites in adjacent planes are displaced by $( \pm b / 2) \hat{b}$. Spine sites in adjacent planes are located directly above (or below) the sites in the plane shown. In the incommensurate phases, the wave vector describing magnetic ordering lies along the a axis. The axis of the twofold rotation about the $x$ axis is shown. The glide plane is indicated by the mirror plane at $z=\frac{3}{4}$ and the arrow above $m_{z}$ indicates that a translation of $b / 2$ in the $y$ direction is involved. 


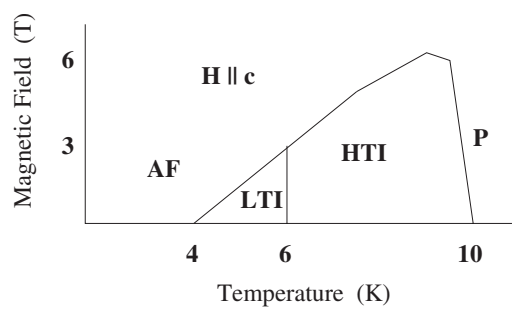

FIG. 3. Schematic phase diagram for NVO for a magnetic field applied along the $\mathbf{c}$ direction, taken from Ref. 6. Here, AF is an antiferromagnetic phase with a weak ferromagnetic moment, $\mathrm{P}$ is the paramagnetic phase, HTI is the "high-temperature incommensurate" phase in which the moments are essentially aligned along the a axis with a sinusoidally modulated amplitude (according to irrep $\Gamma_{4}$ ), and LTI is the "low-temperature incommensurate" phase in which transverse order along the $\mathbf{b}$ axis appears to make an elliptically polarized order-parameter wave (according to irreps $\Gamma_{4}$ and $\Gamma_{1}$ ). A spontaneous polarization $\mathbf{P}$ appears only in the LTI phase with $\mathbf{P}$ along $\mathbf{b}$.

$\equiv \mathcal{O}_{s} \mathcal{O}_{r}$ be a symmetry operation which we decompose into operations on the spin $\mathcal{O}_{s}$ and on the position $\mathcal{O}_{r}$. The effect of transforming a spin by such an operator is to replace the spin at the "final" position $\mathbf{R}_{f}$ by the transformed spin which initially was at the position $\mathcal{O}_{r}^{-1} \mathbf{R}_{f}$. So, we write

$$
S_{\alpha}^{\prime}\left(\mathbf{R}_{f}, \boldsymbol{\tau}_{f}\right)=\mathcal{O}_{s} S_{\alpha}\left(\mathcal{O}_{r}^{-1}\left[\mathbf{R}_{f}, \boldsymbol{\tau}_{f}\right]\right)=\xi_{\alpha}\left(\mathcal{O}_{s}\right) S_{\alpha}\left(\mathbf{R}_{i}, \boldsymbol{\tau}_{i}\right),
$$

where the subscripts $i$ and $f$ denote initial and final values and $\xi_{\alpha}\left(\mathcal{O}_{s}\right)$ is the factor introduced by $\mathcal{O}_{s}$ for a pseudovector, namely,

$$
\begin{gathered}
\xi_{x}\left(2_{x}\right)=1, \quad \xi_{y}\left(2_{x}\right)=\xi_{z}\left(2_{x}\right)=-1, \\
\xi_{x}\left(m_{z}\right)=\xi_{y}\left(m_{z}\right)=-1, \quad \xi_{z}\left(m_{z}\right)=1 .
\end{gathered}
$$

Note that $\mathcal{O} S_{\alpha}(\mathbf{R}, \boldsymbol{\tau})$ is not the result of applying $\mathcal{O}$ to move and reorient the spin at $\mathbf{R}+\boldsymbol{\tau}$, but instead is the value of the spin at $\mathbf{R}+\boldsymbol{\tau}$ after the spin distribution is acted upon by $\mathcal{O}$. Thus, for actual position Fourier transforms, we have

$$
\begin{aligned}
S_{\alpha}^{\prime}\left(\mathbf{q}, \boldsymbol{\tau}_{f}\right) & =N^{-1} \sum_{\mathbf{R}} S_{\alpha}^{\prime}\left(\mathbf{R}_{f}, \boldsymbol{\tau}_{f}\right) e^{i \mathbf{q} \cdot\left(\mathbf{R}_{f}+\tau_{f}\right)} \\
& =\xi_{\alpha}\left(\mathcal{O}_{s}\right) N^{-1} \sum_{\mathbf{R}} S_{\alpha}\left(\mathbf{R}_{i}, \boldsymbol{\tau}_{i}\right) e^{i \mathbf{q} \cdot\left(\mathbf{R}_{f}+\tau_{f}\right)} \\
& =\xi_{\alpha}\left(\mathcal{O}_{s}\right) S_{\alpha}\left(\mathbf{q}, \boldsymbol{\tau}_{i}\right) e^{i \mathbf{q} \cdot\left[\mathbf{R}_{f}+\tau_{f}-\mathbf{R}_{i}-\tau_{i}\right]}
\end{aligned}
$$

We may write this as

$$
\mathcal{O} S_{\alpha}\left(\mathbf{q}, \boldsymbol{\tau}_{f}\right)=\xi_{\alpha}\left(\mathcal{O}_{s}\right) S_{\alpha}\left(\mathbf{q}, \boldsymbol{\tau}_{i}\right) e^{i \mathbf{q} \cdot\left[\mathbf{R}_{f}+\boldsymbol{\tau}_{f}-\mathbf{R}_{i}-\boldsymbol{\tau}_{i}\right]}
$$

This formulation may not be totally intuitive, because one is tempted to regard the operation $\mathcal{O}$ acting on a spin at an initial location and taking it (and perhaps reorienting it) to another location. Here, instead, we consider the spin distribution and how the transformed distribution at a location is related to the distribution at the initial location.

Similarly, the result for unit cell Fourier transforms is

$$
S_{\alpha}^{\prime}\left(\mathbf{q}, \boldsymbol{\tau}_{f}\right)=\xi_{\alpha}\left(\mathcal{O}_{s}\right) S_{\alpha}\left(\mathbf{q}, \boldsymbol{\tau}_{i}\right) e^{i \mathbf{q} \cdot\left[\mathbf{R}_{f}-\mathbf{R}_{i}\right]}
$$

As before, we may write this as

$$
\mathcal{O} S_{\alpha}\left(\mathbf{q}, \boldsymbol{\tau}_{f}\right)=\xi_{\alpha}\left(\mathcal{O}_{s}\right) S_{\alpha}\left(\mathbf{q}, \boldsymbol{\tau}_{i}\right) e^{i \mathbf{q} \cdot\left[\mathbf{R}_{f}-\mathbf{R}_{i}\right]} .
$$

Under transformation by inversion, $\xi_{\alpha}(\mathcal{I})=1$ and

$$
\begin{aligned}
S_{\alpha}^{\prime}\left(\mathbf{q}, \boldsymbol{\tau}_{f}\right)^{*} & =N^{-1} \sum_{\mathbf{R}} S_{\alpha}\left(\mathbf{R}_{i}, \boldsymbol{\tau}_{i}\right) e^{-i \mathbf{q} \cdot\left(\mathbf{R}_{f}+\tau_{f}\right)} \\
& =S_{\alpha}\left(\mathbf{q}, \boldsymbol{\tau}_{i}\right) e^{i \mathbf{q} \cdot\left[-\mathbf{R}_{f}-\tau_{f}-\mathbf{R}_{i}-\boldsymbol{\tau}_{i}\right]}=S_{\alpha}\left(\mathbf{q}, \boldsymbol{\tau}_{i}\right)
\end{aligned}
$$

for actual position Fourier transforms. For unit cell transforms, we get

$$
S_{\alpha}^{\prime}\left(\mathbf{q}, \boldsymbol{\tau}_{f}\right)^{*}=S_{\alpha}\left(\mathbf{q}, \boldsymbol{\tau}_{i}\right) e^{i \mathbf{q} \cdot\left[-\mathbf{R}_{f}-\mathbf{R}_{i}\right]}=S_{\alpha}\left(\mathbf{q}, \boldsymbol{\tau}_{i}\right) e^{i \mathbf{q} \cdot\left[\boldsymbol{\tau}_{f}+\boldsymbol{\tau}_{i}\right]} .
$$

Now, we apply this formalism to find the actual position Fourier coefficients which are eigenfunctions of the two operators $2_{x}$ and $m_{z}$. In so doing, note the simplicity of Eq. (17): since, for NVO, the operations $2_{x}$ and $m_{z}$ do not change the $x$ coordinate, we simply have

$$
S_{\alpha}^{\prime}\left(\mathbf{q}, \boldsymbol{\tau}_{f}\right)=\xi_{\alpha} S_{\alpha}^{\prime}\left(\mathbf{q}, \boldsymbol{\tau}_{i}\right) .
$$

Thus, the eigenvalue conditions for $2_{x}$ acting on the spine sites (1-4) are

$$
\begin{aligned}
& S_{\alpha}(q, 1)^{\prime}=\xi_{\alpha}\left(2_{x}\right) S_{\alpha}(q, 2)=\lambda\left(2_{x}\right) S_{\alpha}(q, 1), \\
& S_{\alpha}(q, 2)^{\prime}=\xi_{\alpha}\left(2_{x}\right) S_{\alpha}(q, 1)=\lambda\left(2_{x}\right) S_{\alpha}(q, 2), \\
& S_{\alpha}(q, 3)^{\prime}=\xi_{\alpha}\left(2_{x}\right) S_{\alpha}(q, 4)=\lambda\left(2_{x}\right) S_{\alpha}(q, 3), \\
& S_{\alpha}(q, 4)^{\prime}=\xi_{\alpha}\left(2_{x}\right) S_{\alpha}(q, 3)=\lambda\left(2_{x}\right) S_{\alpha}(q, 4),
\end{aligned}
$$

from which we see that $\lambda\left(2_{x}\right)= \pm 1$ and

$$
\begin{aligned}
& S_{\alpha}(q, 2)=\left[\xi_{\alpha}\left(2_{x}\right) / \lambda\left(2_{x}\right)\right] S_{\alpha}(q, 1), \\
& S_{\alpha}(q, 3)=\left[\xi_{\alpha}\left(2_{x}\right) / \lambda\left(2_{x}\right)\right] S_{\alpha}(q, 4) .
\end{aligned}
$$

The eigenvalue conditions for $m_{z}$ acting on the spine sites are

$$
\begin{aligned}
& S_{\alpha}(q, 1)^{\prime}=\xi_{\alpha}\left(m_{z}\right) S_{\alpha}(q, 4)=\lambda\left(m_{z}\right) S_{\alpha}(q, 1), \\
& S_{\alpha}(q, 4)^{\prime}=\xi_{\alpha}\left(m_{z}\right) S_{\alpha}(q, 1)=\lambda\left(m_{z}\right) S_{\alpha}(q, 4), \\
& S_{\alpha}(q, 2)^{\prime}=\xi_{\alpha}\left(m_{z}\right) S_{\alpha}(q, 3)=\lambda\left(m_{z}\right) S_{\alpha}(q, 2), \\
& S_{\alpha}(q, 3)^{\prime}=\xi_{\alpha}\left(m_{z}\right) S_{\alpha}(q, 2)=\lambda\left(m_{z}\right) S_{\alpha}(q, 3),
\end{aligned}
$$

from which we see that $\lambda\left(m_{z}\right)= \pm 1$ and

$$
S_{\alpha}(q, 4)=\left[\xi_{\alpha}\left(m_{z}\right) / \lambda\left(m_{z}\right)\right] S_{\alpha}(q, 1) .
$$

We thereby construct the wave functions for the spine sites which are simultaneously eigenvectors of $2_{x}$ and $m_{z}$ and these are given in Table III. The results for the cross-tie sites are obtained in the same way and are also given in the table. Each set of eigenvalues corresponds to a different symmetry label [irreducible representation (irrep)], here denoted $\Gamma_{n}$. Since each operator can have either of two eigenvalues, we have four symmetry labels to consider. Note that these spin 
TABLE III. Allowed spin functions (i.e., actual position Fourier coefficients) within the unit cell of NVO for wave vector $(q, 0,0)$ which are eigenvectors of $2_{x}$ and $m_{z}$ with the eigenvalues $\lambda$ listed. Inversion symmetry is not yet taken into account. Each of the four combinations of eigenvalues represents a different symmetry, which we identify with a symmetry label $\Gamma_{n}$. In group theoretical language, $\Gamma_{n}$ is referred to as an irreducible representation (irrep), for which we use the notation of Ref. $6 . n(\Gamma)$ is the number of independent structure parameters in the wave function having the symmetry label $\Gamma$. Group theory indicates that $n(\Gamma)$ is the number of times the irrep $\Gamma$ is contained in the original (18-dimensional) representation corresponding to $S_{\alpha}(\mathbf{q}, \tau)$. For the labeling of the sites, $\tau$ is as in Table II and Fig. 2. Here, $n_{p}^{\alpha}(p=\mathrm{s}$ or c, $\alpha=a, b, c)$ denotes the complex quantity $n_{p}^{\alpha}(\mathbf{q})$.

\begin{tabular}{|c|c|c|c|c|}
\hline Irrep & $\Gamma_{1}$ & $\Gamma_{2}$ & $\Gamma_{3}$ & $\Gamma_{4}$ \\
\hline$\lambda\left(2_{x}\right)$ & +1 & +1 & -1 & -1 \\
\hline$\lambda\left(m_{z}\right)$ & +1 & -1 & -1 & +1 \\
\hline$n(\Gamma)$ & 4 & 4 & 5 & 5 \\
\hline \multirow[t]{3}{*}{$\mathbf{S}(\mathbf{q}, s 1)$} & $n_{s}^{a}$ & $n_{s}^{a}$ & $n_{s}^{a}$ & $n_{s}^{a}$ \\
\hline & $n_{s}^{b}$ & $n_{s}^{b}$ & $n_{s}^{b}$ & $n_{s}^{b}$ \\
\hline & $n_{s}^{c}$ & $n_{s}^{c}$ & $n_{s}^{c}$ & $n_{s}^{c}$ \\
\hline \multirow[t]{3}{*}{$\mathbf{S}(\mathbf{q}, s 2)$} & $n_{s}^{a}$ & $n_{s}^{a}$ & $-n_{s}^{a}$ & $-n_{s}^{a}$ \\
\hline & $-n_{s}^{b}$ & $-n_{s}^{b}$ & $n_{s}^{b}$ & $n_{s}^{b}$ \\
\hline & $-n_{s}^{c}$ & $-n_{s}^{c}$ & $n_{s}^{c}$ & $n_{s}^{c}$ \\
\hline \multirow[t]{3}{*}{$\mathbf{S}(\mathbf{q}, s 3)$} & $-n_{s}^{a}$ & $n_{s}^{a}$ & $-n_{s}^{a}$ & $n_{s}^{a}$ \\
\hline & $n_{s}^{b}$ & $-n_{s}^{b}$ & $n_{s}^{b}$ & $-n_{s}^{b}$ \\
\hline & $-n_{s}^{c}$ & $n_{s}^{c}$ & $-n_{s}^{c}$ & $n_{s}^{c}$ \\
\hline \multirow[t]{3}{*}{$\mathbf{S}(\mathbf{q}, s 4)$} & $-n_{s}^{a}$ & $n_{s}^{a}$ & $n_{s}^{a}$ & $-n_{s}^{a}$ \\
\hline & $-n_{s}^{b}$ & $n_{s}^{b}$ & $n_{s}^{b}$ & $-n_{s}^{b}$ \\
\hline & $n_{s}^{c}$ & $-n_{s}^{c}$ & $-n_{s}^{c}$ & $n_{s}^{c}$ \\
\hline \multirow[t]{3}{*}{$\mathbf{S}(\mathbf{q}, c 1)$} & $n_{c}^{a}$ & $n_{c}^{a}$ & 0 & 0 \\
\hline & 0 & 0 & $n_{c}^{b}$ & $n_{c}^{b}$ \\
\hline & 0 & 0 & $n_{c}^{c}$ & $n_{c}^{c}$ \\
\hline \multirow[t]{3}{*}{$\mathbf{S}(\mathbf{q}, c 2)$} & $-n_{c}^{a}$ & $n_{c}^{a}$ & 0 & 0 \\
\hline & 0 & 0 & $n_{c}^{b}$ & $-n_{c}^{b}$ \\
\hline & 0 & 0 & $-n_{c}^{c}$ & $n_{c}^{c}$ \\
\hline
\end{tabular}

functions, since they are actually Fourier coefficients, are complex-valued quantities. [The spin itself is real because $F(-\mathbf{q})=F(\mathbf{q})^{*}$.] Each column of Table III gives the most general form of an allowed eigenvector for which one has $n(\Gamma)=4$ or $n(\Gamma)=5$ (depending on the irrep) independent complex constants. In terms of the amplitude $X_{\Gamma}^{(m)}(\mathbf{q})$ of the $m$ th eigenfunction of irrep $\Gamma$ (at wave vector q) and the corresponding eigenvalue $\lambda_{\Gamma}^{(m)}(\mathbf{q})$, the free energy is diagonal:

$$
F_{2}=\frac{1}{2} \sum_{\mathbf{q}} \sum_{\Gamma} \sum_{m=1}^{n(\Gamma)} \lambda_{\Gamma}^{(m)}(\mathbf{q})\left|X_{\Gamma}^{(m)}(\mathbf{q})\right|^{2} .
$$

These eigenvalues can be identified as the inverse susceptibility associated with "normal modes" of spin configurations.
To further illustrate the meaning of this table, we explicitly write, in Eq. (48) below, the spin distribution arising from one irrep, $\Gamma_{4}$. These spin functions are schematically shown for the spine sites in Fig. 16 below. Here, our main interest is in the mode which first becomes unstable as the temperature is lowered.

So far, the present analysis reproduces the standard results and indeed computer programs exist to construct such tables. However, for multiferroics it may be quicker to obtain and understand how to construct the possible spin functions by hand rather than to understand how to use the program. Usually, these programs give the results in terms of unit cell Fourier transforms, which we claim are not as natural a representation in cases like NVO. In terms of unit cell Fourier transforms, the eigenvalue conditions for $2_{x}$ acting on the spine sites (1-4) are the same as Eq. (24) for actual position Fourier transforms because the operation $2_{x}$ does not change the unit cell. However, for the glide operation $m_{z}$, this is not the case. If we start from site 1 or site 2 , the translation along the $y$ axis takes the spin to a final unit cell displaced by $(-a / 2) \hat{i}+(b / 2) \hat{j}$, whereas if we start from site 3 or site 4 , the translation along the $y$ axis takes the spin to a final unit cell displaced by $(a / 2) \hat{i}+(b / 2) \hat{j}$. Now, the eigenvalue conditions for $m_{z}$ acting on the spine sites (1-4) are

$$
\begin{aligned}
& S_{\alpha}(q, 1)^{\prime}=\xi_{\alpha}\left(m_{z}\right) S_{\alpha}(q, 4) \eta=\lambda\left(m_{z}\right) S_{\alpha}(q, 1), \\
& S_{\alpha}(q, 4)^{\prime}=\xi_{\alpha}\left(m_{z}\right) S_{\alpha}(q, 1) \eta^{*}=\lambda\left(m_{z}\right) S_{\alpha}(q, 4), \\
& S_{\alpha}(q, 2)^{\prime}=\xi_{\alpha}\left(m_{z}\right) S_{\alpha}(q, 3) \eta=\lambda\left(m_{z}\right) S_{\alpha}(q, 2), \\
& S_{\alpha}(q, 3)^{\prime}=\xi_{\alpha}\left(m_{z}\right) S_{\alpha}(q, 2) \eta^{*}=\lambda\left(m_{z}\right) S_{\alpha}(q, 3),
\end{aligned}
$$

where $\eta=\exp (i \pi q)$. One finds that all entries for $S(\mathbf{q}, s 3)$, $S(\mathbf{q}, s 4)$, and $S(\mathbf{q}, c 2)$ now carry the phase factor $\eta^{*}=\exp (-i \pi q)$. However, this is just the factor to make the unit cell result

$$
\mathbf{S}(\mathbf{R}, \boldsymbol{\tau})=\mathbf{S}(\mathbf{q}, \boldsymbol{\tau}) e^{-i \mathbf{q} \cdot \mathbf{R}}
$$

be the same (to within an overall phase factor) as the actual position result

$$
\mathbf{S}(\mathbf{R}, \boldsymbol{\tau})=\mathbf{S}(\mathbf{q}, \boldsymbol{\tau}) e^{-i \mathbf{q} \cdot(\mathbf{R}+\boldsymbol{\tau})} .
$$

We should emphasize that in such a simple case as NVO, it is actually not necessary to invoke any group theoretical concepts to arrive at the results of Table III for the most general spin distribution consistent with crystal symmetry.

More importantly, it is not commonly understood ${ }^{20-22}$ that one can also extract information using the symmetry of an operation (inversion) which does not conserve wave vector. ${ }^{3-7,23-25}$ Since what we are about to say may be unfamiliar, we start from first principles. The quadratic free energy may be written as

$$
F_{2}=\sum_{\mathbf{q}} \sum_{\tau, \tau^{\prime} ; \alpha \beta} F_{\alpha \beta}^{\tau \tau^{\prime}} S_{\alpha}(\mathbf{q}, \tau)^{*} S_{\beta}\left(\mathbf{q}, \tau^{\prime}\right),
$$

where we restrict the sum over wave vectors to the star of the wave vector of interest. One term of this sum is 


$$
F_{2}\left(\mathbf{q}_{0}\right)=\sum_{\tau, \tau^{\prime} ; \alpha \beta} F_{\alpha \beta}^{\tau \tau^{\prime}} S_{\alpha}\left(\mathbf{q}_{0}, \tau\right)^{*} S_{\beta}\left(\mathbf{q}_{0}, \tau^{\prime}\right) .
$$

It should be clear that the quadratic free energy $F_{2}$ is invariant under all the symmetry operations of the paramagnetic space group (i.e., what one calls the space group of the crystal). ${ }^{26,31}$ For centrosymmetric crystals, there are three classes of such symmetry operations. The first class consists of those operations which leave $\mathbf{q}_{0}$ invariant and these are the symmetries taken into account in the usual formulation. ${ }^{20-22}$ The second class consists of operations which take $\mathbf{q}_{0}$ into another wave vector of the star (call it $\mathbf{q}_{1}$ ), where $\mathbf{q}_{1} \neq-\mathbf{q}_{0}$. Use of these symmetries allows one to completely characterize the wave in function at wave vector $\mathbf{q}_{1}$ in terms of the wave function for $\mathbf{q}_{0}$. These relations are needed if one is to discuss the possibility of simultaneously condensing more than one wave vector in the star of $\mathbf{q}^{28,40}$ Finally, the third class consists of spatial inversion (unless the wave vector and its negative differ by a reciprocal lattice vector, in which case inversion belongs in class 1). The role of inversion symmetry is almost universally overlooked, ${ }^{20-22}$ as is evident from examination of a number of recent papers. Unlike the operations of class 1 which takes $S_{n}(\mathbf{q})$ into an $S_{n^{\prime}}(\mathbf{q})$ (for irreps of dimension 1 which is true for most cases considered in this paper), inversion takes $S_{n}(\mathbf{q})$ into an $S_{n^{\prime}}(-\mathbf{q})$. Nevertheless, it does take the free energy written in Eq. (33) into itself and restricts the possible form of the wave functions. So, we now consider the consequences of invariance of $F_{2}$ under inversion. ${ }^{3-7}$ For this purpose, we write Eq. (13) in terms of the spin coordinates $\mathbf{n}$ of Table III. (The result will, of course, depend on which symmetry label $\Gamma$ we consider.) In any case, the part of $F_{2}$ which depends on $\mathbf{q}_{0}$ can be written as

$$
\begin{aligned}
F_{2}\left(\mathbf{q}_{0}\right) & =\sum_{\tau, \tau^{\prime} ; \alpha \beta} F_{\alpha \beta}^{\tau \tau^{\prime}} S_{\alpha}\left(\mathbf{q}_{0}, \tau\right)^{*} S_{\beta}\left(\mathbf{q}_{0}, \tau^{\prime}\right) \\
& =\sum_{N, \alpha ; N^{\prime}, \beta ; \Gamma} G_{N, \alpha ; N^{\prime}, \beta}\left[n_{N}^{\alpha}(\Gamma)\right]^{*}\left[n_{N^{\prime}}^{\beta}(\Gamma)\right],
\end{aligned}
$$

where $N$ and $N^{\prime}$ assume the values $s$ for spin and $c$ for cross-tie and $\alpha$ and $\beta$ label components, and the sums over $N$ and $\alpha$ (and similarly $N^{\prime}$ and $\beta$ ) are over the $n(\Gamma)$ variables needed to specify the wave function associated with the symmetry label (irrep) $\Gamma$. From now on, we keep only the terms belonging to the irrep which is active and for notational simplicity we leave the corresponding argument $\Gamma$ of $\mathbf{n}$ implicit. Then, we see that invariance under inversion implies that

$$
\begin{aligned}
F_{2}(\mathbf{q}) & =\sum_{N, \alpha ; N^{\prime}, \beta} G_{N, \alpha ; N^{\prime}, \beta}\left[n_{N}^{\alpha}\right]^{*} n_{N^{\prime}}^{\beta} \\
& =\sum_{N, \alpha ; N^{\prime}, \beta} G_{N, \alpha ; N^{\prime}, \beta}\left[\mathcal{I} n_{N}^{\alpha}\right]^{*}\left[\mathcal{I} n_{N^{\prime}}^{\beta}\right] .
\end{aligned}
$$

Now, we need to understand the effect of $\mathcal{I}$ on the spin Fourier coefficients listed in Table III. Since we use actual position Fourier coefficients, we apply Eq. (21). For the cross-tie variables (which sit at a center of inversion symmetry), inversion takes the spin coordinates of one spine sublattice into the complex conjugate of itself:
TABLE IV. The same as Table III for NVO) except that now the effect of inversion symmetry is taken into account, as a result of which, apart from an overall phase factor, all the $n$ 's in this table can be taken to be real valued.

\begin{tabular}{lcccc}
\hline \hline Irrep & $\Gamma_{1}$ & $\Gamma_{2}$ & $\Gamma_{3}$ & $\Gamma_{4}$ \\
\hline$\lambda\left(2_{x}\right)$ & +1 & +1 & -1 & -1 \\
$\lambda\left(m_{z}\right)$ & +1 & -1 & -1 & +1 \\
\hline $\mathbf{S}(\mathbf{q}, s 1)$ & $i n_{s}^{a}$ & $n_{s}^{a}$ & $i n_{s}^{a}$ & $n_{s}^{a}$ \\
& $n_{s}^{b}$ & $i n_{s}^{b}$ & $n_{s}^{b}$ & $i n_{s}^{b}$ \\
& $i n_{s}^{c}$ & $n_{s}^{c}$ & $i n_{s}^{c}$ & $n_{s}^{c}$ \\
\hline $\mathbf{S}(\mathbf{q}, s 2)$ & $i n_{s}^{a}$ & $n_{s}^{a}$ & $-i n_{s}^{a}$ & $-n_{s}^{a}$ \\
& $-n_{s}^{b}$ & $-i n_{s}^{b}$ & $n_{s}^{b}$ & $i n_{s}^{b}$ \\
& $-i n_{s}^{c}$ & $-n_{s}^{c}$ & $i n_{s}^{c}$ & $n_{s}^{c}$ \\
\hline $\mathbf{S}(\mathbf{q}, s 3)$ & $-i n_{s}^{a}$ & $n_{s}^{a}$ & $-i n_{s}^{a}$ & $n_{s}^{a}$ \\
& $n_{s}^{b}$ & $-i n_{s}^{b}$ & $n_{s}^{b}$ & $-i n_{s}^{b}$ \\
& $-i n_{s}^{c}$ & $n_{s}^{c}$ & $-i n_{s}^{c}$ & $n_{s}^{c}$ \\
\hline $\mathbf{S}(\mathbf{q}, s 4)$ & $-i n_{s}^{a}$ & $n_{s}^{a}$ & $i n_{s}^{a}$ & $-n_{s}^{a}$ \\
& $-n_{s}^{b}$ & $i n_{s}^{b}$ & $n_{s}^{b}$ & $-i n_{s}^{b}$ \\
& $i n_{s}^{c}$ & $-n_{s}^{c}$ & $-i n_{s}^{c}$ & $n_{s}^{c}$ \\
\hline $\mathbf{S}(\mathbf{q}, c 1)$ & $n_{c}^{a}$ & $n_{c}^{a}$ & 0 & 0 \\
& 0 & 0 & $n_{c}^{b}$ & $n_{c}^{b}$ \\
& 0 & 0 & $n_{c}^{c}$ & $n_{c}^{c}$ \\
\hline $\mathbf{S}(\mathbf{q}, c 2)$ & $-n_{c}^{a}$ & $n_{c}^{a}$ & 0 & 0 \\
& 0 & 0 & $n_{c}^{b}$ & $-n_{c}^{b}$ \\
& 0 & 0 & $-n_{c}^{c}$ & $n_{c}^{c}$ \\
\hline \hline & & & &
\end{tabular}

$$
\mathcal{I S}(\mathbf{q}, c n)=[\mathbf{S}(\mathbf{q}, c n)]^{*} .
$$

Thus, in terms of the $n$ 's this gives

$$
\mathcal{I} n_{c}^{\alpha}=\left[n_{c}^{\alpha}\right]^{*}, \quad \alpha=x, y, z .
$$

The effect of inversion on the spine variables again follows from Eq. (21). Since inversion interchanges sublattices 1 and 3 , we have

$$
[\mathbf{S}(\mathbf{q}, s 3)]^{\prime}=[\mathbf{S}(\mathbf{q}, s 1)]^{*} .
$$

For $\lambda\left(2_{x}\right)=\lambda\left(m_{z}\right)=+1$ (i.e., for irrep $\left.\Gamma_{1}\right)$, we substitute the values of the spin vectors from the first column of Table III to get

$$
\begin{gathered}
\mathcal{I}\left[-n_{s}^{a}\right]=\left[n_{s}^{a}\right]^{*}, \quad \mathcal{I}\left[n_{s}^{b}\right]=\left[n_{s}^{b}\right]^{*}, \\
\mathcal{I}\left[-n_{s}^{c}\right]=\left[n_{s}^{c}\right]^{*} .
\end{gathered}
$$

Note that some components introduce a factor -1 under inversion and others do not. (Which ones have the minus signs depends on which irrep we consider.) If we make a change of variable by replacing $n_{s}^{\alpha}$ in column 1 of Table III by $i \widetilde{n}_{s}^{\alpha}$ for those components for which $\mathcal{I}$ introduces a minus sign and replacing the other $n_{s}^{\alpha}$ by $\widetilde{n}_{s}^{\alpha}$, then we may rewrite the first column of Table III in the form given in Table IV. We re- 
place all the cross-tie variables $n_{x}^{\alpha}$ by $\tilde{n}_{x}^{\alpha}$. In terms of these new tilde variables, one has

$$
\mathcal{I}\left[\tilde{n}_{s}^{\alpha}\right]=\left[\tilde{n}_{s}^{\alpha}\right]^{*} .
$$

(It is convenient to define the spin Fourier coefficients so that they all transform in the same way under inversion. Otherwise, one would have to keep track of variables which transform with a plus sign and those which transform with a minus sign.) Repeating this process for all the other irreps, we write the possible spin functions as those of Table IV. We give an explicit formula for the spin distribution for one irrep in Eq. (48) below.

Now, we implement Eq. (35), where the spin functions are taken to be the variables listed in Table IV. First, note that the matrix $\mathbf{G}$ in Eq. (35) has to be Hermitian to ensure that $F_{2}$ is real:

$$
G_{M, \alpha ; N, \beta}=\left[G_{N, \beta ; M, \alpha}\right]^{*} .
$$

Then, using Eq. (40), we find that Eq. (35) is

$$
\begin{aligned}
F_{2}\left(\mathbf{q}_{0}\right) & =\sum_{M, \alpha ; N, \beta}\left[\tilde{n}_{M}^{\alpha}\right]^{*} G_{M, \alpha ; N, \beta} \tilde{n}_{N}^{\beta} \\
& =\sum_{M, \alpha ; N, \beta}\left[\mathcal{I} \tilde{n}_{M}^{\alpha}\right]^{*} G_{M, \alpha ; N, \beta}\left[\mathcal{I} \tilde{n}_{N}^{\beta}\right] \\
& =\sum_{M, \alpha ; N, \beta} \tilde{n}_{M}^{\alpha} G_{M, \alpha ; N, \beta}\left[\tilde{n}_{N}^{\beta}\right]^{*}=\sum_{M, \alpha ; N, \beta}\left[\tilde{n}_{M}^{\alpha}\right]^{*} G_{N, \beta ; M, \alpha}\left[\tilde{n}_{N}^{\beta}\right],
\end{aligned}
$$

where, in the last line, we interchanged the roles of the dummy indices $M, \alpha$ and $N, \beta$. By comparing the first and last lines, one sees that the matrix $G$ is symmetric. Since this matrix is also Hermitian, all its elements must be real valued. Thus, all its eigenvectors can be taken to have only realvalued components. However, the $m$ 's are allowed to be complex valued. So, the conclusion is that for each irrep, we may write

$$
\tilde{n}_{N}^{\alpha}(\Gamma)=e^{i \phi_{\Gamma}}\left[r_{N}^{\alpha}(\Gamma)\right],
$$

where the $r$ 's are all real valued and $\phi_{\Gamma}$ is an overall phase which can be chosen arbitrarily for each $\Gamma$. When only a single irrep is active, it is likely that the phase will be fixed by high-order umklapp terms in the free energy, but the effects of such phase locking may be beyond the range of experiments. $^{41}$

It is worth noting how these results should be (and in a few cases $^{3,4,6}$ have been) used in the structure determinations. One should choose the best fit to the diffraction data using, in turn, each irrep (i.e., each set of eigenvalues of $2_{x}$ and $m_{z}$ ). Within each irrep, one parametrizes the spin structure by choosing the Fourier coefficients as in the relevant column of Table IV. Note that instead of having four or five complex coefficients to describe the six sites within the unit cell (see Table III), one has only four or five (depending on the representation) real-valued coefficients to determine. The relative phases of the complex coefficients have all been fixed by invoking inversion symmetry. This is clearly a significant step in increasing the precision of the determination of the magnetic structure from experimental data.

\section{B. Order parameters}

We now review how the above symmetry classification influences the introduction of order parameters which allow the construction of Landau expansions. ${ }^{4,6}$ The form of the order parameter should be such that it has the potential to describe all ordering which are allowed by the quadratic free energy $F_{2}$. Thus, for an isotropic Heisenberg model on a cubic lattice, the order parameter has three components (i.e., it involves a three-dimensional irrep) because although the fourth order terms will restrict order to occur only along certain directions, as far as the quadratic terms are concerned, all directions are equivalent. The analogy here is that the overall phase of the spin function $\phi(\Gamma)$ is not fixed by the quadratic free energy and accordingly the order parameter must be a complex variable which includes such a phase. One also recognizes that although the amplitude of the critical eigenvector is not fixed by the quadratic terms in the free energy, the ratios of its components are fixed by the specific form of the inverse susceptibility matrix. Although we do not wish to discuss the explicit form of this matrix, what should be clear is that the components of the spins which order must be proportional to the components of the critical eigenvector. The actual amplitude of the spin ordering is determined by the competition between the quadratic and fourth-order terms in the free energy. If $\Gamma_{p}$ is the irrep which is critical, then just below the ordering temperature we write

$$
\tilde{n}_{N}^{\alpha}(\mathbf{q})=\boldsymbol{\sigma}_{p}(\mathbf{q}) r_{N}^{\alpha}\left(\Gamma_{p}\right),
$$

where the $r$ 's are real components of the critical eigenvector (associated with the critical eigenvalue of irrep $\Gamma_{p}$ ) of the matrix $\mathbf{G}$ of Eq. (35) and are now normalized by

$$
\sum_{\alpha N}\left[r_{N}^{\alpha}\right]^{2}=1
$$

Here, the order parameter for irrep $\Gamma(\mathbf{q}), \boldsymbol{\sigma}_{p}(\mathbf{q})$, is a complex variable, since it has to incorporate the arbitrary complex phase $\phi_{p}$ associated with irrep $\Gamma_{p}$ :

$$
\boldsymbol{\sigma}_{p}( \pm|\mathbf{q}|)=\sigma_{p} e^{\mp i \phi_{p}} .
$$

The order parameter transforms as indicated in the tables by its listed eigenvalues under the symmetry operations $2_{x}$ and $m_{z}$. Since the components of the critical eigenvector are dominantly determined by the quadratic terms, ${ }^{42}$ one can say that just below the ordering temperature the description in terms of an order parameter continues to hold but

$$
\sigma_{p} \sim\left|T_{c}-T\right|^{\beta_{p}},
$$

where mean-field theory gives $\beta=1 / 2$ but corrections due to fluctuation are expected. ${ }^{43}$

To summarize and illustrate the use of Table IV, we write an explicit expression for the magnetizations assuming the active irrep to be $\Gamma_{4}\left[\lambda\left(2_{x}\right)=-1\right.$ and $\left.\lambda\left(m_{z}\right)=+1\right]$. We use the definition of the order parameter and sum over both signs of the wave vector to get

$$
\begin{aligned}
& S_{x}(\mathbf{r}, s 1)=2 \sigma_{4} r_{s}^{x} \cos \left(q x+\phi_{4}\right), \\
& S_{y}(\mathbf{r}, s 1)=2 \sigma_{4} r_{s}^{y} \sin \left(q x+\phi_{4}\right),
\end{aligned}
$$




$$
\begin{aligned}
& S_{z}(\mathbf{r}, s 1)=2 \sigma_{4} r_{s}^{z} \cos \left(q x+\phi_{4}\right), \\
& S_{x}(\mathbf{r}, s 2)=-2 \sigma_{4} r_{s}^{x} \cos \left(q x+\phi_{4}\right), \\
& S_{y}(\mathbf{r}, s 2)=2 \sigma_{4} r_{s}^{y} \sin \left(q x+\phi_{4}\right), \\
& S_{z}(\mathbf{r}, s 2)=2 \sigma_{4} r_{s}^{z} \cos \left(q x+\phi_{4}\right), \\
& S_{x}(\mathbf{r}, s 3)=2 \sigma_{4} r_{s}^{x} \cos \left(q x+\phi_{4}\right), \\
& S_{y}(\mathbf{r}, s 3)=-2 \sigma_{4} r_{s}^{y} \sin \left(q x+\phi_{4}\right), \\
& S_{z}(\mathbf{r}, s 3)=2 \sigma_{4} r_{s}^{z} \cos \left(q x+\phi_{4}\right), \\
& S_{x}(\mathbf{r}, s 4)=-2 \sigma_{4} r_{s}^{x} \cos \left(q x+\phi_{4}\right), \\
& S_{y}(\mathbf{r}, s 4)=-2 \sigma_{4} r_{s}^{y} \sin \left(q x+\phi_{4}\right), \\
& S_{z}(\mathbf{r}, s 4)=2 \sigma_{4} r_{s}^{z} \cos \left(q x+\phi_{4}\right), \\
& S_{x}(\mathbf{r}, c 1)=0, \\
& S_{y}(\mathbf{r}, c 1)=2 \sigma_{4} r_{c}^{y} \cos \left(q x+\phi_{4}\right), \\
& S_{z}(\mathbf{r}, c 1)=2 \sigma_{4} r_{c}^{z} \cos \left(q x+\phi_{4}\right), \\
& S_{x}(\mathbf{r}, c 1)=0 \\
& S_{y}(\mathbf{r}, c 2)=-2 \sigma_{4} r_{c}^{y} \cos \left(q x+\phi_{4}\right), \\
& S_{z}(\mathbf{r}, c 2)=2 \sigma_{4} r_{c}^{z} \cos \left(q x+\phi_{4}\right),
\end{aligned}
$$

and similarly for the other irreps. [The observed magnetic structures are described qualitatively in the caption of Fig. 3. The actual values of the structure parameters $r_{x}^{\alpha}$ in Eq. (48) and its analog for irrep $\Gamma_{1}$ are given in Ref. 6.] Here, $\mathbf{r}$ $\equiv(x, y, z)$ is the actual location of the spin. Using explicit expressions like the above (or more directly from Table IV), one can verify that the order parameters $\left(\boldsymbol{\sigma}_{p}\right.$ for irrep $\left.\Gamma_{p}\right)$ have the transformation properties

$$
\begin{array}{ll}
2_{x} \boldsymbol{\sigma}_{1}(\mathbf{q})=+\boldsymbol{\sigma}_{1}(\mathbf{q}), & m_{z} \boldsymbol{\sigma}_{1}(\mathbf{q})=+\boldsymbol{\sigma}_{1}(\mathbf{q}), \\
2_{x} \boldsymbol{\sigma}_{2}(\mathbf{q})=+\boldsymbol{\sigma}_{2}(\mathbf{q}), & m_{z} \boldsymbol{\sigma}_{2}(\mathbf{q})=-\boldsymbol{\sigma}_{2}(\mathbf{q}), \\
2_{x} \boldsymbol{\sigma}_{3}(\mathbf{q})=-\boldsymbol{\sigma}_{3}(\mathbf{q}), & m_{z} \boldsymbol{\sigma}_{3}(\mathbf{q})=-\boldsymbol{\sigma}_{3}(\mathbf{q}), \\
2_{x} \boldsymbol{\sigma}_{4}(\mathbf{q})=-\boldsymbol{\sigma}_{4}(\mathbf{q}), & m_{z} \boldsymbol{\sigma}_{4}(\mathbf{q})=+\boldsymbol{\sigma}_{4}(\mathbf{q}),
\end{array}
$$

and

$$
\mathcal{I} \boldsymbol{\sigma}_{n}(\mathbf{q})=\left[\boldsymbol{\sigma}_{n}(\mathbf{q})\right]^{*}
$$

Note that even when more than a single irrep is present, the introduction of order parameters, as done here, provides a framework within which one can represent the spin distribution as a linear combination of distributions each having a characteristic symmetry, as expressed by Eq. (49). When the structure of the unit cell is ignored, ${ }^{16}$ that information is not readily accessible. Also note that the phase of each irrep $\Gamma_{n}$ is defined so that when $\phi_{n}=0$, the wave is inversion symmetric about $\mathbf{r}=0$. When $\phi_{n}$ is nonzero, it is possible to invoke the incommensurability to find a lattice site which is arbitrarily close to a center of inversion symmetry of the mathematical spin function. Thus, each irrep has a center of inversion symmetry whose location is implicitly defined by the value of $\phi_{n}$. When only a single irrep is active, the specification of $\phi_{n}$ is not important. However, when one has two irreps, then inversion symmetry is only maintained if the centers of inversion symmetry of the two irreps coincide, i.e., if their phases are equal.

In many systems, the initial incommensurate order that first occurs as the temperature is lowered becomes unstable as the temperature is further lowered. ${ }^{30}$ Typically, the initial order involves spins oriented along their easy axis with sinusoidally varying magnitude. However, the fourth-order terms in the Landau expansion (which we have not written explicitly) favor fixed length spins. As the temperature is lowered, the fixed length constraint becomes progressively more important, and at a second, lower, critical temperature a transition occurs in which transverse components become nonzero. Although the situation is more complicated when there are several spins per unit cell, the result is similar: the fixed length constraint is best realized when more than a single irrep has condensed. So, for NVO and TMO, as the temperature is lowered one encounters a second phase transition in which a second irrep appears. Within a low-order Landau expansion, this phenomenon is described by a free energy of the form ${ }^{6}$

$F=\frac{1}{2}\left(T-T_{>}\right) \sigma_{>}^{2}+\frac{1}{2}\left(T-T_{<}\right) \sigma_{<}^{2}+u_{>} \sigma_{>}^{4}+u_{<} \sigma_{<}^{4}+w \sigma_{>}^{2} \sigma_{<}^{2}$,

where $T_{>}>T_{<}$. This system has been studied in detail by Bruce and Aharony. ${ }^{44}$ For our purposes, the most important result is that for suitable values of the parameters, ordering in $\sigma_{>}$occurs at $T_{>}$and at a lower temperature (when $T-T_{<}$ $\left.+2 w \sigma_{>}^{2}=0\right)$ order in $\sigma_{<}$may occur. The application of this theory to the present situation is simple: we can (and usually do) have two magnetic phase transitions in which, first, one irrep and then at a lower temperature a second irrep condense. A question arises as to whether the condensation of one irrep can induce the condensation of a second irrep. This is not possible because the two irreps have different symmetries. However, could the presence of two irreps $\Gamma_{>}$and $\Gamma_{<}$ induce the appearance of a third irrep $\Gamma_{3}$ at the temperature at which $\Gamma_{<}$first appears? For that to happen would require that $\Gamma_{>}^{n} \otimes \Gamma_{<}^{m} \otimes \Gamma_{3}$ contain the unit representation for some values of $n$ and $m$. This or any higher combination of representations is not allowed for the simple four irreps system like NVO. In more complex systems, one might have to allow for such a phenomenon.

\section{APPLICATIONS}

In this section, we apply the above formalism to a number of multiferroics of current interest. 
TABLE V. General positions for space group $P 2 / c$.

\begin{tabular}{ll}
\hline \hline$E \mathbf{r}=(x, y, z)$ & $m_{y} \mathbf{r}=\left(x, \bar{y}, z+\frac{1}{2}\right)$ \\
$\mathcal{I} \mathbf{r}=(\bar{x}, \bar{y}, \bar{z})$ & $2_{y} \mathbf{r}=\left(\bar{x}, y, \bar{z}+\frac{1}{2}\right)$
\end{tabular}

\section{A. $\mathrm{MnWO}_{4}$}

$\mathrm{MnWO}_{4}$ (MWO) crystallizes in the space group $P 2 / c$ (No. 14 in Ref. 33) whose general positions are given in Table V. The two magnetic Mn ions per unit cell are at positions

$$
\boldsymbol{\tau}_{1}=\left(\frac{1}{2}, y, \frac{1}{4}\right), \quad \boldsymbol{\tau}_{2}=\left(\frac{1}{2}, 1-y, \frac{3}{4}\right) .
$$

The wave vector of incommensurate magnetic ordering is $^{45} \mathbf{q}=\left(q_{x}, 1 / 2, q_{z}\right)$, with $q_{x} \approx-0.21$ and $q_{z} \approx 0.46$, and is left invariant by the identity and $m_{y}$. We start by constructing the eigenvectors of the quadratic free energy (i.e., the inverse susceptibility matrix). Here, we use unit cell Fourier transforms to facilitate comparison with Ref. 45. Below, $X, Y$, and $Z$ denote integers (in units of lattice constants). When

$$
\mathbf{R}_{f}+\boldsymbol{\tau}_{f}=(X, Y, Z)+\boldsymbol{\tau}_{1}=\left(X+\frac{1}{2}, Y+y, Z+\frac{1}{4}\right)
$$

and

$$
\begin{aligned}
\mathbf{R}_{i}+\boldsymbol{\tau}_{i} & =\left[m_{y}\right]^{-1}\left(\mathbf{R}_{f}+\boldsymbol{\tau}_{f}\right)=\left(X+\frac{1}{2},-Y-y, Z-\frac{1}{4}\right) \\
& =(X,-Y-1, Z-1)+\boldsymbol{\tau}_{2},
\end{aligned}
$$

then Eq. (19) gives the eigenvalue condition for $m_{y}$ to be

$$
\begin{aligned}
S_{\alpha}^{\prime}\left(\mathbf{q}, \tau_{1}\right) & =\xi_{\alpha}\left(m_{y}\right) S_{\alpha}\left(\mathbf{q}, \boldsymbol{\tau}_{2}\right) e^{2 \pi i \mathbf{q} \cdot[(2 Y+1) \hat{j}+\hat{k}]} \\
& =\xi_{\alpha}\left(m_{y}\right) S_{\alpha}\left(\mathbf{q}, \tau_{2}\right) e^{\pi i+2 \pi i q_{z}}=\lambda S_{\alpha}\left(\mathbf{q}, \tau_{1}\right),
\end{aligned}
$$

where $\xi_{x}\left(m_{y}\right)=-\xi_{y}\left(m_{y}\right)=\xi_{z}\left(m_{y}\right)=-1$. When

$$
\mathbf{R}_{f}+\boldsymbol{\tau}_{f}=(X, Y, Z)+\boldsymbol{\tau}_{2}=\left(X+\frac{1}{2}, Y+1-y, Z+\frac{3}{4}\right),
$$

then

$$
\mathbf{R}_{i}+\boldsymbol{\tau}_{i}=\left(X+\frac{1}{2},-Y-1+y, Z+\frac{1}{4}\right)=(X,-Y-1, Z)+\boldsymbol{\tau}_{1},
$$

and Eq. (19) gives the eigenvalue condition to be

$$
\begin{aligned}
S_{\alpha}^{\prime}\left(\mathbf{q}, \tau_{2}\right) & =\xi_{\alpha}\left(m_{y}\right) S_{\alpha}\left(\mathbf{q}, \boldsymbol{\tau}_{1}\right) e^{2 \pi i \mathbf{q} \cdot(2 Y+1) \hat{j}}=\xi_{\alpha}\left(m_{y}\right) S_{\alpha}\left(\mathbf{q}, \tau_{1}\right)[-1] \\
& =\lambda S_{\alpha}\left(\mathbf{q}, \tau_{2}\right) .
\end{aligned}
$$

From Eqs. (55) and (58), we get $\lambda= \pm e^{i \pi q_{z}}$ and

$$
S_{\alpha}\left(\mathbf{q}, \tau_{2}\right)=-\left[\xi_{\alpha}\left(m_{y}\right) / \lambda\right] S_{\alpha}\left(\mathbf{q}, \tau_{1}\right) .
$$

So, we get the results listed in Table VI.
TABLE VI. Allowed spin eigenfunctions for MWO (apart from an overall phase factor) before inversion symmetry is taken into account, where $a=\exp \left(-i \pi q_{z} / 2\right)$. Here, the $n(\mathbf{q})$ 's are complex and we have taken the liberty to adjust the overall phase to give a symmetrical looking result. However, these results are equivalent to Table II of Ref. 45.

\begin{tabular}{lcc}
\hline \hline Irrep & $\Gamma_{1}$ & $\Gamma_{2}$ \\
$\lambda\left(m_{y}\right)$ & $e^{i \pi q_{z}}$ & $-e^{i \pi q_{z}}$ \\
$\mathbf{S}(\mathbf{q}, 1)$ & $a^{*} n_{x}$ & $a^{*} n_{x}$ \\
& $a^{*} n_{y}$ & $a^{*} n_{y}$ \\
& $a^{*} n_{z}$ & $a^{*} n_{z}$ \\
& $a n_{x}$ & $-a n_{x}$ \\
$\mathbf{S}(\mathbf{q}, 2)$ & $-a n_{y}$ & $a n_{y}$ \\
& $a n_{z}$ & $-a n_{z}$ \\
\hline \hline
\end{tabular}

So far, the analysis is essentially the completely standard one. Now, we use the fact that the free energy is invariant under spatial inversion, even though that operation does not conserve wave vector. ${ }^{3,4,6,7}$ We now determine the effect of inversion on the $n$ 's. As will become apparent, use of unit cell Fourier transforms makes this analysis more complicated than if we had used actual position transforms. We use Eq. (22) to write

$$
\mathcal{I} S(\mathbf{q}, \tau=1)=S(\mathbf{q}, \tau=2)^{*} e^{-2 \pi i \mathbf{q} \cdot(\hat{i}+\hat{j}+\hat{k})} \equiv b S(\mathbf{q}, 2)^{*},
$$

where $b=-\exp \left[-2 \pi i\left(q_{x}+q_{z}\right)\right]$. For $\Gamma_{2}$, we get

$$
\mathcal{I}\left[n_{x}, n_{y}, n_{z}\right]=\left[-n_{x}, n_{y},-n_{z}\right]^{*} b,
$$

which we can write as

$$
\mathcal{I} n_{\alpha}=b \xi_{\alpha}\left(m_{y}\right) n_{\alpha}^{*}
$$

Now, the free energy is quadratic in the Fourier spin coefficients, which are linearly related to the $n$ 's. So, the free energy can be written as

$$
F_{2}=\mathbf{n}^{\dagger} \mathbf{G n}
$$

where $\mathbf{n}=\left(n_{x}, n_{y}, n_{z}\right)$ is a column vector and $\mathbf{G}$ is a $3 \times 3$ matrix which we write as

$$
\mathbf{G}=\left[\begin{array}{lll}
A & \alpha & \beta \\
\alpha^{*} & B & \gamma \\
\beta^{*} & \gamma^{*} & C
\end{array}\right],
$$

where, for Hermiticity, the Roman letters are real and the Greek ones complex. Now, we use the fact that we must also have invariance with respect to inversion, which after all is a crystal symmetry. Thus,

$$
F_{2}=[\mathcal{I} \mathbf{n}]^{\dagger} \mathbf{G}[\mathcal{I} \mathbf{n}]
$$

This can be written as 
TABLE VII. The same as Table VI (for TMO) except that here inversion symmetry is taken into account. Here, $r, s$, and $t$ are real. All six components can be multiplied by an overall phase factor which we have not been explicitly written.

\begin{tabular}{lcc}
\hline \hline Irrep & $\Gamma_{1}$ & $\Gamma_{2}$ \\
$\lambda\left(m_{y}\right)$ & $e^{i \pi q_{z}}$ & $-e^{i \pi q_{z}}$ \\
$\mathbf{S}(\mathbf{q}, 1)$ & $a^{*} r$ & $-i a^{*} r$ \\
& $i a^{*} s$ & $a^{*} s$ \\
& $a^{*} t$ & $-i a^{*} t$ \\
$\mathbf{S}(\mathbf{q}, 2)$ & $a r$ & $i a r$ \\
& $-i a s$ & as \\
& at & $i a t$ \\
\hline \hline
\end{tabular}

$$
\begin{aligned}
F_{2} & =\sum_{\alpha \beta} b^{*} \xi_{\alpha}\left(m_{y}\right) n_{\alpha} G_{\alpha \beta} b \xi_{\beta}\left(m_{y}\right) n_{\beta}^{*} \\
& =\sum_{\alpha \beta} \xi_{\alpha}\left(m_{y}\right) n_{\alpha} G_{\alpha \beta} \xi_{\beta}\left(m_{y}\right) n_{\beta}^{*} .
\end{aligned}
$$

Thus, we may write

$$
F_{2}=\mathbf{n}^{\operatorname{tr}}\left[\begin{array}{ccc}
A & -\alpha & \beta \\
-\alpha^{*} & B & -\gamma \\
\beta^{*} & -\gamma^{*} & C
\end{array}\right] \mathbf{n}^{*}=\mathbf{n}^{\dagger}\left[\begin{array}{ccc}
A & -\alpha^{*} & \beta^{*} \\
-\alpha & B & -\gamma^{*} \\
\beta & -\gamma & C
\end{array}\right] \mathbf{n},
$$

where "tr" indicates transpose (so $\mathbf{n}^{\text {tr }}$ is a row vector). Since the two expressions for $F_{2}$, Eqs. (63) and (67), must be equal, we see that $\alpha=i a, \beta=b$, and $\gamma=i c$, where $a, b$, and $c$ must be real. Thus, $\mathbf{G}$ is of the form

$$
\mathbf{G}=\left[\begin{array}{ccc}
A & i a & b \\
-i a & B & i c \\
b & -i c & C
\end{array}\right]
$$

where all the letters are real. This means that the critical eigenvector describing the long-range order has to be of the form

$$
\left(n_{x}, n_{y}, n_{z}\right)=e^{i \phi}(r, i s, t),
$$

where $r, s$, and $t$ are real. For $\Gamma_{2}$, we set $e^{i \phi}=-i$. For $\Gamma_{1}$, we set $e^{i \phi}=1$. (These choices are not essential. They just make the symmetry more obvious.) Thus, we obtain the final results given in Table VII. Lautenschlager et al. ${ }^{45}$ say (just above Table II) "Depending on the choice of the amplitudes and phases..." What we see here is that inversion symmetry fixes the phases without the possibility of a choice (just as it did for NVO). Note again that we have about half the variables to fix in a structure determination when we take advantage of inversion invariance to fix the phase of the complex structure constants.

\section{Order parameter}

Now, we discuss the definition of the order parameter for this system. For this purpose, we replace $r$ by $\boldsymbol{\sigma} r, s$ by $\boldsymbol{\sigma} s$, etc., with the normalization that

$$
r^{2}+s^{2}+t^{2}=1
$$

Here, the order parameter $\boldsymbol{\sigma}$ is complex because we always have the freedom to multiply the wave function by a phase factor. (This phase factor might be "locked" by higher-order terms in the free energy, but we do not consider that phenomenon here. ${ }^{46}$ ) We record the symmetry properties of the order parameter. With our choice of phases, we have

$$
\begin{gathered}
\mathcal{I} \boldsymbol{\sigma}_{n}(\mathbf{q})=\left[\boldsymbol{\sigma}_{n}(\mathbf{q})\right]^{*}, \\
m_{y} \boldsymbol{\sigma}_{n}(\mathbf{q})=\lambda\left(\Gamma_{n}\right) \boldsymbol{\sigma}_{n}(\mathbf{q}), \\
m_{y} \boldsymbol{\sigma}_{n}(-\mathbf{q})=\lambda\left(\Gamma_{n}\right)^{*} \boldsymbol{\sigma}_{n}(-\mathbf{q}),
\end{gathered}
$$

where $\boldsymbol{\sigma}_{n}(\mathbf{q}) \equiv \sigma_{n} e^{-i \phi_{n}}$ is the complex-valued order parameter for ordering of irrep $\Gamma_{n}$ and $\lambda\left(\Gamma_{n}\right)$ is the eigenvalue of $m_{y}$ given in Table VII. Now, we write an explicit formula for the spin distribution in terms of the order parameters of the two irreps:

$$
\begin{aligned}
\mathbf{S}(\mathbf{R}, \tau= & 1)=2 \sigma_{1}\left[\left(r_{1} \hat{i}+t_{1} \hat{k}\right) \cos \left(\mathbf{q} \cdot \mathbf{R}+\phi_{1}-\pi q_{z} / 2\right)\right. \\
& \left.+s_{1} \hat{j} \sin \left(\mathbf{q} \cdot \mathbf{R}+\phi_{1}-\pi q_{z} / 2\right)\right] \\
& +2 \sigma_{2}\left[\left(-r_{2} \hat{i}-t_{2} \hat{k}\right) \sin \left(\mathbf{q} \cdot \mathbf{R}+\phi_{2}-\pi q_{z} / 2\right)\right. \\
& \left.+s_{2} \hat{j} \cos \left(\mathbf{q} \cdot \mathbf{R}+\phi_{2}-\pi q_{z} / 2\right)\right], \\
\mathbf{S}(\mathbf{R}, \tau= & 2)=2 \sigma_{1}\left[\left(r_{1} \hat{i}+t_{1} \hat{k}\right) \cos \left(\mathbf{q} \cdot \mathbf{R}+\phi_{1}+\pi q_{z} / 2\right)\right. \\
& \left.-s_{1} \hat{j} \sin \left(\mathbf{q} \cdot \mathbf{R}+\phi_{1}+\pi q_{z} / 2\right)\right] \\
& +2 \sigma_{2}\left[\left(r_{2} \hat{i}+t_{2} \hat{k}\right) \sin \left(\mathbf{q} \cdot \mathbf{R}+\phi_{2}+\pi q_{z} / 2\right)\right. \\
& \left.+s_{2} \hat{j} \cos \left(\mathbf{q} \cdot \mathbf{R}+\phi_{2}+\pi q_{z} / 2\right)\right] .
\end{aligned}
$$

One can explicitly verify that these expressions are consistent with Eq. (71). Note that when only one of the order parameters (say, $\sigma_{n}$ ) is nonzero, we have inversion symmetry with respect to a redefined origin where $\phi_{n}=0$. For each irrep, we have to specify three real parameters, $\sigma r_{n}, \sigma s_{n}$, and $\sigma t_{n}$, and one overall phase, $\phi_{n}$, rather than three complexvalued parameters had we not invoked inversion symmetry.

\section{B. $\mathrm{TbMnO}_{3}$}

Here, we give the full details of the calculations for $\mathrm{TbMnO}_{3}$ described in Ref. 3. The presentation here differs cosmetically from that in Ref. 5. The space group of $\mathrm{TbMnO}_{3}$ is $\mathrm{Pbnm}$ which is No. 62 in Ref. 33 (although the positions are listed there for the Pnma setting). The space group operations for a general Wyckoff orbit are given in Table VIII. In Table IX, we list the positions of the Mn and $\mathrm{Tb}$ ions within the unit cell and these are also shown in Fig. 4. The phase diagram for magnetic fields up to $14 \mathrm{~T}$ along the a axis is shown in Fig. 5.

To start, we study the operations that leave invariant the wave vector of the incommensurate phase which first orders as the temperature is lowered. Experimentally, ${ }^{49}$ this wave vector is found to be $(0, q, 0)$, with ${ }^{39} q \approx 0.28$. These relevant operators (see Table VIII) are $m_{x}$ and $m_{z}$. We follow the 
TABLE VIII. General positions for Pbnm. Notation is the same as in Table I.

\begin{tabular}{lc}
\hline \hline$E \mathbf{r}=(x, y, z)$ & $2_{x} \mathbf{r}=\left(x+\frac{1}{2}, \bar{y}+\frac{1}{2}, \bar{z}\right)$ \\
$2_{z} \mathbf{r}=\left(\bar{x}, \bar{y}, z+\frac{1}{2}\right)$ & $2_{y} \mathbf{r}=\left(\bar{x}+\frac{1}{2}, y+\frac{1}{2}, \bar{z}+\frac{1}{2}\right)$ \\
$\mathcal{I} \mathbf{r}=(\bar{x}, \bar{y}, \bar{z})$ & $m_{x} \mathbf{r}=\left(\bar{x}+\frac{1}{2}, y+\frac{1}{2}, z\right)$ \\
$m_{z} \mathbf{r}=\left(x, y, \bar{z}+\frac{1}{2}\right)$ & $m_{y} \mathbf{r}=\left(x+\frac{1}{2}, \bar{y}+\frac{1}{2}, z+\frac{1}{2}\right)$ \\
\hline \hline
\end{tabular}

approach used for MWO, but use "actual location" Fourier transforms. We set $\mathbf{R}_{f}+\boldsymbol{\tau}_{f} \equiv \mathbf{r}$ in order to use Eq. (17) and we need to evaluate

$$
\begin{aligned}
\Lambda & \equiv \exp \left\{2 \pi i \mathbf{q} \cdot\left[\mathbf{r}-\left(m_{x}\right)^{-1} \mathbf{r}\right]\right\}=\exp \left\{2 \pi i q \hat{j} \cdot\left[y \hat{j}-\left(m_{x}\right)^{-1} y \hat{j}\right]\right\} \\
& =e^{i \pi q}
\end{aligned}
$$

and

$$
\begin{aligned}
\Lambda^{\prime} & \equiv \exp \left\{2 \pi i \mathbf{q} \cdot\left[\mathbf{r}-\left(m_{z}\right)^{-1} \mathbf{r}\right]\right\}=\exp \left\{2 \pi q \hat{j} \cdot\left[y \hat{j}-\left(m_{z}\right)^{-1} y \hat{j}\right]\right\} \\
& =1
\end{aligned}
$$

We list in Table $\mathrm{X}$ the transformation table of sublattice indices of TMO.

Therefore, the eigenvalue condition for transformation by $m_{x}$ is

$$
S_{\alpha}^{\prime}\left(\mathbf{q}, \tau_{f}\right)=\xi_{\alpha}\left(m_{x}\right) S_{\alpha}\left(\mathbf{q}, \tau_{i}\right) \Lambda=\lambda\left(m_{x}\right) S_{\alpha}\left(\mathbf{q}, \tau_{f}\right)
$$

and that for transformation by $m_{z}$ is

$$
S_{\alpha}^{\prime}\left(\mathbf{q}, \tau_{f}\right)=\xi_{\alpha}\left(m_{z}\right) S_{\alpha}\left(\mathbf{q}, \tau_{i}\right)=\lambda\left(m_{z}\right) S_{\alpha}\left(\mathbf{q}, \tau_{f}\right),
$$

where $\xi_{x}\left(m_{x}\right)=-\xi_{y}\left(m_{x}\right)=-\xi_{z}\left(m_{x}\right)=1$ and $\xi_{\alpha}\left(m_{z}\right)$ was defined in Eq. (16). From these equations, we see that $\lambda\left(m_{x}\right)$ assumes the values $\pm \Lambda$ and $\lambda\left(m_{z}\right)$ the values \pm 1 . Then, solving the above equations leads to the results given in Table XI. (These results look different from those in Ref. 3 because here the Fourier transforms are defined relative to the actual positions, whereas in Ref. 3 they are defined relative to the origin of the unit cell.)

Now, since the crystal is centrosymmetric, we take symmetry with respect to spatial inversion $\mathcal{I}$ into account. As before, recall that $\mathcal{I}$ transports the spin to its spatially inverted position without changing the orientation of the spin (a pseudovector). The change of position is equivalent to changing the sign of the wave vector in the Fourier transform and this is accomplished by complex conjugation. Since the Mn ions sit at centers of inversion symmetry, one has, for the Mn sublattices,

TABLE IX. Positions of the magnetic ions in the Pbnm structure of $\mathrm{TbMnO}_{3}$, with $x=0.9836$ and $y=0.0810$ (Ref. 47).

\begin{tabular}{llc}
\hline \hline $\mathrm{Mn}$ & $(1)=\left(0, \frac{1}{2}, 0\right)$ & $(2)=\left(\frac{1}{2}, 0,0\right)$ \\
& $(3)=\left(0, \frac{1}{2}, \frac{1}{2}\right)$ & $(4)=\left(\frac{1}{2}, 0, \frac{1}{2}\right)$ \\
$\mathrm{Tb}$ & $(5)=\left(x, y, \frac{1}{4}\right)$ & $(6)=\left(x+\frac{1}{2}, \bar{y}+\frac{1}{2}, \frac{3}{4}\right)$ \\
& $(7)=\left(\bar{x}, \bar{y}, \frac{3}{4}\right)$ & $(8)=\left(\bar{x}+\frac{1}{2}, y+\frac{1}{2}, \frac{1}{4}\right)$ \\
\hline \hline
\end{tabular}

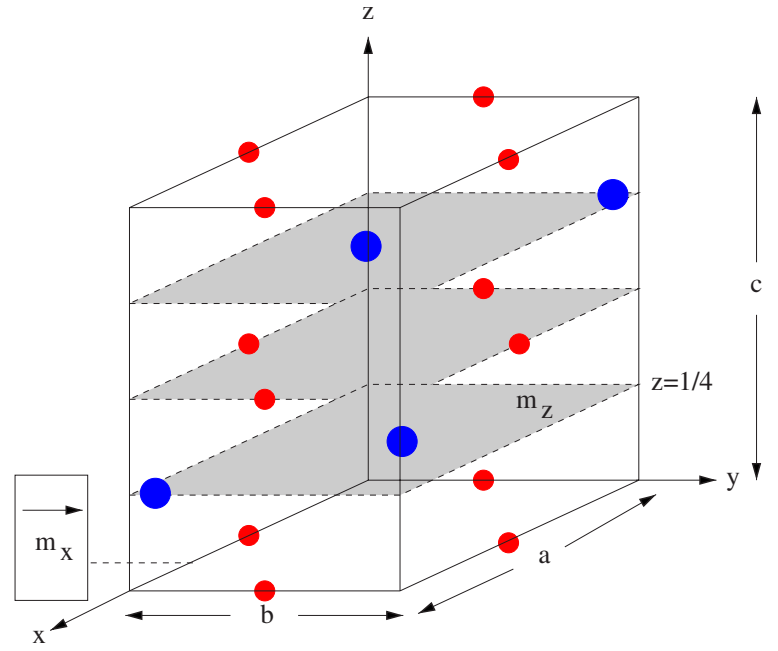

FIG. 4. (Color online) Mn sites (smaller circles, red online) and $\mathrm{Tb}$ sites (larger circles, blue online) in the primitive unit cell of $\mathrm{TbMnO}_{3}$. The $\mathrm{Tb}$ sites are in the shaded planes at $z=n \pm \frac{1}{4}$ and the Mn sites are in planes $z=n$ or $z=n+\frac{1}{2}$, where $n$ is an integer. The incommensurate wave vector is along the $\mathbf{b}$ axis. The mirror plane at $z=1 / 4$ is indicated and the glide plane $m_{x}$ is indicated by the mirror plane at $x=3 / 4$ followed by a translation (indicated by the arrow) of $b / 2$ along the $y$ axis.

$$
\mathcal{I} \mathbf{S}(\mathbf{q}, n)=\mathbf{S}(\mathbf{q}, n)^{*},
$$

where the second argument specifies the sublattice, as in Table IX. In order to discuss the symmetry of the coordinates, we define $x_{1}=n_{M}^{a}, x_{2}=n_{M}^{b}, x_{3}=n_{M}^{c}$ and for irreps $\Gamma_{1}$ and $\Gamma_{3}, x_{4}=n_{T 1}^{c}$ and $x_{5}=n_{T 2}^{c}$, whereas for irreps $\Gamma_{2}$ and $\Gamma_{4}, x_{4}$ $=n_{T 1}^{a}, x_{5}=n_{T 2}^{a}, x_{6}=n_{T 1}^{b}$, and $x_{7}=n_{T 2}^{b}$. Thus, Eq. (78) gives

$$
\mathcal{I} x_{n}=x_{n}^{*}, \quad n=1,2,3 \text {. }
$$

For the $\mathrm{Tb}$ ions, $\mathcal{I}$ interchanges sublattices 5 and 7 and interchanges sublattices 6 and 8 . So, we have

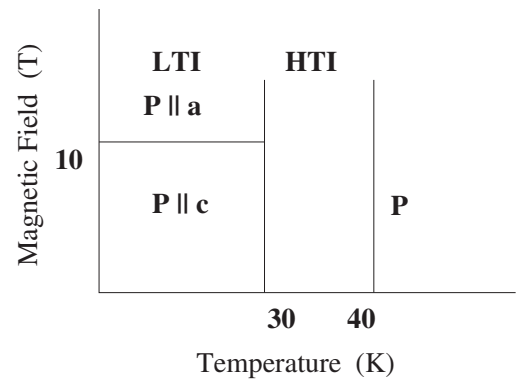

FIG. 5. Schematic phase diagram for TMO for magnetic fields up to $14 \mathrm{~T}$ applied along the a direction, taken from Ref. 48. Here, $\mathrm{P}$ is the paramagnetic phase, HTI is the "high-temperature" incommensurate phase in which (Ref. 3) the moments are essentially aligned along the $\mathbf{b}$ axis with a sinusoidally modulated amplitude according to irrep $\Gamma_{3}$, and LTI is the "low-temperature" incommensurate phase in which (Ref. 3) transverse order along the $\mathbf{c}$ axis appears to make an elliptically polarized order-parameter wave according to irreps $\Gamma_{3}$ and $\Gamma_{2}$. A spontaneous polarization $\mathbf{P}$ appears only in the LTI phase with $\mathbf{P}$ along the $\mathbf{c}$ axis for low magnetic field (Ref. 3). 
TABLE X. Transformation table for sublattice indices of TMO under various operations.

\begin{tabular}{lccc}
\hline \hline$\tau_{i}$ & $\tau_{f}\left(m_{x}\right)$ & $\tau_{f}\left(m_{z}\right)$ & $\tau_{f}(\mathcal{I})$ \\
\hline 1 & 2 & 3 & 1 \\
2 & 1 & 4 & 2 \\
3 & 4 & 1 & 3 \\
4 & 3 & 2 & 4 \\
5 & 8 & 5 & 7 \\
6 & 7 & 6 & 8 \\
7 & 6 & 7 & 5 \\
8 & 5 & 8 & 6 \\
\hline \hline
\end{tabular}

$$
\mathcal{I} \mathbf{S}(\mathbf{q}, 5)=\mathbf{S}(\mathbf{q}, 7)^{*}
$$

$$
\mathcal{I} \mathbf{S}(\mathbf{q}, 6)=\mathbf{S}(\mathbf{q}, 8)^{*}
$$

Therefore, we have

$$
\mathcal{I} x_{4}=x_{5}^{*}, \quad \mathcal{I} x_{6}=x_{7}^{*} .
$$

Now, we use the invariance of the free energy under $\mathcal{I}$ to write

$$
\begin{aligned}
F_{2} & =\sum_{X, \alpha ; Y, \beta} S_{\alpha}(\mathbf{q}, X)^{*} F_{n m} S_{\beta}(\mathbf{q}, Y)=\sum_{m, n} x_{n}^{*} G_{n m} x_{m} \\
& =\sum_{m, n}\left[\mathcal{I} x_{n}^{*}\right] G_{n m}\left[\mathcal{I} x_{m}\right],
\end{aligned}
$$

where the matrix $\mathbf{G}$ is Hermitian and we have implicitly limited consideration to whichever irrep is active.

For irreps $\Gamma_{1}$ and $\Gamma_{3}$, the matrix $\mathbf{G}$ in Eq. (82) couples five variables, $x_{1}, \ldots, x_{5}$. Equation (79) implies that the upper left $3 \times 3$ submatrix of $\mathbf{G}$ (which involves the variables $\left.x_{1}, \ldots, x_{3}\right)$ is real. Equations (79) and (81) imply that $G_{n, 4}$ $=G_{5, n}$ for $n=1,2,3$. We thus find that $\mathbf{G}$ assumes the form

$$
\mathbf{G}=\left[\begin{array}{lllll}
a & b & c & \alpha & \alpha^{*} \\
b & d & e & \beta & \beta^{*} \\
c & e & f & \gamma & \gamma^{*} \\
\alpha^{*} & \beta^{*} & \gamma^{*} & g & \delta \\
\alpha & \beta & \gamma & \delta^{*} & g
\end{array}\right],
$$

where the Roman letters are real valued and the Greek are complex valued. As shown in Appendix A, the form of this matrix ensures that the critical eigenvector can be taken to be of the form

$$
\psi=\left(n_{M}^{a}, n_{M}^{b}, n_{M}^{c}, n_{T 1}^{c}, n_{T 1}^{c *}\right) \equiv\left(r, s, t ; \rho, \rho^{*}\right),
$$

TABLE XI. Spin functions (i.e., actual position Fourier coeffi-

\begin{tabular}{|c|c|c|c|c|}
\hline Irrep & $\Gamma_{1}$ & $\Gamma_{2}$ & $\Gamma_{3}$ & $\Gamma_{4}$ \\
\hline$\lambda\left(m_{x}\right)$ & $+\Lambda$ & $-\Lambda$ & $-\Lambda$ & $+\Lambda$ \\
\hline$\lambda\left(m_{z}\right)$ & +1 & -1 & +1 & -1 \\
\hline $\mathbf{S}(\mathbf{q}, M 1)$ & $\begin{array}{c}n_{M}^{a} \\
-n_{M}^{b} \\
-n_{M}^{c}\end{array}$ & $\begin{array}{c}-n_{M}^{a} \\
n_{M}^{b} \\
n_{M}^{c}\end{array}$ & $\begin{array}{c}-n_{M}^{a} \\
n_{M}^{b} \\
n_{M}^{c}\end{array}$ & $\begin{array}{c}n_{M}^{a} \\
-n_{M}^{b} \\
-n_{M}^{c}\end{array}$ \\
\hline $\mathbf{S}(\mathbf{q}, M 2)$ & $\begin{array}{l}n_{M}^{a} \\
n_{M}^{b} \\
n_{M}^{c}\end{array}$ & $\begin{array}{c}n_{M}^{a} \\
n_{M}^{b} \\
n_{M}^{c}\end{array}$ & $\begin{array}{l}n_{M}^{a} \\
n_{M}^{b} \\
n_{M}^{c}\end{array}$ & $\begin{array}{l}n_{M}^{a} \\
n_{M}^{b} \\
n_{M}^{c}\end{array}$ \\
\hline $\mathbf{S}(\mathbf{q}, M 3)$ & $\begin{array}{c}-n_{M}^{a} \\
n_{M}^{b} \\
-n_{s}^{c}\end{array}$ & $\begin{array}{c}-n_{M}^{a} \\
n_{M}^{b} \\
-n_{M}^{c}\end{array}$ & $\begin{array}{c}n_{M}^{a} \\
-n_{M}^{b} \\
n_{M}^{c}\end{array}$ & $\begin{array}{c}n_{M}^{a} \\
-n_{M}^{b} \\
n_{M}^{c}\end{array}$ \\
\hline $\mathbf{S}(\mathbf{q}, M 4)$ & $\begin{array}{c}-n_{M}^{a} \\
-n_{M}^{b} \\
n_{M}^{c}\end{array}$ & $\begin{array}{c}n_{M}^{a} \\
n_{M}^{b} \\
-n_{M}^{c}\end{array}$ & $\begin{array}{c}-n_{M}^{a} \\
-n_{M}^{b} \\
n_{M}^{c}\end{array}$ & $\begin{array}{c}n_{M}^{a} \\
n_{M}^{b} \\
-n_{M}^{c}\end{array}$ \\
\hline $\mathbf{S}(\mathbf{q}, T 1)$ & $\begin{array}{c}0 \\
0 \\
n_{T 1}^{c}\end{array}$ & $\begin{array}{c}n_{T 1}^{a} \\
n_{T 1}^{b} \\
0\end{array}$ & $\begin{array}{c}0 \\
0 \\
n_{T 1}^{c}\end{array}$ & $\begin{array}{c}n_{T 1}^{a} \\
n_{T 1}^{b} \\
0\end{array}$ \\
\hline $\mathbf{S}(\mathbf{q}, T 2)$ & $\begin{array}{c}0 \\
0 \\
-n_{T 2}^{c}\end{array}$ & $\begin{array}{c}-n_{T 2}^{c} \\
n_{T 2}^{b} \\
0\end{array}$ & $\begin{array}{c}0 \\
0 \\
n_{T 2}^{c}\end{array}$ & $\begin{array}{c}n_{T 2}^{a} \\
-n_{T 2}^{b} \\
0\end{array}$ \\
\hline $\mathbf{S}(\mathbf{q}, T 3)$ & $\begin{array}{c}0 \\
0 \\
n_{T 2}^{c}\end{array}$ & $\begin{array}{c}n_{T 2}^{a} \\
n_{T 2}^{b} \\
0\end{array}$ & $\begin{array}{c}0 \\
0 \\
n_{T 2}^{c}\end{array}$ & $\begin{array}{c}n_{T 2}^{a} \\
n_{T 2}^{b} \\
0\end{array}$ \\
\hline $\mathbf{S}(\mathbf{q}, T 4)$ & $\begin{array}{c}0 \\
0 \\
-n_{T 1}^{c}\end{array}$ & $\begin{array}{c}-n_{T 1}^{a} \\
n_{T 1}^{b} \\
0\end{array}$ & $\begin{array}{c}0 \\
0 \\
n_{T 1}^{c}\end{array}$ & $\begin{array}{c}n_{T 1}^{a} \\
-n_{T 1}^{b} \\
0\end{array}$ \\
\hline
\end{tabular}
cients) within the unit cell of TMO for wave vector $(0, q, 0)$ which are eigenvectors of $m_{x}$ and $m_{z}$ with the eigenvalues listed, with $\Lambda$ $=\exp (i \pi q)$. All the parameters are complex valued. The irreducible representation (irrep) is labeled as in Ref. 3. Inversion symmetry is not yet taken into account. Note that the two Tb orbits, (T1-T4) and (T2-T3), have independent complex amplitudes.

where the Roman letters are real and the Greek ones complex. Of course, because the vector can be complex, we should include an overall phase factor (which amounts to arbitrarily placing the origin of the incommensurate structure), so that more generally

$$
\psi=e^{i \phi}\left(r, s, t ; \rho, \rho^{*}\right) .
$$

For irreps $\Gamma_{2}$ and $\Gamma_{4}$, the matrix $\mathbf{G}$ in Eq. (82) couples the seven variables, $x_{1}, \ldots, x_{7}$, listed just above Eq. (79). Equations (79) and (81) imply that $G_{n, 4}=G_{5, n}$ and $G_{n, 6}=G_{7, n}$ for $n=1,2,3$. Also, Eq. (81) implies similar relations within the lower right $4 \times 4$ submatrix involving the variables $x_{4}, \ldots, x_{7}$. Therefore, $\mathbf{G}$ assumes the form 
TABLE XII. The same as Table XI except that a part from an overall phase $\phi_{\Gamma}$ for each irrep, inversion symmetry restricts all the manganese Fourier coefficients to be real and all the $\mathrm{Tb}$ coefficients to have the indicated phase relations.

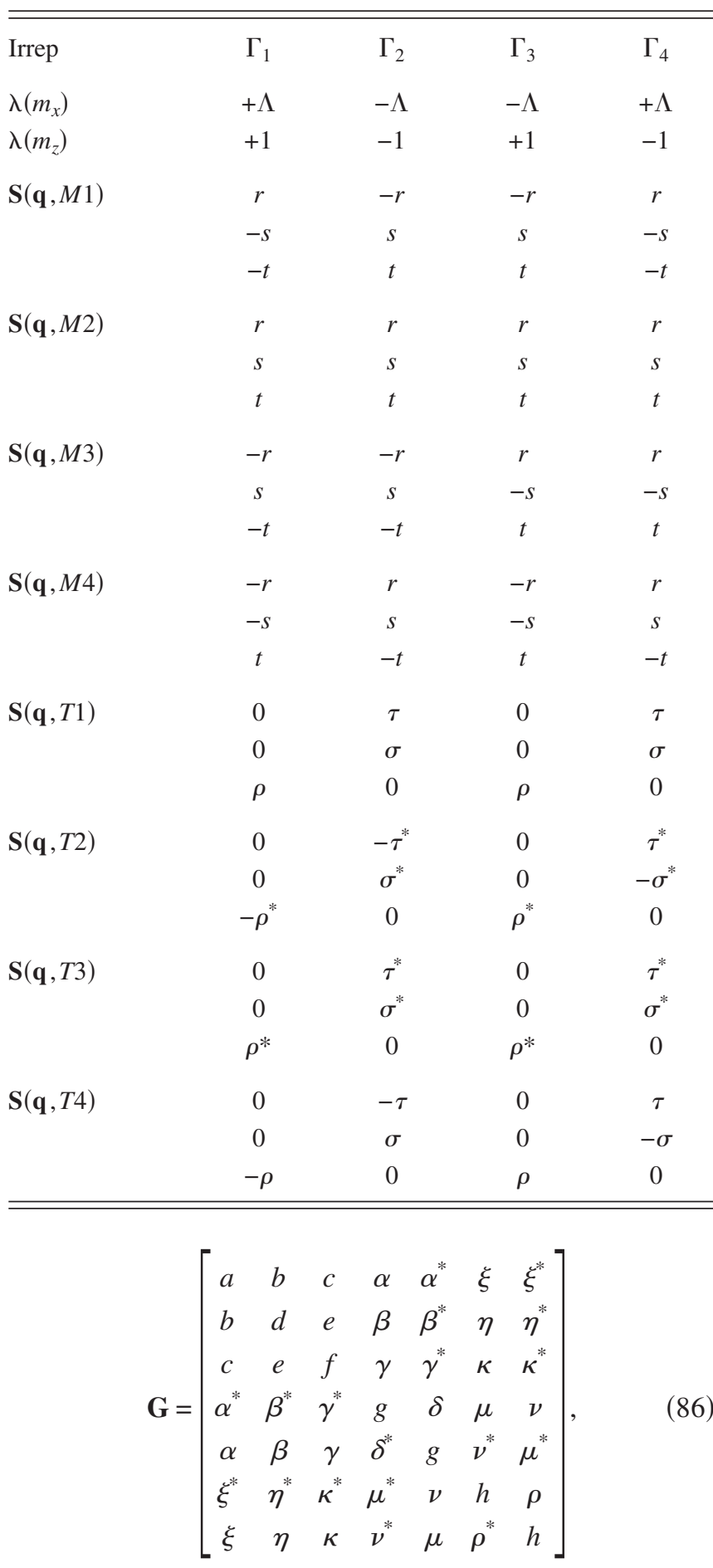

where Roman letters are real and Greek are complex. As shown in appendix A, this form ensures that the eigenvectors are of the form

$\psi=\left(n_{M}^{a}, n_{M}^{b}, n_{M}^{c}, n_{T 1}^{a}, n_{T 2}^{a}, m_{T 1}^{b}, n_{T 2}^{b}\right)=e^{i \phi}\left(r, s, t ; \tau, \tau^{*}, \sigma, \sigma^{*}\right)$.

These results are summarized in Table XII. Note that the use of inversion symmetry fixes most of the phases and relates the amplitudes of the two $\mathrm{Tb}$ orbits, thereby eliminating almost half the fitting parameters. ${ }^{3}$

\section{Order parameters}

We now introduce order parameters $\boldsymbol{\sigma}_{n}(\mathbf{q}) \equiv \sigma_{n} e^{-i \phi_{n}}$ for irrep $\Gamma_{n}$ in terms of which we can write the spin distribution. For instance, under $\Gamma_{3}$ one has

$$
\begin{gathered}
S_{x}(\mathbf{r}, M 1)=-2 r \sigma_{3} \cos \left(q y+\phi_{3}\right), \\
S_{y}(\mathbf{r}, M 1)=2 s \sigma_{3} \cos \left(q y+\phi_{3}\right), \\
S_{z}(\mathbf{r}, M 1)=2 t \sigma_{3} \cos \left(q y+\phi_{3}\right), \\
S_{x}(\mathbf{r}, M 2)=2 r \sigma_{3} \cos \left(q y+\phi_{3}\right), \\
S_{y}(\mathbf{r}, M 2)=2 s \sigma_{3} \cos \left(q y+\phi_{3}\right), \\
S_{z}(\mathbf{r}, M 2)=2 t \sigma_{3} \cos \left(q y+\phi_{3}\right), \\
S_{x}(\mathbf{r}, T 1)=S_{y}(\mathbf{r}, T 1)=0, \\
S_{z}(\mathbf{r}, T 1)=2 \rho \sigma_{3} \cos \left(q y+\phi_{3}+\phi_{\rho}\right), \\
S_{x}(\mathbf{r}, T 2)=S_{y}(\mathbf{r}, T 2)=0, \\
S_{z}(\mathbf{r}, T 2)=2 \rho \sigma_{3} \cos \left(q y+\phi_{3}-\phi_{\rho}\right),
\end{gathered}
$$

where we set $\boldsymbol{\rho}=\rho e^{i \phi_{\rho}}$ and the parameters are normalized by

$$
r^{2}+s^{2}+t^{2}+\rho^{2}=1 .
$$

In Eq. $(88), \mathbf{r} \equiv(x, y, z)$ is the actual position of the spin in question. From Table XI, one can obtain the symmetry properties of the order parameters for each irrep. For instance,

$$
\begin{array}{ll}
m_{x} \boldsymbol{\sigma}_{1}(\mathbf{q})=+\Lambda \boldsymbol{\sigma}_{1}(\mathbf{q}), & m_{z} \boldsymbol{\sigma}_{1}(\mathbf{q})=+\boldsymbol{\sigma}_{1}(\mathbf{q}), \\
m_{x} \boldsymbol{\sigma}_{2}(\mathbf{q})=-\Lambda \boldsymbol{\sigma}_{2}(\mathbf{q}), & m_{z} \boldsymbol{\sigma}_{2}(\mathbf{q})=-\boldsymbol{\sigma}_{2}(\mathbf{q}), \\
m_{x} \boldsymbol{\sigma}_{3}(\mathbf{q})=-\Lambda \boldsymbol{\sigma}_{3}(\mathbf{q}), & m_{z} \boldsymbol{\sigma}_{3}(\mathbf{q})=+\boldsymbol{\sigma}_{3}(\mathbf{q}) \\
m_{x} \boldsymbol{\sigma}_{4}(\mathbf{q})=+\Lambda \boldsymbol{\sigma}_{4}(\mathbf{q}), & m_{z} \boldsymbol{\sigma}_{4}(\mathbf{q})=-\boldsymbol{\sigma}_{4}(\mathbf{q}),
\end{array}
$$

and

$$
\mathcal{I} \boldsymbol{\sigma}_{n}(\mathbf{q})=\boldsymbol{\sigma}_{n}^{*}(\mathbf{q})
$$

Note that in contrast to the case of NVO, inversion symmetry does not fix all the phases. However, it again drastically reduces the number of possible magnetic structure parameters which have to be determined. In particular, it is only by using inversion that one finds that the magnitudes of the Fourier coefficients of the two distinct $\mathrm{Tb}$ sites have to be the same. Note that if we choose the origin so that $\phi=0$ (which amounts to renaming the origin so that that becomes true), then we recover inversion symmetry (taking account that inversion interchanges terbium sublattices 3 and 1). One can determine that the spin structure is inversion invariant when one condenses a single representation. 
TABLE XIII. The same as Table VIII. General positions for Pbam.

\begin{tabular}{ll}
\hline \hline$E \mathbf{r}=(x, y, z)$ & $2_{x} \mathbf{r}=\left(x+\frac{1}{2}, \bar{y}+\frac{1}{2}, \bar{z}\right)$ \\
$2_{z} \mathbf{r}=(\bar{x}, \bar{y}, z)$ & $2_{y} \mathbf{r}=\left(\bar{x}+\frac{1}{2}, y+\frac{1}{2}, \bar{z}\right)$ \\
$\mathcal{I} \mathbf{r}=(\bar{x}, \bar{y}, \bar{z})$ & $m_{x} \mathbf{r}=\left(\bar{x}+\frac{1}{2}, y+\frac{1}{2}, z\right)$ \\
$m_{z} \mathbf{r}=(x, y, \bar{z})$ & $m_{y} \mathbf{r}=\left(x+\frac{1}{2}, \bar{y}+\frac{1}{2}, z\right)$ \\
\hline \hline
\end{tabular}

The experimentally determined structure of the hightemperature incommensurate (HTI) and low-temperature incommensurate (LTI) phases is described in the caption of Fig. 5 and numerical values of the structure parameters are given in Ref. 3.

The result of Table XII applies to other manganates provided their wave vector is also of the form $\left(0, q_{y}, 0\right)$. This includes $\mathrm{DMO},{ }^{9} \mathrm{YMnO}_{3},{ }^{50}$ and $\mathrm{HoMnO}_{3} .{ }^{51,52}$ Both these systems order into an incommensurate structure at about $T_{c}$ $\approx 42 \mathrm{~K}$. The $\mathrm{Y}$ compound has a second lower-temperature incommensurate phase, whereas the Ho compound has a lower-temperature commensurate phase.

\section{C. $\mathrm{TbMn}_{2} \mathrm{O}_{5}$}

The space group of $\mathrm{TbMn}_{2} \mathrm{O}_{5}$ (TMO25) is Pbam (No. 55 in Ref. 33) and its general positions are listed in Table XIII. The positions of the magnetic ions are given in Table XIV and are shown in Fig. 6.

We will address the situation just below the ordering temperature of $43 \mathrm{~K} .{ }^{55}$ We take the ordering wave vector to be ${ }^{55}$ to be $\left(\frac{1}{2}, 0, q\right)$ with $q \approx 0.306$. (This may be an approximate value. ${ }^{56}$ ) [The following calculation involves a great deal of algebra which may be skipped. The explicit result for the spin structure is given in Eq. (123).] Initially, we assume that the possible spin configurations consistent with a continuous transition at such a wave vector are eigenvectors of the operators $m_{x}$ and $m_{y}$ which leave the wave vector invariant. We proceed as for TMO. We use the unit cell Fourier transforms and write the eigenvector conditions for transformation by $m_{x}$ as

$$
S_{\alpha}\left(\mathbf{q}, \tau_{f}\right)^{\prime}=\xi_{\alpha}\left(m_{x}\right) S_{\alpha}\left(\mathbf{q}, \tau_{i}\right) e^{i \mathbf{q}\left(\mathbf{R}_{f}-\mathbf{R}_{i}\right)}=\lambda_{x} S_{\alpha}\left(\mathbf{q}, \tau_{f}\right),
$$

where $\tau_{i}$ and $\mathbf{R}_{i}$ are, respectively, the sublattice indices and unit cell locations before transformation and $\tau_{f}$ and $\mathbf{R}_{f}$ are

TABLE XIV. Positions of the magnetic ions of $\mathrm{TbMn}_{2} \mathrm{O}_{5}$ in the Pbam structure. Here, $x=0.09, y=-0.15, z=0.25$ (Ref. 53), $X$ $=0.14$, and $Y=0.17$ (Ref. 54). All these values are taken from the isostructural compound $\mathrm{HoMn}_{2} \mathrm{O}_{5}$.

\begin{tabular}{lrr}
\hline \hline $\mathrm{Mn}^{3+}$ & $(1)=(x, y, 0)$ & $(2)=(\bar{x}, \bar{y}, 0)$ \\
& $(3)=\left(\bar{x}+\frac{1}{2}, y+\frac{1}{2}, 0\right)$ & $(4)=\left(x+\frac{1}{2}, \bar{y}+\frac{1}{2}, 0\right)$ \\
$\mathrm{Mn}^{4+}$ & $(5)=\left(\frac{1}{2}, 0, z\right)$ & $(6)=\left(0, \frac{1}{2}, z\right)$ \\
& $(7)=\left(\frac{1}{2}, 0, \bar{z}\right)$ & $(8)=\left(0, \frac{1}{2}, \bar{z}\right)$ \\
$\mathrm{RE}$ & $(9)=\left(X, Y, \frac{1}{2}\right)$ & $(10)=\left(\bar{X}, \bar{Y}, \frac{1}{2}\right)$ \\
& $(11)=\left(\bar{X}+\frac{1}{2}, Y+\frac{1}{2}, \frac{1}{2}\right)$ & $(12)=\left(X+\frac{1}{2}, \bar{Y}+\frac{1}{2}, \frac{1}{2}\right)$ \\
\hline
\end{tabular}
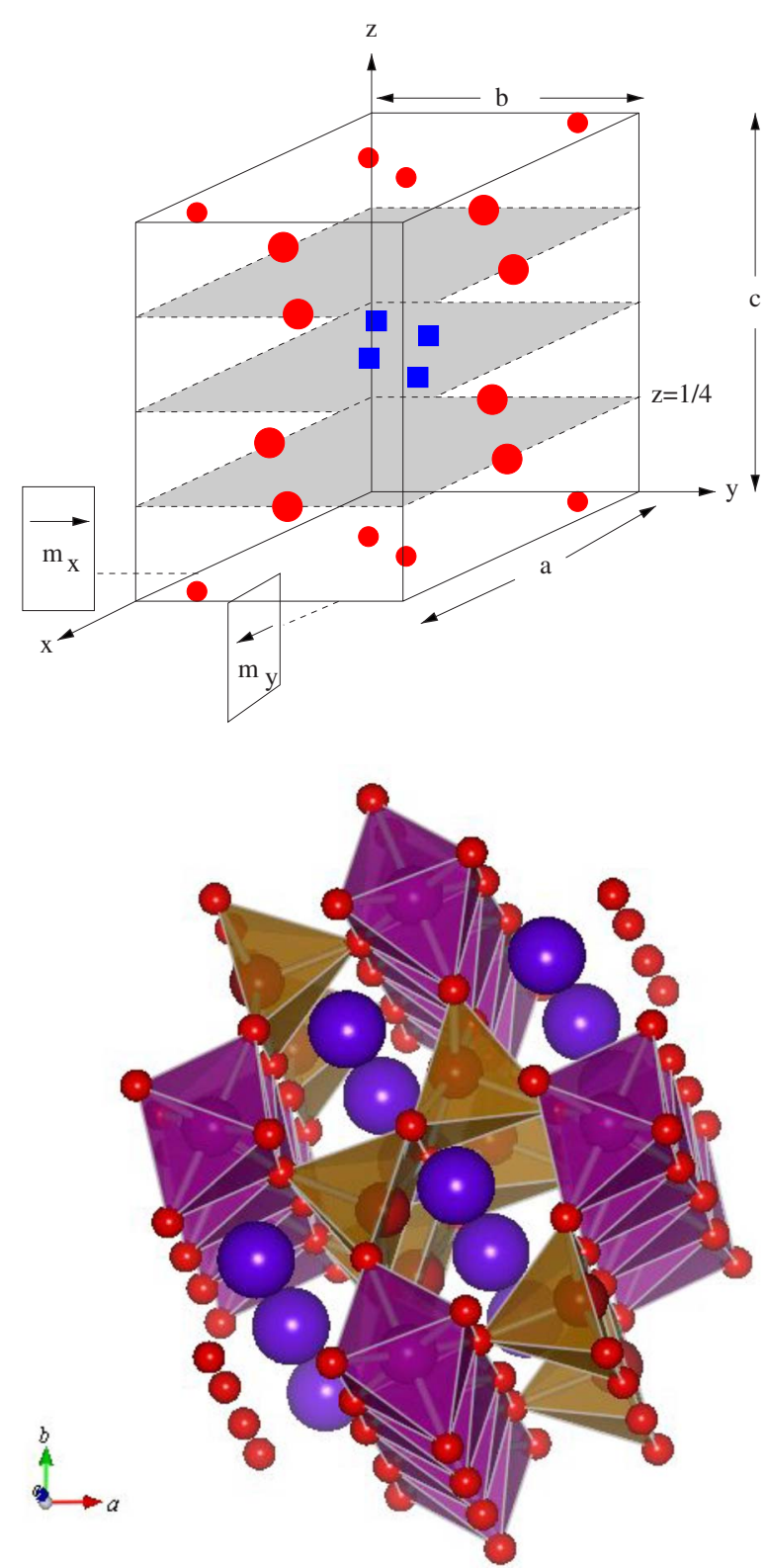

FIG. 6. (Color online) Two representations of $\mathrm{TbMn}_{2} \mathrm{O}_{5}$. Top: Mn sites (red online) with smaller circles $\left(\mathrm{Mn}^{3+}\right)$ and larger circles $\left(\mathrm{Mn}^{4+}\right)$ and $\mathrm{Tb}$ sites (squares, blue online blue) in the primitive unit cell of $\mathrm{TbMn}_{2} \mathrm{O}_{5}$. The $\mathrm{Mn}^{+4}$ sites are in the shaded planes at $z$ $=n \pm \delta$ with $\delta \approx 0.25$ and the $\mathrm{Mn}^{+3}$ sites are in planes $z=n$, where $n$ is an integer. The $\mathrm{Tb}$ ions are in the planes $z=n+\frac{1}{2}$. The glide plane $m_{x}$ is indicated by the mirror plane at $x=3 / 4$ followed by a translation (indicated by the arrow) of $b / 2$ along the $y$ axis and similarly for the glide plane $m_{y}$. Bottom: Perspective view. Here, the $\mathrm{Mn}^{3+}$ are inside oxygen pyramids of small balls and the $\mathrm{Mn}^{4+}$ are inside oxygen octahedra.

those after transformation. The eigenvalue equation for transformation by $m_{y}$ is

$$
S_{\alpha}\left(\mathbf{q}, \tau_{f}\right)^{\prime}=\xi_{\alpha}\left(m_{y}\right) S_{\alpha}\left(\mathbf{q}, \tau_{i}\right) e^{i \mathbf{q}\left(\mathbf{R}_{f}-\mathbf{R}_{i}\right)}=\lambda_{y} S_{\alpha}\left(\mathbf{q}, \tau_{f}\right) .
$$

If one attempts to construct spin functions which are simultaneously eigenfunctions of $m_{x}$ and $m_{y}$, one finds that these equations yield no solution. While it is, of course, true 
that the operations $m_{x}$ and $m_{y}$ take an eigenfunction into an eigenfunction, it is only for irreps of dimension 1 that the initial and final eigenfunctions are the same, as we have assumed. The present case, when the wave vector is at the edge of the Brillouin zone, is analogous to the phenomenon of "sticking" where, for nonsymmorphic space group (i.e., those having a screw axis or a glide plane), the energy bands (or phonon spectra) have an almost mysterious degeneracy at the zone boundary ${ }^{57}$ and the only active irrep has dimension 2 . This means that the symmetry operations induce transformations within the subspace of pairs of eigenfunctions. We now determine such pairs of eigenfunctions by a straightforward approach which does not require any knowledge of group theory. Here, we explicitly consider the symmetries of the matrix $\chi^{-1}$ for the quadratic terms in the free energy which here is a $36 \times 36$ dimensional matrix, which we write as

$$
\boldsymbol{\chi}^{-1}=\left[\begin{array}{ccc}
\mathbf{M}^{(x x)} & \mathbf{M}^{(x y)} & \mathbf{M}^{(x z)} \\
\mathbf{M}^{(x y)^{\dagger}} & \mathbf{M}^{(y y)} & \mathbf{M}^{(y z)} \\
\mathbf{M}^{(z x)^{\dagger}} & \mathbf{M}^{(y z)^{\dagger}} & \mathbf{M}^{(z z)}
\end{array}\right],
$$

where $\mathbf{M}^{(a b)}$ is a 12 dimensional submatrix which describes coupling between $a$-component and $b$-component spins and is indexed by sublattice indices $\tau$ and $\tau^{\prime}$. The symmetries we invoke are operations of the glide planes $m_{x}$ and $m_{y}$, which conserve wave vector (to within a reciprocal lattice vector), and $\mathcal{I}$, whose effect is usually ignored. To guide the reader through the ensuing calculation, we summarize the main steps. We first analyze separately the sectors involving the $x$, $y$, and $z$ spin components. We develop a unitary transformation which takes $\mathbf{M}^{(\alpha \alpha)}$ into a matrix all of whose elements are real. This fixes the phases within the 12 dimensional space of the $\alpha$ spin components within the unit cell (assuming that these relations are not invalidated by the form of $\mathbf{M}^{(\alpha \beta)}$, with $\left.\alpha \neq \beta\right)$. The relative phases between different spin components are fixed by showing that the unitary transformation introduced above leads to $\mathbf{M}^{(x y)}$ having all realvalued matrix elements and $\mathbf{M}^{(x z)}$ and $\mathbf{M}^{(y z)}$ having all purely imaginary matrix elements. The conclusion, then, is that the phases in the sectors of $x$ and $y$ components are coupled in phase and the sector of $z$ components are out of phase with the $x$ and $y$ components.

\section{1. $x$ components}

As a preliminary, in Table XV we list the effect of the symmetry operations on the sublattice index. When these symmetries are used, one finds that the $12 \times 12$ submatrix $\mathbf{M}^{(x x)}$ which couples only the $x$ components of spins assumes the form

$\left[\begin{array}{cccc|cc|cc|cccc}A & g & h & 0 & \alpha & \beta & \alpha^{*} & \beta^{*} & a & b & c & d \\ g & A & 0 & -h & -\alpha & \beta & -\alpha^{*} & \beta^{*} & b & a & -d & -c \\ h & 0 & A & g & \beta & \alpha & \beta^{*} & \alpha^{*} & c & d & a & b \\ 0 & -h & g & A & \beta & -\alpha & \beta^{*} & -\alpha^{*} & -d & -c & b & a \\ \hline \alpha^{*} & -\alpha^{*} & \beta^{*} & \beta^{*} & B & 0 & \epsilon & 0 & \gamma & -\gamma & \delta & \delta \\ \beta^{*} & \beta^{*} & \alpha^{*} & -\alpha^{*} & 0 & B & 0 & \epsilon & \delta & \delta & \gamma & -\gamma \\ \hline \alpha & -\alpha & \beta & \beta & \epsilon^{*} & 0 & B & 0 & \gamma^{*} & -\gamma^{*} & \delta^{*} & \delta^{*} \\ \beta & \beta & \alpha & -\alpha & 0 & \epsilon^{*} & 0 & B & \delta^{*} & \delta^{*} & \gamma^{*} & -\gamma^{*} \\ \hline a & b & c & -d & \gamma^{*} & \delta^{*} & \gamma & \delta & C & e & f & 0 \\ b & a & d & -c & -\gamma^{*} & \delta^{*} & -\gamma & \delta & e & C & 0 & -f \\ c & -d & a & b & \delta^{*} & \gamma^{*} & \delta & \gamma & f & 0 & C & e \\ d & -c & b & a & \delta^{*} & -\gamma^{*} & \delta & -\gamma & 0 & -f & e & C\end{array}\right]$,

where Roman letters are real quantities and Greek ones complex. (In this matrix, the lines are used to separate different Wyckoff orbits.) The numbering of the rows and columns follows from Table XIV. I give a few examples of how symmetry is used to get this form. Consider the term $T_{1}$, where

$$
T_{1}=\chi_{1,5}^{-1} S_{x}(-\mathbf{q}, 1) S_{x}(\mathbf{q}, 5)
$$

Using Table XV, we transform this by $m_{x}$ into

$$
T_{1}^{\prime}=\chi_{1,5}^{-1} S_{x}(-\mathbf{q}, 3) S_{x}(\mathbf{q}, 6),
$$

which says that the 1,5 matrix element is equal to the 3,6 matrix element. (Note that in writing down $T_{1}^{\prime}$, we did not need to worry about $\xi_{\alpha}$, since this factor comes in squared as unity.) Likewise, if we transform by $m_{y}$, we get

$$
T_{1}^{\prime}=\chi_{15}^{-1}\left[S_{x}(-\mathbf{q}, 4)\right]\left[-S_{x}(\mathbf{q}, 6)\right],
$$

which says that the 1,5 matrix element is equal to the negative of the 4,6 matrix element. If we transform by $m_{x} m_{y}$, we get

$$
T_{1}^{\prime}=\chi_{1,5}^{-1}\left[S_{x}(-\mathbf{q}, 2)\right]\left[-S_{x}(\mathbf{q}, 5)\right],
$$


which says that the 1,5 matrix element is equal to the negative of the 2,5 matrix element. To illustrate the effect of $\mathcal{I}$ on $T_{1}$ we write

$$
T_{1}^{\prime}=\chi_{1,5}^{-1}\left[S_{x}(\mathbf{q}, 2)\right]\left[-S_{x}(-\mathbf{q}, 7)\right]
$$

so that the 1,5 element is the negative of the 7,2 element. From the form of the matrix in Eq. (95) (or equivalently referring to Table XXIII in Appendix B), we see that we bring this matrix into block diagonal form by introducing the wave functions for $S_{x}(\mathbf{q}, \tau)$,

$\left\|\begin{array}{ccccccccccccc}\tau= & 1 & 2 & 3 & 4 & 5 & 6 & 7 & 8 & 9 & 10 & 11 & 12 \\ \sqrt{2} O_{1, \tau}^{(x, 1)}= & 1 & 0 & 1 & 0 & 0 & 0 & 0 & 0 & 0 & 0 & 0 & 0 \\ \sqrt{2} O_{2, \tau}^{(x, 1)}= & 0 & 1 & 0 & 1 & 0 & 0 & 0 & 0 & 0 & 0 & 0 & 0 \\ 2 O_{3, \tau}^{(x, 1)}= & 0 & 0 & 0 & 0 & 1 & 1 & 1 & 1 & 0 & 0 & 0 & 0 \\ 2 O_{4, \tau}^{(x, 1)}= & 0 & 0 & 0 & 0 & i & i & -i & -i & 0 & 0 & 0 & 0 \\ \sqrt{2} O_{5, \tau}^{(x, 1)}= & 0 & 0 & 0 & 0 & 0 & 0 & 0 & 0 & 1 & 0 & 1 & 0 \\ \sqrt{2} O_{6, \tau}^{(x, 1)}= & 0 & 0 & 0 & 0 & 0 & 0 & 0 & 0 & 0 & 1 & 0 & 1\end{array}\right\|$.

The superscripts $\alpha$ and $n$ on $\mathbf{O}$ label, respectively, the Cartesian component and the column of the irrep according to which the wave function transforms. The subscripts $m$ and $\tau$ label, respectively, the index number of the wave function and the sublattice label. Let $\mathbf{O}_{p}^{\alpha, n}$ be a vector with components $O_{p, 1}^{\alpha, n}, O_{p, 2}^{\alpha, n} \ldots, O_{p, 12}^{\alpha, n}$. Then, $\left\langle\mathbf{O}_{n}^{(x, 1)}\left|M^{(x x)}\right| \mathbf{O}_{m}^{(x, 1)}\right\rangle \equiv\left\langle n\left|M^{(x x)}\right| m\right\rangle$ is

$$
\left[\begin{array}{cccccc}
A+h & g & \alpha^{\prime}+\beta^{\prime} & -\alpha^{\prime \prime}-\beta^{\prime \prime} & a+c & b+d \\
g & A-h & \beta^{\prime}-\alpha^{\prime} & \alpha^{\prime \prime}-\beta^{\prime \prime} & b-d & a-c \\
\alpha^{\prime}+\beta^{\prime} & \beta^{\prime}-\alpha^{\prime} & B+\epsilon^{\prime} & \epsilon^{\prime \prime} & \delta^{\prime}+\gamma^{\prime} & \delta^{\prime}-\gamma^{\prime} \\
-\alpha^{\prime \prime}-\beta^{\prime \prime} & \alpha^{\prime \prime}-\beta^{\prime \prime} & \epsilon^{\prime \prime} & B-\epsilon^{\prime} & \delta^{\prime \prime}+\gamma^{\prime \prime} & \delta^{\prime \prime}-\gamma^{\prime \prime} \\
a+c & b-d & \delta^{\prime}+\gamma^{\prime} & \delta^{\prime \prime}+\gamma^{\prime \prime} & C+f & e \\
b+d & a-c & \delta^{\prime}-\gamma^{\prime} & \delta^{\prime \prime}-\gamma^{\prime \prime} & e & C-f
\end{array}\right],
$$

where the coefficients are separated into real and imaginary parts as $\sqrt{2} \alpha=\alpha^{\prime}+i \alpha^{\prime \prime}, \sqrt{2} \beta=\beta^{\prime}+i \beta^{\prime \prime} \sqrt{2} \gamma=\gamma^{\prime}+i \gamma^{\prime}$, and $\sqrt{2} \delta=\delta^{\prime}+i \delta^{\prime \prime}$. There are no nonzero matrix elements between wave functions which transform according to different columns of the irrep.

The partners of these functions can be found from

$$
\mathbf{O}_{n}^{(x, 2)}=m_{y} \mathbf{O}_{n}^{(x, 1)},
$$

so that, using Table XV and including the factor $\xi_{\alpha}$, we get

$\| \begin{array}{ccccccccccccc}\tau= & 1 & 2 & 3 & 4 & 5 & 6 & 7 & 8 & 9 & 10 & 11 & 12 \\ \sqrt{2} O_{1, \tau}^{(x, 2)}= & 0 & 1 & 0 & -1 & 0 & 0 & 0 & 0 & 0 & 0 & 0 & 0 \\ \sqrt{2} O_{2, \tau}^{(x, 2)}= & 1 & 0 & -1 & 0 & 0 & 0 & 0 & 0 & 0 & 0 & 0 & 0 \\ 2 O_{3, \tau}^{(x, 2)}= & 0 & 0 & 0 & 0 & -1 & 1 & -1 & 1 & 0 & 0 & 0 & 0 \\ 2 O_{4, \tau}^{(x, 2)}= & 0 & 0 & 0 & 0 & -i & i & i & -i & 0 & 0 & 0 & 0 \\ \sqrt{2} O_{5, \tau}^{(x, 2)}= & 0 & 0 & 0 & 0 & 0 & 0 & 0 & 0 & 0 & 1 & 0 & -1 \\ \sqrt{2} O_{6, \tau}^{(x, 2)}= & 0 & 0 & 0 & 0 & 0 & 0 & 0 & 0 & 1 & 0 & -1 & 0\end{array}$

Within this subspace, the matrix $\left\langle n\left|M^{(x x)}\right| m\right\rangle$ is the same as in Eq. (102) because

$$
\left\langle n\left|m_{y}^{-1} M^{(x x)} m_{y}\right| m\right\rangle=\left\langle n\left|M^{(x x)}\right| m\right\rangle .
$$

These functions transform as expected for a twodimensional irrep, namely,

$$
\begin{gathered}
m_{x}\left[\begin{array}{l}
\mathbf{O}_{n}^{(x, 1)} \\
\mathbf{O}_{n}^{(x, 2)}
\end{array}\right]=\left[\begin{array}{c}
\mathbf{O}_{n}^{(x, 1)} \\
-\mathbf{O}_{n}^{(x, 2)}
\end{array}\right], \\
m_{y}\left[\begin{array}{l}
\mathbf{O}_{n}^{(x, 1)} \\
\mathbf{O}_{n}^{(x, 2)}
\end{array}\right]=\left[\begin{array}{c}
\mathbf{O}_{n}^{(x, 2)} \\
-\mathbf{O}_{n}^{(x, 1)}
\end{array}\right] .
\end{gathered}
$$


TABLE XV. Transformation table for sublattice indices with associated factors for TMO25 under various operations as defined by Eq. (20). For $m_{x}$, one has $\exp \left[i \mathbf{q} \cdot\left(\mathbf{R}_{f}-\mathbf{R}_{i}\right)\right]=1$ for all cases and for $m_{x} m_{y} \mathcal{I}$ the analogous factor is +1 in all cases and this operator relates $S_{\alpha}(\mathbf{q}, \tau)$ and $S_{\alpha}(\mathbf{q}, \tau)^{*}$. NOTE: This table does not include the factor of $\xi_{\alpha}(\mathcal{O})$ which may be associated with an operation.

\begin{tabular}{|c|c|c|c|c|c|c|c|c|}
\hline \multirow[b]{2}{*}{$n_{i}$} & \multirow{2}{*}{$\begin{array}{l}m_{x} \\
n_{f}\end{array}$} & \multicolumn{2}{|c|}{$m_{y}{ }^{\mathrm{a}}$} & \multicolumn{2}{|c|}{$m_{x} m_{y}{ }^{\mathrm{a}}$} & \multicolumn{2}{|c|}{$\mathcal{I}^{\mathrm{b}}$} & \multirow{2}{*}{$\begin{array}{c}m_{x} m_{y} \mathcal{I} \\
n_{f}\end{array}$} \\
\hline & & $n_{f}$ & $e^{i \phi}$ & $n_{f}$ & $e^{i \phi}$ & $n_{f}$ & $e^{i \phi^{\prime}}$ & \\
\hline 1 & 3 & 4 & 1 & 2 & 1 & 2 & 1 & 1 \\
\hline 2 & 4 & 3 & 1 & 1 & 1 & 1 & 1 & 2 \\
\hline 3 & 1 & 2 & -1 & 4 & -1 & 4 & -1 & 3 \\
\hline 4 & 2 & 1 & -1 & 3 & -1 & 3 & -1 & 4 \\
\hline 5 & 6 & 6 & -1 & 5 & -1 & 7 & -1 & 7 \\
\hline 6 & 5 & 5 & 1 & 6 & 1 & 8 & 1 & 8 \\
\hline 7 & 8 & 8 & -1 & 7 & -1 & 5 & -1 & 5 \\
\hline 8 & 7 & 7 & 1 & 8 & 1 & 6 & 1 & 6 \\
\hline 9 & 11 & 12 & 1 & 10 & 1 & 10 & 1 & 9 \\
\hline 10 & 12 & 11 & 1 & 9 & 1 & 9 & 1 & 10 \\
\hline 11 & 9 & 10 & -1 & 12 & -1 & 12 & -1 & 11 \\
\hline 12 & 10 & 9 & -1 & 11 & -1 & 11 & -1 & 12 \\
\hline
\end{tabular}

${ }^{\mathrm{a}} \boldsymbol{\phi}=\mathbf{q} \cdot\left(\mathbf{R}_{f}-\mathbf{R}_{i}\right)$, as required by Eq. (19).

${ }^{\mathrm{b}} \boldsymbol{\phi}^{\prime}=\mathbf{q} \cdot\left(\boldsymbol{\tau}_{i}+\boldsymbol{\tau}_{f}\right)$, as required by Eq. (22).

We will refer to the transformed coordinates of Eqs. (101) and (104) as "symmetry adapted coordinates." The fact that the model-specific matrix that couples them is real means that the critical eigenvector is a linear combination of symmetry adapted coordinates with real coefficients.

\section{2. y components}

The $12 \times 12$ matrix $M^{(y y)}$ coupling $y$ components of spin has exactly the same form as that given in Eq. (95), although the values of the constants are unrelated. This is because here one has $\xi_{y}^{2}=1$ in place of $\xi_{x}^{2}=1$. Therefore, the associated wave functions can be expressed just as in Eqs. (101) and (104) except that all the superscripts are changed from $x$ to $y$ and $\tau$ now labels $S_{y}(\mathbf{q}, \tau)$. However, the transformation of the $y$ components rather than the $x$ components requires replacing $\xi_{x}$ by $\xi_{y}$ which induces sign changes, so that

$$
\begin{aligned}
& m_{x}\left[\begin{array}{l}
\mathbf{O}_{n}^{(y, 1)} \\
\mathbf{O}_{n}^{(y, 2)}
\end{array}\right]=\left[\begin{array}{c}
-\mathbf{O}_{n}^{(y, 1)} \\
\mathbf{O}_{n}^{(y, 2)}
\end{array}\right], \\
& m_{y}\left[\begin{array}{l}
\mathbf{O}_{n}^{(y, 1)} \\
\mathbf{O}_{n}^{(y, 2)}
\end{array}\right]=\left[\begin{array}{c}
-\mathbf{O}_{n}^{(y, 2)} \\
\mathbf{O}_{n}^{(y, 1)}
\end{array}\right] .
\end{aligned}
$$

We want to construct wave functions in this sector which transform just like the $x$ components, so that they can be appropriately combined with the wave functions for the $x$ components. In view of Eq. (106), we set

$$
O_{n, \tau}^{(y, 1)}=O_{n, \tau}^{(x, 2)}, \quad O_{n, \tau}^{(y, 2)}=O_{n, \tau}^{(x, 1)} .
$$

So, the coefficients for $\mathbf{O}_{n}^{(y, 1)}$ are given by Eq. (104) and those for $\mathbf{O}_{n}^{(y, 2)}$ by Eq. (101). These wave functions are constructed to transform exactly as those for the $x$ components.

\section{3. $z$ components}

Similarly, we consider the effect of the transformations of the $z$ components. In this case, we take account of the factor $\xi_{z}$ to get

$$
\begin{gathered}
m_{x}\left[\begin{array}{l}
\mathbf{O}_{n}^{(z, 1)} \\
\mathbf{O}_{n}^{(z, 2)}
\end{array}\right]=\left[\begin{array}{c}
-\mathbf{O}_{n}^{(z, 1)} \\
\mathbf{O}_{n}^{(z, 2)}
\end{array}\right], \\
m_{y}\left[\begin{array}{l}
\mathbf{O}_{n}^{(z, 1)} \\
\mathbf{O}_{n}^{(z, 2)}
\end{array}\right]=\left[\begin{array}{c}
\mathbf{O}_{n}^{(z, 2)} \\
-\mathbf{O}_{n}^{(z, 1)}
\end{array}\right] .
\end{gathered}
$$

We now construct wave functions in this sector which transform just like the $x$ components. In view of Eq. (106), we set

$$
O_{n, \tau}^{(z, 1)}=O_{n, \tau}^{(x, 2)}, \quad O_{n, \tau}^{(z, 2)}=-O_{n, \tau}^{(x, 1)},
$$

So, the coefficients for $\mathbf{O}_{n}^{(z, 1)}$ are given by Eq. (104) and those for $\mathbf{O}_{n}^{(z, 2)}$ are the negatives of those of Eq. (101). These wave functions are constructed to transform exactly as those for the $x$ components.

\section{Total wave function and order parameters}

Now, we analyze the form of $\mathbf{M}^{(a b)}$ of Eq. (94) for $a \neq b$ using inversion symmetry. To do this, it is convenient to invoke invariance under the symmetry operation $m_{x} m_{y} \mathcal{I}$ whose effect is given in Table XV. We write

$$
m_{x} m_{y} \mathcal{I} S_{a}(\mathbf{q}, \tau)=\xi_{a}\left(m_{x}\right) \xi_{a}\left(m_{y}\right) S_{a}(\mathbf{q}, \mathcal{R} \tau)^{*},
$$

where $\mathcal{R} \tau=\tau$ for $\tau \neq 5,6,7,8$, otherwise $\mathcal{R} \tau=\tau \pm 2$ within the remaining sector of $\tau$ 's and $a$ (and later $b$ ) denotes one of $x$, $y$, and $z$. Thus, 


$$
\begin{aligned}
T \equiv & S_{a}(\mathbf{q}, \tau)^{*} M_{\tau \tau^{\prime}}^{(a b)} S_{b}\left(\mathbf{q}, \tau^{\prime}\right)=\left[m_{x} m_{y} \mathcal{I} S_{a}(\mathbf{q}, \tau)\right]^{*} M_{\tau \tau^{\prime}}^{(a b)} \\
& \times\left[m_{x} m_{y} \mathcal{I} S_{b}\left(\mathbf{q}, \tau^{\prime}\right)\right]=C_{a b} S_{a}(\mathbf{q}, \mathcal{R} \tau) M_{\tau \tau^{\prime}}^{(a b)} S_{b}\left(\mathbf{q}, \mathcal{R} \tau^{\prime}\right)^{*}
\end{aligned}
$$

where

$$
C_{a b}=\xi_{a}\left(m_{x}\right) \xi_{a}\left(m_{y}\right) \xi_{b}\left(m_{x}\right) \xi_{b}\left(m_{y}\right) .
$$

From the last line of Eq. (112), we deduce that

$$
M_{\mathcal{R} \tau^{\prime}, \mathcal{R} \tau}^{(b a)}=C_{a b} M_{\tau \tau^{\prime}}^{(a b)},
$$

or, since $\mathbf{M}$ is Hermitian that

$$
M_{\tau \tau^{\prime}}^{(a b)}=C_{a b}\left[M_{\mathcal{R}^{-1} \tau, \mathcal{R}^{-1} \tau^{\prime}}^{(a b)}\right]^{*} .
$$

Now, we consider the matrices $\mathbf{M}^{(a b)}$ in the symmetry adapted representation where

$$
\begin{aligned}
M_{n, m}^{(a b)} & =\sum_{\tau \tau^{\prime}}\left[O_{n \tau}^{a p}\right]^{*} M_{\tau \tau^{\prime}}^{(a b)} O_{m \tau^{\prime}}^{b p} \\
& =\sum_{\tau \tau^{\prime}} C_{a b}\left[O_{n \tau}^{a p}\right]^{*}\left[M_{\mathcal{R}^{-1} \tau, \mathcal{R}^{-1} \tau^{\prime}}^{(a b)}\right]^{*} O_{m \tau^{\prime}}^{b p} \\
& =C_{a b} \sum_{\tau \tau^{\prime}}\left[O_{n \mathcal{R} \tau}^{a p}\right]^{*}\left[M_{\tau, \tau^{\prime}}^{(a b)}\right]^{*} O_{m \mathcal{R} \tau^{\prime}}^{b p} .
\end{aligned}
$$

There are no matrix elements connecting $p$ and $p^{\prime} \neq p$ and the result is independent of $p$. One can verify from Eqs. (101) and (104) that

$$
O_{n, \mathcal{R} \tau}^{a p}=\left[O_{n, \tau}^{a p}\right]^{*},
$$

so that

$$
M_{n, m}^{(a b)}=C_{a b}\left(\left[O_{n \tau}^{a p}\right]^{*} M_{\tau, \tau^{\prime}}^{(a b)} O_{m \tau^{\prime}}^{b p}\right)^{*}=C_{a b}\left[M_{n m}^{a b}\right]^{*} .
$$

We have that $C_{x y}=-C_{x z}=-C_{y z}=1$, so that all the elements of $\mathbf{M}^{(x y)}$ are real and all the elements of $\mathbf{M}^{(x z)}$ and $\mathbf{M}^{(y z)}$ are imaginary. Thus, apart from an overall phase for the eigenfunction of each column, the phases of all the Fourier coefficients are fixed. What this means is that the critical eigenvector can be written as

$$
\psi=\sum_{p=1}^{2} \boldsymbol{\sigma}_{p} \sum_{n=1}^{6}\left(r_{n x} \mathbf{O}_{n}^{(x, p)}+r_{n y} \mathbf{O}_{n}^{(y, p)}+i r_{n z} \mathbf{O}_{n}^{(z, p)}\right),
$$

where the $r$ 's are all real valued and are normalized by

$$
\sum_{n=1}^{6} \sum_{\alpha}\left[r_{n \alpha}\right]^{2}=1
$$

and $\boldsymbol{\sigma}_{p}$ are arbitrary complex numbers. Thus, we have the result of Table XVI.

The order parameters are

$$
\boldsymbol{\sigma}_{1} \equiv \sigma_{1} e^{-i \phi_{1}}, \quad \boldsymbol{\sigma}_{2} \equiv \sigma_{2} e^{-i \phi_{2}}
$$

\begin{tabular}{|c|c|c|}
\hline Spin & $\boldsymbol{\sigma}_{1}$ & $\boldsymbol{\sigma}_{2}$ \\
\hline \multirow[t]{3}{*}{$\mathbf{S}(\mathbf{q}, 1)$} & $r_{1 x}$ & $r_{2 x}$ \\
\hline & $r_{1 y}$ & $r_{2 y}$ \\
\hline & $i r_{1 z}$ & $i r_{2 z}$ \\
\hline \multirow[t]{3}{*}{$\mathbf{S}(\mathbf{q}, 2)$} & $r_{2 x}$ & $r_{1 x}$ \\
\hline & $r_{2 y}$ & $r_{1 y}$ \\
\hline & $-i r_{2 z}$ & $-i r_{1 z}$ \\
\hline \multirow[t]{3}{*}{$\mathbf{S}(\mathbf{q}, 3)$} & $r_{1 x}$ & $-r_{2 x}$ \\
\hline & $-r_{1 y}$ & $r_{2 y}$ \\
\hline & $-i r_{1 z}$ & $i r_{2 z}$ \\
\hline \multirow[t]{3}{*}{$\mathbf{S}(\mathbf{q}, 4)$} & $r_{2 x}$ & $-r_{1 x}$ \\
\hline & $-r_{2 y}$ & $r_{1 y}$ \\
\hline & $i r_{2 z}$ & $-i r_{1 z}$ \\
\hline \multirow[t]{3}{*}{$\mathbf{S}(\mathbf{q}, 5)$} & $z_{x}$ & $-z_{x}$ \\
\hline & $-z_{y}$ & $z_{y}$ \\
\hline & $i z_{z}$ & $i z_{z}$ \\
\hline \multirow[t]{3}{*}{$\mathbf{S}(\mathbf{q}, 6)$} & $z_{x}$ & $z_{x}$ \\
\hline & $z_{y}$ & $z_{y}$ \\
\hline & $-i z_{z}$ & $i z_{z}$ \\
\hline \multirow[t]{2}{*}{$\mathbf{S}(\mathbf{q}, 7)$} & $z_{x_{*}}^{*}$ & $-z_{x}^{*}$ \\
\hline & $\begin{array}{l}-z_{y} \\
i z_{z}^{*}\end{array}$ & $\begin{array}{c}z_{y_{*}} \\
i z_{z}\end{array}$ \\
\hline \multirow[t]{2}{*}{$\mathbf{S}(\mathbf{q}, 8)$} & $z_{x}^{*}$ & $z_{x}^{*}$ \\
\hline & $\begin{array}{c}z_{y} \\
-i z^{*}\end{array}$ & $\begin{array}{l}z_{y} \\
i z^{*}\end{array}$ \\
\hline \multirow[t]{3}{*}{$\mathbf{S}(\mathbf{q}, 9)$} & $r_{5 x}$ & $r_{6 x}$ \\
\hline & $r_{5 y}$ & $r_{6 y}$ \\
\hline & $i r_{5 z}$ & $i r_{6 z}$ \\
\hline \multirow[t]{3}{*}{$\mathbf{S}(\mathbf{q}, 10)$} & $r_{6 x}$ & $r_{5 x}$ \\
\hline & $r_{6 y}$ & $r_{5 y}$ \\
\hline & $-i r_{6 z}$ & $-i r_{5 z}$ \\
\hline \multirow[t]{3}{*}{$\mathbf{S}(\mathbf{q}, 11)$} & $r_{5 x}$ & $-r_{6 x}$ \\
\hline & $-r_{5 y}$ & $r_{6 y}$ \\
\hline & $-i r_{5 z}$ & $i r_{6 z}$ \\
\hline \multirow[t]{3}{*}{$\mathbf{S}(\mathbf{q}, 12)$} & $r_{6 x}$ & $-r_{5 x}$ \\
\hline & $-r_{6 y}$ & $r_{5 y}$ \\
\hline & $i r_{6 z}$ & $-i r_{5 z}$ \\
\hline
\end{tabular}

Neither the relative magnitudes of $\boldsymbol{\sigma}_{1}$ and $\boldsymbol{\sigma}_{2}$ nor their phases are fixed by the quadratic terms within the Landau expansion. Note that the structure parameters of Table XVI are determined by the microscopic interactions which determine the matrix elements in the quadratic free energy. (Since
TABLE XVI. Normalized spin functions (i.e., Fourier coefficients) within the unit cell of $\mathrm{TbMn}_{2} \mathrm{O}_{5}$ for wave vector $\left(\frac{1}{2}, 0, q\right)$. Here, $z_{\alpha}=\left(r_{3 \alpha}+i r_{4 \alpha}\right) / \sqrt{2}$. All the $r$ 's are real variables. The wave function listed under $\sigma_{1}\left(\sigma_{2}\right)$ transforms according to the first (second) column of the irrep. The actual spin structure is a linear combination of the two columns with arbitrary complex coefficients.

these are usually not well known, one has recourse to a symmetry analysis.) The direction in $\boldsymbol{\sigma}_{1}-\boldsymbol{\sigma}_{2}$ space which the system assumes is determined by fourth- or higher-order terms in the Landau expansion. Since not much is known about these terms, this direction is reasonably treated as a 
parameter to be extracted from the experimental data. We use Table XVI to write the most general spin functions consistent with crystal symmetry. For instance, we write

$$
\begin{aligned}
\mathbf{S}(\mathbf{R}, 1)= & \frac{1}{2} \boldsymbol{\sigma}_{1}\left[r_{1 x} \hat{i}+r_{1 y} \hat{j}+i r_{1 z} \hat{k}\right] e^{-i \mathbf{q} \cdot \mathbf{R}}+\text { c.c. }+\frac{1}{2} \boldsymbol{\sigma}_{2}\left[r_{2 x} \hat{i}\right. \\
& \left.+r_{2 y} \hat{j}+i r_{2 z} \hat{k}\right] e^{-i \mathbf{q} \cdot \mathbf{R}}+\text { c.c. }
\end{aligned}
$$

Using this and similar equations for the other sublattices, we find that

$$
\begin{aligned}
\mathbf{S}(\mathbf{R}, 1)= & \sigma_{1}\left[\left(r_{1 x} \hat{i}+r_{1 y} \hat{j}\right) \cos \left(\mathbf{q} \cdot \mathbf{R}+\phi_{1}\right)\right. \\
& \left.+r_{1 z} \hat{k} \sin \left(\mathbf{q} \cdot \mathbf{R}+\phi_{1}\right)\right]+\sigma_{2}\left[\left(r_{2 x} \hat{i}+r_{2 y} \hat{j}\right)\right. \\
& \left.\times \cos \left(\mathbf{q} \cdot \mathbf{R}+\phi_{2}\right)+r_{2 z} \hat{k} \sin \left(\mathbf{q} \cdot \mathbf{R}+\phi_{2}\right)\right], \\
\mathbf{S}(\mathbf{R}, 2)= & \sigma_{1}\left[\left(r_{2 x} \hat{i}+r_{2 y} \hat{j}\right) \cos \left(\mathbf{q} \cdot \mathbf{R}+\phi_{1}\right)\right. \\
& \left.-r_{2 z} \hat{k} \sin \left(\mathbf{q} \cdot \mathbf{R}+\phi_{1}\right)\right]+\sigma_{2}\left[\left(r_{1 x} \hat{i}+r_{1 y} \hat{j}\right)\right. \\
& \left.\times \cos \left(\mathbf{q} \cdot \mathbf{R}+\phi_{2}\right)-r_{1 z} \hat{k} \sin \left(\mathbf{q} \cdot \mathbf{R}+\phi_{2}\right)\right],
\end{aligned}
$$

$\mathbf{S}(\mathbf{R}, 3)=\sigma_{1}\left[\left(r_{1 x} \hat{i}-r_{1 y} \hat{j}\right) \cos \left(\mathbf{q} \cdot \mathbf{R}+\phi_{1}\right)\right.$

$$
\begin{aligned}
& \left.-r_{1 z} \hat{k} \sin \left(\mathbf{q} \cdot \mathbf{R}+\phi_{1}\right)\right]+\sigma_{2}\left[\left(-r_{2 x} \hat{i}+r_{2 y} \hat{j}\right)\right. \\
& \left.\times \cos \left(\mathbf{q} \cdot \mathbf{R}+\phi_{2}\right)+r_{2 z} \hat{k} \sin \left(\mathbf{q} \cdot \mathbf{R}+\phi_{2}\right)\right],
\end{aligned}
$$

$$
\begin{aligned}
\mathbf{S}(\mathbf{R}, 4)= & \sigma_{1}\left[\left(r_{2 x} \hat{i}-r_{2 y} \hat{j}\right) \cos \left(\mathbf{q} \cdot \mathbf{R}+\phi_{1}\right)\right. \\
& \left.+r_{2 z} \hat{k} \sin \left(\mathbf{q} \cdot \mathbf{R}+\phi_{1}\right)\right]+\sigma_{2}\left[\left(-r_{1 x} \hat{i}+r_{1 y} \hat{j}\right)\right. \\
& \left.\times \cos \left(\mathbf{q} \cdot \mathbf{R}+\phi_{2}\right)-r_{1 z} \hat{k} \sin \left(\mathbf{q} \cdot \mathbf{R}+\phi_{2}\right)\right],
\end{aligned}
$$

$$
\begin{aligned}
\mathbf{S}(\mathbf{R}, 5)= & \sigma_{1}\left[\left(z_{x}^{\prime} \hat{i}-z_{y}^{\prime} \hat{j}-z_{z}^{\prime \prime} \hat{k}\right) \cos \left(\mathbf{q} \cdot \mathbf{R}+\phi_{1}\right)\right. \\
& \left.+\left(z_{x}^{\prime \prime} \hat{i}-z_{y}^{\prime \prime} \hat{j}+z_{z}^{\prime} \hat{k}\right) \sin \left(\mathbf{q} \cdot \mathbf{R}+\phi_{1}\right)\right] \\
& +\sigma_{2}\left[\left(-z_{x}^{\prime} \hat{i}+z_{y}^{\prime} \hat{j}-z_{z}^{\prime \prime} \hat{k}\right) \cos \left(\mathbf{q} \cdot \mathbf{R}+\phi_{2}\right)\right. \\
& \left.+\left(-z_{x}^{\prime \prime} \hat{i}+z_{y}^{\prime \prime} \hat{j}+z_{z}^{\prime} \hat{k}\right) \sin \left(\mathbf{q} \cdot \mathbf{R}+\phi_{2}\right)\right]
\end{aligned}
$$

$$
\begin{aligned}
\mathbf{S}\left(\mathbf{R}_{6}\right)= & \sigma_{1}\left[\left(z_{x}^{\prime} \hat{i}+z_{y}^{\prime} \hat{j}+z_{z}^{\prime \prime} \hat{k}\right) \cos \left(\mathbf{q} \cdot \mathbf{R}+\phi_{1}\right)\right. \\
& \left.+\left(z_{x}^{\prime \prime} \hat{i}+z_{y}^{\prime \prime} \hat{j} i-z_{z}^{\prime} \hat{k}\right) \sin \left(\mathbf{q} \cdot \mathbf{R}+\phi_{1}\right)\right] \\
& +\sigma_{2}\left[\left(z_{x}^{\prime} \hat{i}+z_{y}^{\prime} \hat{j}-z_{z}^{\prime \prime} \hat{k}\right) \cos \left(\mathbf{q} \cdot \mathbf{R}+\phi_{2}\right)\right. \\
& \left.+\left(z_{x}^{\prime \prime} \hat{i}+z_{y}^{\prime \prime} \hat{j}+z_{z}^{\prime} \hat{k}\right) \sin \left(\mathbf{q} \cdot \mathbf{R}+\phi_{2}\right)\right]
\end{aligned}
$$

$$
\begin{aligned}
\mathbf{S}(\mathbf{R}, 7)= & \sigma_{1}\left[\left(z_{x}^{\prime} \hat{i}-z_{y}^{\prime} \hat{j}+z_{z}^{\prime \prime} \hat{k}\right) \cos \left(\mathbf{q} \cdot \mathbf{R}+\phi_{1}\right)\right. \\
& \left.+\left(-z_{x}^{\prime \prime} \hat{i}+z_{y}^{\prime \prime} \hat{j}+z_{z}^{\prime} \hat{k}\right) \sin \left(\mathbf{q} \cdot \mathbf{R}+\phi_{1}\right)\right] \\
& +\sigma_{2}\left[\left(-z_{x}^{\prime} \hat{i}+z_{y}^{\prime} \hat{j}+z_{z}^{\prime \prime} \hat{k}\right) \cos \left(\mathbf{q} \cdot \mathbf{R}+\phi_{2}\right)\right. \\
& \left.+\left(z_{x}^{\prime \prime} \hat{i}-z_{y}^{\prime \prime} \hat{j}+z_{z}^{\prime} \hat{k}\right) \sin \left(\mathbf{q} \cdot \mathbf{R}+\phi_{2}\right)\right],
\end{aligned}
$$

$$
\begin{aligned}
\mathbf{S}\left(\mathbf{R}_{8}\right)= & \sigma_{1}\left[\left(z_{x}^{\prime} \hat{i}+z_{y}^{\prime} \hat{j}-z_{z}^{\prime \prime} \hat{k}\right) \cos \left(\mathbf{q} \cdot \mathbf{R}+\phi_{1}\right)\right. \\
& \left.+\left(-z_{x}^{\prime \prime} \hat{i}-z_{y}^{\prime \prime} \hat{j}-z_{z}^{\prime} \hat{k}\right) \sin \left(\mathbf{q} \cdot \mathbf{R}+\phi_{1}\right)\right] \\
& +\sigma_{2}\left[\left(z_{x}^{\prime} \hat{i}+z_{y}^{\prime} \hat{j}+z_{z}^{\prime \prime} \hat{k}\right) \cos \left(\mathbf{q} \cdot \mathbf{R}+\phi_{2}\right)\right. \\
& \left.+\left(-z_{x}^{\prime \prime} \hat{i}-z_{y}^{\prime \prime} \hat{j}+z_{z}^{\prime} \hat{k}\right) \sin \left(\mathbf{q} \cdot \mathbf{R}+\phi_{2}\right)\right],
\end{aligned}
$$$$
\mathbf{S}(\mathbf{R}, 9)=\sigma_{1}\left[\left(r_{5 x} \hat{i}+r_{5 y} \hat{j}\right) \cos \left(\mathbf{q} \cdot \mathbf{R}+\phi_{1}\right)\right.
$$$$
\left.+r_{5 z} \hat{k} \sin \left(\mathbf{q} \cdot \mathbf{R}+\phi_{1}\right)\right]
$$$$
+\sigma_{2}\left[\left(r_{6 x} \hat{i}+r_{6 y} \hat{j}\right) \cos \left(\mathbf{q} \cdot \mathbf{R}+\phi_{2}\right)\right.
$$$$
\left.+r_{6 z} \hat{k} \sin \left(\mathbf{q} \cdot \mathbf{R}+\phi_{2}\right)\right] \text {, }
$$$$
\mathbf{S}(\mathbf{R}, 10)=\sigma_{1}\left[\left(r_{6 x} \hat{i}+r_{6 y} \hat{j}\right) \cos \left(\mathbf{q} \cdot \mathbf{R}+\phi_{1}\right)\right.
$$$$
\left.-r_{6 z} \hat{k} \sin \left(\mathbf{q} \cdot \mathbf{R}+\phi_{1}\right)\right]
$$$$
+\sigma_{2}\left[\left(r_{5 x} \hat{i}+r_{5 y} \hat{j}\right) \cos \left(\mathbf{q} \cdot \mathbf{R}+\phi_{2}\right)\right.
$$$$
\left.-r_{5 z} \hat{k} \sin \left(\mathbf{q} \cdot \mathbf{R}+\phi_{2}\right)\right] \text {, }
$$

$$
\begin{aligned}
\mathbf{S}(\mathbf{R}, 11)= & \sigma_{1}\left[\left(r_{5 x} \hat{i}-r_{5 y} \hat{j}\right) \cos \left(\mathbf{q} \cdot \mathbf{R}+\phi_{1}\right)\right. \\
& \left.-r_{5 z} \hat{k} \sin \left(\mathbf{q} \cdot \mathbf{R}+\phi_{1}\right)\right] \\
& +\sigma_{2}\left[\left(-r_{6 x} \hat{i}+r_{6 y} \hat{j}\right) \cos \left(\mathbf{q} \cdot \mathbf{R}+\phi_{2}\right)\right. \\
& \left.+r_{6 z} \hat{k} \sin \left(\mathbf{q} \cdot \mathbf{R}+\phi_{2}\right)\right], \\
\mathbf{S}(\mathbf{R}, 12)= & \sigma_{1}\left[\left(r_{6 x} \hat{i}-r_{6 y} \hat{j}\right) \cos \left(\mathbf{q} \cdot \mathbf{R}+\phi_{1}\right)\right. \\
& \left.+r_{6 z} \hat{k} \sin \left(\mathbf{q} \cdot \mathbf{R}+\phi_{1}\right)\right] \\
& +\sigma_{2}\left[\left(-r_{5 x} \hat{i}+r_{5 y} \hat{j}\right) \cos \left(\mathbf{q} \cdot \mathbf{R}+\phi_{2}\right)\right. \\
& \left.-r_{5 z} \hat{k} \sin \left(\mathbf{q} \cdot \mathbf{R}+\phi_{2}\right)\right] .
\end{aligned}
$$

In Table XVI, the position of each spin is $\mathbf{R}+\boldsymbol{\tau}_{n}$, where the $\boldsymbol{\tau}$ are listed in Table XIV and $\mathbf{R}$ is a Bravais lattice vector. The symmetry properties of the order parameters are

$$
\begin{gathered}
m_{x}\left[\begin{array}{l}
\boldsymbol{\sigma}_{1} \\
\boldsymbol{\sigma}_{2}
\end{array}\right]=\left[\begin{array}{c}
\boldsymbol{\sigma}_{1} \\
-\boldsymbol{\sigma}_{2}
\end{array}\right], \\
m_{y}\left[\begin{array}{l}
\boldsymbol{\sigma}_{1} \\
\boldsymbol{\sigma}_{2}
\end{array}\right]=\left[\begin{array}{c}
\boldsymbol{\sigma}_{2} \\
-\boldsymbol{\sigma}_{1}
\end{array}\right], \\
\mathcal{I}\left[\begin{array}{l}
\boldsymbol{\sigma}_{1} \\
\boldsymbol{\sigma}_{2}
\end{array}\right]=\left[\begin{array}{c}
\boldsymbol{\sigma}_{2}^{*} \\
\boldsymbol{\sigma}_{1}^{*}
\end{array}\right] .
\end{gathered}
$$

We now check a few representative cases of the above transformation. If we apply $m_{x}$ to $S(\mathbf{q}, 1)$, we do not change the signs of the $x$ component but do change the signs of the $y$ and $z$ components. As a result, we get $S(\mathbf{q}, 3)$ except that $\sigma_{y}$ has changed sign, in agreement with the first line of Eq. (124). If we apply $m_{y}$ to $S(\mathbf{q}, 1)$, we do not change the sign of the $y$ component but do change the signs of the $x$ and $z$ components. As a result, we get $S(\mathbf{q}, 4)$ except that now $\boldsymbol{\sigma}_{1}$ is 
replaced by $\boldsymbol{\sigma}_{2}$ and $\boldsymbol{\sigma}_{2}$ is replaced by $-\boldsymbol{\sigma}_{1}$, in agreement with the second line of Eq. (124). When inversion is applied to $S(\mathbf{q}, 1)$, we change the sign of $\mathbf{R}$ but not the orientation of the spins which are pseudovectors. We then obtain $S(\mathbf{q}, 2)$ provided we replace $\boldsymbol{\sigma}_{1}$ by $\boldsymbol{\sigma}_{2}^{*}$ and $\boldsymbol{\sigma}_{2}$ by $\boldsymbol{\sigma}_{1}^{*}$, in agreement with the last line of Eq. (124).

\section{Comparison to group theory}

Here, I briefly compare the above calculation to the one using the standard formulation of representation theory. The first step in the standard formulation is to find the irreps of the group of the wave vector. The easiest way to do this is to introduce a double group having eight elements (see Appendix B) since we need to take account of the operator $m_{y}^{2} \equiv$ $-E$. (This is done in Appendix B.) From this, one finds that each Wyckoff orbit and each spin component can be considered separately (since they do not transform into one another under the operations we consider). Then, in every case the only irrep that appears is the two-dimensional one for which we set

$$
m_{x}=\left[\begin{array}{cc}
1 & 0 \\
0 & -1
\end{array}\right], \quad m_{y}=\left[\begin{array}{cc}
0 & 1 \\
-1 & 0
\end{array}\right], \quad m_{x} m_{y}=\left[\begin{array}{ll}
0 & 1 \\
1 & 0
\end{array}\right] .
$$

Indeed, one can verify that the functions in the second (third) column of Table XVI comprise a basis vector for column one (two) of this two-dimensional irrep. One might ask: "Why have we undertaken the ugly detailed consideration of the matrix for $F_{2}$ ?" The point is that within standard representation theory, all the variables in Table XVI would be independently assigned arbitrary phases. In addition, the amplitudes for the Tb orbits (sublattices 5 and 6 and sublattices 7 and 8) would have independent amplitudes. To get the results actually shown in Table XVI, one would have to do the equivalent of analyzing the effect of inversion invariance of the free energy. This task would be a very technical exercise in the arcane aspects of group theory which here we avoid by an exercise in algebra, which, though messy, is basically high school math. I also warn the reader that canned programs to perform the standard representation analysis cannot always be relied upon to be correct. It is worth noting that published papers dealing with TMO25 have not invoked inversion symmetry. For instance, in Ref. 55 one sees the statement "As in the incommensurate case, ${ }^{3}$ each of the magnetic atoms in the unit cell is allowed to have an independent SDW, i.e., its own amplitude and phase," and later on in Ref. 56, "all phases were subsequently fixed... to be rational fractions of $\pi$." Use of the present theory would eliminate most of the phases and would relate the two distinct $\mathrm{Mn}^{4+}$ Wyckoff orbits (just as happened for TMO).

Finally, to see the effect of inversion on a concrete level, I consider the upper right and lower left $4 \times 4$ submatrices of $\mathbf{M}^{(x x)}$, which are denoted $\mathbf{M}_{\mathrm{ur}}$ and $\mathbf{M}_{\mathrm{ll}}$, respectively. If we do not use inversion symmetry (this amounts to following the usual group theoretical formulation), these matrices assume the form

$$
\begin{aligned}
\mathbf{M}_{\mathrm{ur}} & =\left[\begin{array}{cccc}
a & b & c & d \\
b & a & -d & -c \\
c & d & a & b \\
-d & -c & b & a
\end{array}\right], \\
\mathbf{M}_{11} & =\left[\begin{array}{cccc}
a^{*} & b^{*} & c^{*} & -d^{*} \\
b^{*} & a^{*} & d^{*} & -c^{*} \\
c^{*} & -d^{*} & a^{*} & b^{*} \\
d^{*} & -c^{*} & b^{*} & a^{*}
\end{array}\right],
\end{aligned}
$$

where now all these parameters are complex valued. (Previously, in Eq. (95) all these parameters were real valued.) From these results, one could again introduce the wave functions of Eq. (101). However, in this case, the matrix elements appearing in the analog of Eq. (102) would not be real. In fact, Eq. (126) indicates that in Eq. (102) the quantities $a, b$, $c$, and $d$ in the upper right sector of the matrix would be complex and those in the lower left sector would be replaced by their complex conjugates (to ensure Hermiticity). Thus, invoking inversion symmetry does not change the symmetry adapted coordinates of Eq. (101). Rather, it fixes the phases so that the result can be expressed in terms of real-valued parameters, as we have done in Table XVI.

\section{Comparison to $\mathrm{YMn}_{2} \mathrm{O}_{5}$}

$\mathrm{YMn}_{2} \mathrm{O}_{5}(\mathrm{YMO} 25)$ is isostructural to TMO25, so its magnetic structure is relevant to the present discussion. I will consider the highest-temperature magnetically ordered phase, which appears between about 20 and $45 \mathrm{~K}$. In this compound, $\mathrm{Y}$ is nonmagnetic and in the higher-temperature ordered phase $q_{z}=1 / 4$, so the system is commensurate. However, since the value of $q_{z}$ is not special, the symmetry of this state is essentially the same as that of TMO25. Throughout this section, the structural information is taken from Fig. 2 of Ref. 58. (The uppermost panel is mislabeled and is obviously the one we want for the highest-temperature ordered phase.)

In Fig. 7, we see that the spin wave function is an eigenvector of $m_{x}$ with eigenvalue -1 . So, this structure must be that of the second column of the irrep. In accordance with this identification, one sees that the initial wave function is orthogonal to the wave function transformed by $m_{y}$ (since this transformation will produce a wave function associated with the first column). Referring to Eq. (123), one sees that to describe the pattern of $\mathrm{Mn}^{3+}$ spins, one chooses

$$
\begin{gathered}
\boldsymbol{\sigma}_{1}=0, \quad r_{2 x}=-r_{1 x} \approx 0.95, \\
r_{1 y}=-r_{2 y} \approx 0.3 .
\end{gathered}
$$

The point we make here is that $\boldsymbol{\sigma}_{1}=0$. Although the values of these order parameters were not given in Ref. 58, it seems clear that in the lower-temperature phase the order parameters are comparable in magnitude. ${ }^{59}$

\section{D. $\mathrm{CuFeO}_{2}$}

The magnetic phase diagram of $\mathrm{CuFeO}_{2}$ has been investigated continually over the last decade or so. Early 

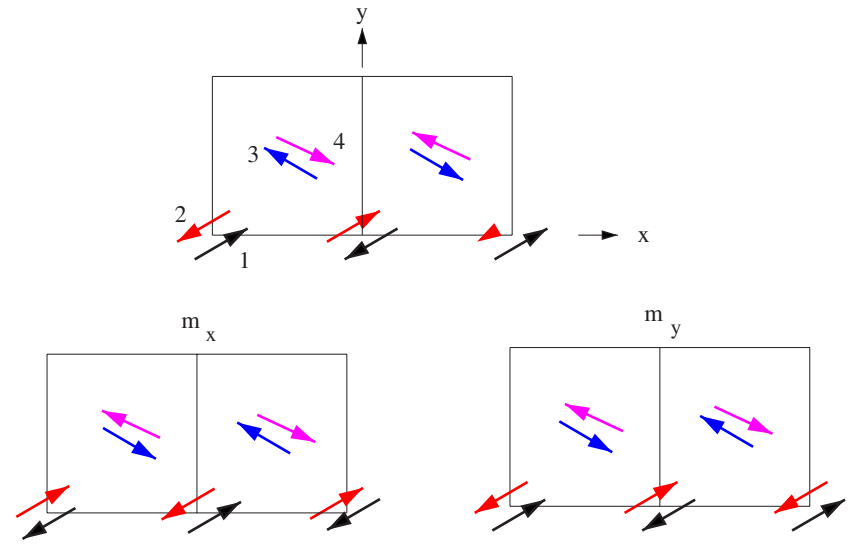

FIG. 7. (Color online) Top: The spin structure of the $\mathrm{Mn}^{3+}$ ions in $\mathrm{YMn}_{2} \mathrm{O}_{5}$ (limited to one a-b plane), taken from Fig. 2 of Ref. 58. The sublattices are labeled in our convention. Bottom left: The spin structure after transformation by $m_{x}$. Bottom right: The spin structure after transformation by $m_{y}$.

studies ${ }^{60,61}$ showed a rich phase diagram and these combined with magnetoelectric data ${ }^{10}$ led to the phase diagram for magnetic fields up to about $15 \mathrm{~T}$ given in Ref. 10 which is reproduced in Fig. 8.

Above $T_{N 2} \approx 10 \mathrm{~K}$, the crystal structure is that of space group of $R \overline{3} m$ (Ref. 62) (No. 166 in Ref. 33). Below that temperature, there is apparently a very small lattice distortion which gives rise to a lower symmetry crystal structure. ${ }^{63,64}$

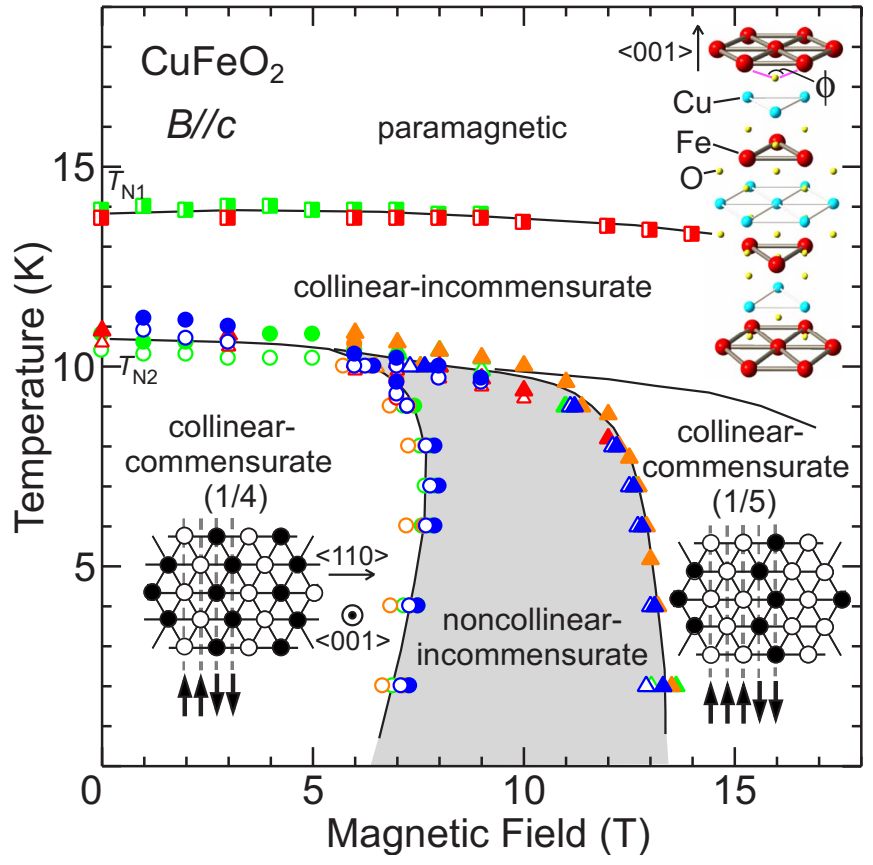

FIG. 8. (Color online) Temperature $(T)$ versus magnetic field (B) phase diagram of $\mathrm{CuFeO}_{2}$ with $B$ applied along the $\mathbf{c}$ axis from Kimura et al. (Ref. 10). The upper inset shows the crystal structure of $\mathrm{CuFeO}_{2}$ and the lower insets show the magnetic structure of the commensurate states, where white and black circles correspond to the positive and negative $c$ directions. Note in the lower left inset that the hexagonal $\langle 110\rangle$ direction (along which $q$ is oriented) is a nearest neighbor direction.
TABLE XVII. General positions for $R \overline{3} m$, with respect to rhombohedral axes $\mathbf{a}_{n}$, where $\mathbf{a}_{1}=-(a / 2) \hat{i}-(a \sqrt{3} / 6) \hat{j}+c \hat{k}, \mathbf{a}_{2}=(a / 2) \hat{i}$ $-(a \sqrt{3} / 6) \hat{j}+c \hat{k}$, and $\mathbf{a}_{3}=(a \sqrt{3} / 3) \hat{j}+c \hat{k}$, where $c$ is the distance between neighbor planes of $\mathrm{Fe}$ ions and $a$ is the separation between nearest neighbors in the plane. Here, " 3 " denotes a threefold rotation and $m_{n}$ labels the three mirror planes which contain the threefold axis and $\mathbf{a}_{n}$.

\begin{tabular}{lcc}
\hline \hline$E \mathbf{r}=(x, y, z)$ & $3 \mathbf{r}=(z, x, y)$ & $3^{2} \mathbf{r}=(y, z, x)$ \\
$m_{3} \mathbf{r}=(y, x, z)$ & $m_{2} \mathbf{r}=(z, y, x)$ & $m_{1} \mathbf{r}=(x, z, y)$ \\
$\mathcal{I} \mathbf{r}=(\bar{x}, \bar{y}, \bar{z})$ & $\mathcal{I} 3 \mathbf{r}=(\bar{z}, \bar{x}, \bar{y})$ & $\mathcal{I} 3^{2} \mathbf{r}=(\bar{y}, \bar{z}, \bar{x})$ \\
$\mathcal{I} m_{3} \mathbf{r}=(\bar{y}, \bar{x}, \bar{z})$ & $\mathcal{I} m_{2} \mathbf{r}=(\bar{z}, \bar{y}, \bar{x})$ & $\mathcal{I} m_{1} \mathbf{r}=(\bar{x}, \bar{z}, \bar{y})$ \\
\hline \hline
\end{tabular}

However, since this distortion may not be essential to explaining the appearance of ferroelectricity, ${ }^{65}$ we will ignore the presence of this lattice distortion. The general positions of ions within space group $R \overline{3} m$ are given in Table XVII.

Our analysis is based on the following logic (refer to the phase diagram of Fig. 8). We assume that as the temperature is lowered in a magnetic field of about $10 \mathrm{~T}$, the continuous transition from the paramagnetic phase to the collinear incommensurate (CIC) phase introduces a single irrep which we will identify by our simple method. Then, further lowering of the temperature will introduce a second irrep, taking us into the noncollinear incommensurate (NIC) phase whose symmetry and ferroelectricity we wish to discuss. Both these phases are characterized by an incommensurate wave vector along a hexagonal $\langle 110\rangle$ direction, which is the direction to a nearest neighbor in the triangular lattice plane, as shown in Fig. 8. As mentioned, although, in principle, the lattice distortion does break the threefold symmetry, we will assume that the three states which are related by the threefold rotation have only slightly different energies in the distorted structure and our arguments have to be understood in that sense.

We assume the $R \overline{3} m$ space group and are interested in structures associated with a wave vector in the star of $\mathbf{q}_{1}$ $\equiv\langle q, q, 0\rangle$ (referred to hexagonal axes). These wave vectors are parallel to a nearest neighbor vectors of the triangular plane of Fe ions. Consider the wave vector $\mathbf{q}_{1} \equiv q \hat{i}$. The only operation (other than the identity) that conserves wave vector is $2_{x}$, a twofold rotation about the axis of the wave vector $\left(2_{x}=\mathcal{I} m_{3}\right)$. Clearly, the Fourier component $m_{x}(\mathbf{q})$ obeys

$$
2_{x} m_{x}\left(\mathbf{q}_{1}\right)=\lambda\left(2_{x}\right) m_{x}\left(\mathbf{q}_{1}\right),
$$

with $\lambda\left(2_{x}\right)=1$, and we call this irrep $\Gamma_{1}$. For irrep $\Gamma_{2}$, we have

$$
\begin{aligned}
& 2_{x} m_{y}\left(\mathbf{q}_{1}\right)=\lambda\left(2_{x}\right) m_{y}\left(\mathbf{q}_{1}\right), \\
& 2_{x} m_{z}\left(\mathbf{q}_{1}\right)=\lambda\left(2_{x}\right) m_{z}\left(\mathbf{q}_{1}\right),
\end{aligned}
$$

but with $\lambda\left(2_{x}\right)=-1$.

So far, the phases of the complex Fourier coefficients are not fixed. To do that, we consider the effect of inversion, which leads to 


$$
\operatorname{Im}_{\alpha}\left(\mathbf{q}_{1}\right)=m_{\alpha}\left(\mathbf{q}_{1}\right)^{*} .
$$

To fix the phases in irrep $\Gamma_{2}$, we note that its quadratic free energy can be expressed as

$$
\begin{aligned}
F_{2}= & A\left|m_{y}\left(\mathbf{q}_{1}\right)\right|^{2}+B\left|m_{z}\left(\mathbf{q}_{1}\right)\right|^{2}+C m_{y}\left(\mathbf{q}_{1}\right)^{*} m_{z}\left(\mathbf{q}_{1}\right) \\
& +C^{*} m_{z}\left(\mathbf{q}_{1}\right)^{*} m_{y}\left(\mathbf{q}_{1}\right),
\end{aligned}
$$

where $A$ and $B$ are real and $C$ is complex. Using the fact that $F_{2}$ must be invariant under $\mathcal{I}$, we write

$$
\begin{aligned}
F_{2}= & A\left|m_{y}\left(\mathbf{q}_{1}\right)\right|^{2}+B\left|m_{z}\left(\mathbf{q}_{1}\right)\right|^{2}+C m_{y}\left(\mathbf{q}_{1}\right) m_{z}\left(\mathbf{q}_{1}\right)^{*} \\
& +C^{*} m_{z}\left(\mathbf{q}_{1}\right) m_{y}\left(\mathbf{q}_{1}\right)^{*} .
\end{aligned}
$$

Comparing this with Eq. (131), we conclude that $C$ has to be real. Since the $m$ 's can be complex, this means that the two components of the eigenvector of the quadratic form [i.e., $m_{y}\left(\mathbf{q}_{1}\right)$ and $\left.m_{z}\left(\mathbf{q}_{1}\right)\right]$ have to have the same complex phase.

We now introduce order parameters which describe the magnitude and phase of these two symmetry labels (irreps) which make up the wave function. When both irreps are present, one has

$$
m_{x}\left(\mathbf{q}_{1}\right)=\boldsymbol{\sigma}_{1}\left(\mathbf{q}_{1}\right)
$$

and

$$
m_{y}\left(\mathbf{q}_{1}\right)=\boldsymbol{\sigma}_{2}\left(\mathbf{q}_{1}\right) r, \quad m_{z}\left(\mathbf{q}_{1}\right)=\boldsymbol{\sigma}_{2}\left(\mathbf{q}_{1}\right) s,
$$

where $r^{2}+s^{2}=1$ and $\boldsymbol{\sigma}_{n}\left( \pm\left|\mathbf{q}_{k}\right|\right)=\sigma_{n} e^{\mp i \phi_{n}}$. We have the transformation properties

$$
\begin{array}{cc}
2_{x} \boldsymbol{\sigma}_{1}\left(\mathbf{q}_{1}\right)=\boldsymbol{\sigma}_{1}\left(\mathbf{q}_{1}\right), & 2_{x} \boldsymbol{\sigma}_{2}\left(\mathbf{q}_{1}\right)=-\boldsymbol{\sigma}_{2}\left(\mathbf{q}_{1}\right), \\
\mathcal{I} \boldsymbol{\sigma}_{1}\left(\mathbf{q}_{1}\right)=\left[\boldsymbol{\sigma}_{1}\left(\mathbf{q}_{1}\right)\right]^{*}, & \mathcal{I} \boldsymbol{\sigma}_{2}\left(\mathbf{q}_{1}\right)=\left[\boldsymbol{\sigma}_{2}\left(\mathbf{q}_{1}\right)\right]^{*} .
\end{array}
$$

(Note that the phases $\phi_{n}$ are fixed by the fourth-order terms in the free energy to be the same for all members of the star of the wave vector.) Thus, when both irreps (of $\mathbf{q}_{1}$ ) are present, we have (redefining the order parameters to remove a factor of 2)

$$
\begin{gathered}
m_{x}(\mathbf{r})=\sigma_{1}\left(\mathbf{q}_{1}\right) \cos \left(q x+\phi_{1}\right), \\
m_{y}(\mathbf{r})=\sigma_{2}\left(\mathbf{q}_{1}\right) r \cos \left(q x+\phi_{2}\right), \\
m_{z}(\mathbf{r})=\sigma_{2}\left(\mathbf{q}_{1}\right) s \cos \left(q x+\phi_{2}\right),
\end{gathered}
$$

where $q=\left|\mathbf{q}_{1}\right|$.

We apply these results as follows. As one lowers the temperature from the paramagnetic phase, we assume that we first enter the CIC which has the spins predominantly along the $z$ axis. Therefore, in this phase we assume that only irrep $\Gamma_{2}$ is active. Notice that in this phase, the spins will not lie exactly along the $z$ axis. Indeed, recent work ${ }^{66}$ indicates that this phase is one in which the amplitudes are sinusoidally modulated and the spins are oriented in the $y-z$ plane (as described by irrep $\Gamma_{2}$ ) with $m_{y} / m_{z}$ (i.e., $r / s$ ) between 0 and about 0.2 .

Lowering the temperature still further leads to the NIC phase in which both irreps $\Gamma_{2}$ and $\Gamma_{1}$ are active. The literature seems to be rather uncertain as to the actual structure of this phase. However, one possibility, seemingly not men- tioned up to now, is that application of a magnetic field to the collinear-commensurate (1/4) state could essentially give rise to a spin-flop transition so that the spins, instead of being aligned along the hexagonal $\mathbf{c}$ axis, would rotate to being nearly perpendicular to the c axis. This observation would suggest that if we ignore the lattice distortion, we would expect to have an incommensurate state with the spins elliptically polarized in a plane nearly (but not exactly) perpendicular to the hexagonal c axis. Such a state is consistent with Eq. (136) providing $\left|\phi_{2}-\phi_{1}\right|=\pi / 2$. It does have to be admitted that the spin-flop field of about $7 \mathrm{~T}$ is rather large for an $L=0$ ion such as $\mathrm{Fe}^{3+}$ whose anisotropy could be expected to be small.

So far, we have considered only two of the vectors, $\mathbf{q}_{1}$ and $-\mathbf{q}_{1}$, of the star of the wave vector. However, the Landau expansion should treat all wave vectors in the star symmetrically, since at quadratic order the system can equally well condense into any of the wave vectors of the star. So, we write the quadratic free energy $F_{2}$ as

$$
F_{2}=\sum_{n=1}^{3}\left[a_{1}(H, T)\left|\sigma_{1}\left(\mathbf{q}_{n}\right)\right|^{2}+a_{2}(H, T)\left|\sigma_{2}\left(\mathbf{q}_{n}\right)\right|^{2}\right] .
$$

When the temperature is lowered at a magnetic field of about $10 \mathrm{~T}$ along the $z$ axis, the coefficient $a_{2}(H, T)$ first passes through zero and only one of the order parameters $\sigma_{2}\left(\mathbf{q}_{n}\right)$ becomes nonzero. At lower temperature, $a_{1}(H, T)$ passes through zero and one enters a phase in which both $\sigma_{1}\left(\mathbf{q}_{n}\right)$ and $\sigma_{2}\left(\mathbf{q}_{n}\right)$ become nonzero. Within the Landau theory, it is possible to realize a phase in which two or three noncollinear wave vectors simultaneously become unstable. However, since such "double $q$ " or "triple $q$ " states are not realized for $\mathrm{CFO}$, we will not analyze this possibility further than to say that the fourth-order terms must be such as to stabilize states having a single wave vector.

The ferroelectric phase of interest is one in which $\sigma_{1}\left(\mathbf{q}_{n}\right)$ and $\sigma_{2}\left(\mathbf{q}_{n}\right)$ are nonzero for a single value of $n$. (The value of $n$ represents a broken symmetry.) For future reference, we note that at zero applied electric and magnetic fields, the free energy must be invariant under taking either $\boldsymbol{\sigma}_{1}$ or $\boldsymbol{\sigma}_{2}$ into its negative. Finally, we record how order parameters corresponding to different wave vectors of the star are related by the threefold rotation $\mathbf{3}$ :

$$
\mathbf{3} \sigma_{n}\left(\mathbf{q}_{1}\right)=\sigma_{n}\left(\mathbf{q}_{2}\right), \quad \mathbf{3}^{2} \sigma_{n}\left(\mathbf{q}_{1}\right)=\sigma_{n}\left(\mathbf{q}_{3}\right) .
$$

However, the spin distributions corresponding to these order parameters of the other wave vectors are the rotated version of the spin structure, so that if we consider the ordering wave vector $\mathbf{q}_{2}$, we have

$$
\begin{aligned}
& m_{x}(\mathbf{r})=- {\left[\sigma_{1}\left(\mathbf{q}_{2}\right) / 2\right] \cos \left(-q x / 2-q y \sqrt{3} / 2+\phi_{1}\right) } \\
&- {\left[\sqrt{3} \sigma_{2}\left(\mathbf{q}_{2}\right) r / 2\right] \cos \left(-q x-q y \sqrt{3} / 2+\phi_{2}\right), } \\
& m_{y}(\mathbf{r})=-\left[\sigma_{2}(\mathbf{q}) r / 2\right]\left(-q x / 2-q y \sqrt{3} / 2+\phi_{2}\right) \\
&+\left[\sqrt{3} \sigma_{1}\left(\mathbf{q}_{2}\right) / 2\right]\left(-q x / 2-q y \sqrt{3} / 2+\phi_{1}\right), \\
& m_{z}(\mathbf{r})=\sigma_{2}\left(\mathbf{q}_{2}\right) s \cos \left(-q x / 2-q y \sqrt{3} / 2+\phi_{2}\right) .
\end{aligned}
$$


TABLE XVIII. Generators $\mathbf{G}_{n}$ of rotational symmetry for the symmorphic space groups of RFMO. Here, $\mathcal{R}$ is a rotation through $2 \pi / 3$ about the positive $\mathbf{c}$ axis and $2_{x}$ is a twofold rotation about the a axis, as in Fig. 9.

\begin{tabular}{lccc}
\hline \hline Space group & $\mathbf{G}_{1}$ & $\mathbf{G}_{2}$ & $\mathbf{G}_{3}$ \\
\hline$P \overline{3} m 1$ & $\mathcal{R}$ & $\mathcal{I}$ & 2 \\
$P \overline{3}$ & $\mathcal{R}$ & $\mathcal{I}$ & \\
\hline \hline
\end{tabular}

To summarize, representation theory usefully restricts the possible spin structures one can obtain via one or more continuous phase transitions. Recognition of this fact might have saved a lot of experimental effort in determining the spin structures of $\mathrm{CuFeO}_{2}$.

\section{E. $\mathrm{RbFe}\left(\mathrm{MoO}_{4}\right)_{2}$}

In this section, we elaborate on a briefer presentation of the symmetry analysis given previously ${ }^{8}$ for $\mathrm{RbFe}\left(\mathrm{MoO}_{4}\right)_{2}$ (RFMO). This symmetry analysis is consistent with the microscopic model of interaction proposed by Gasparovic. ${ }^{67}$ RFMO consists of two-dimensional triangular lattice layers of Fe spin 5/2 ions (perpendicular to the crystal c axis) such that adjacent layers are stacked directly over one another. These layers of magnetic ions are separated by oxygen tetrahedra which surround a Mo ion. At room temperature the crystal structure is $P \overline{3} m 1$ (No. 164 in Ref. 33), but at $180 \mathrm{~K}$ a small lattice distortion leads to the lower symmetry $P \overline{3}$ (No. 147 in Ref. 33) structure, ${ }^{67}$ whose general lattice positions are specified in Table XVIII, and the structure is shown in Fig. 9. The low-temperature structure differs from that

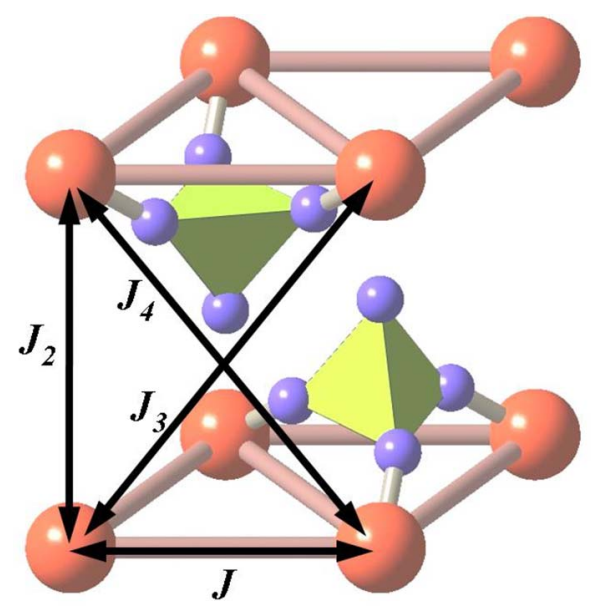

FIG. 9. (Color online) The unit cell of RFMO in the $P \overline{3}$ phase. The large balls (pink online) represent the magnetic Fe ions, the small balls (blue online) represent oxygen ions, and each tetrahedron (green online) contains a Mo ion. For clarity, the $\mathrm{Rb}$ ion (which sits between the two tetrahedra) is not shown. The in-plane antiferromagnetic interaction $J$ is dominant. In the high-temperature $P \overline{3} m 1$ phase, $J_{3}=J_{4}$, but in the presence of the lattice distortion to the $P \overline{3}$ phase, $J_{3} \neq J_{4}$ (Ref. 67 ). The a axis is parallel to the bond labeled $J$.

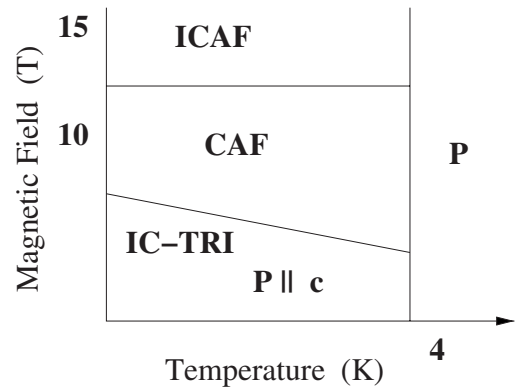

FIG. 10. A schematic phase diagram of RFMO for magnetic fields of up to about $10 \mathrm{~T}$ along the $\mathbf{c}$ axis, based on Refs. 8 and $67-70$. Here, $P$ is the paramagnetic phase and IC-TRI is an incommensurate phase described in the text in which each plane consists of the so-called $120^{\circ}$ triangular lattice structure. CAF is a commensurate antiferromagnet phase and ICAF an incommensurate antiferromagnetic phase, neither of which is discussed in the present paper. We omit reference to subtle phase distinctions discussed in Refs. 68 and 69.

above $T=180 \mathrm{~K}$ by not having the twofold rotation about the crystal a axis. As we will explain, this loss of symmetry has important consequences for the magnetic structure. ${ }^{67}$

We now discuss the magnetic structure of RFMO. A schematic magnetic phase diagram for magnetic fields of up to about $10 \mathrm{~T}$ along the $\mathbf{c}$ axis is shown in Fig. 10. The magnetic anisotropy is such that all the spins lie in the basal plane perpendicular to the c axis. The dominant interactions responsible for long-range magnetic order are antiferromagnetic interactions between nearest neighbors in a given basal plane which give rise to the so-called $120^{\circ}$ structure, shown in Fig. 11 in which the angle between all nearest neighboring spins in a basal plane is $120^{\circ} .68,69$

Here, we will be mainly interested in the properties of the phase which occurs for magnetic fields of less than about 3 T. Neutron diffraction ${ }^{8,67}$ confirms that in this phase, each triangular layer orders into a phase in which the angle between the direction of adjacent spins is $120^{\circ}$. Neutron diffraction $^{8,67}$ also indicated that from one triangular layer to

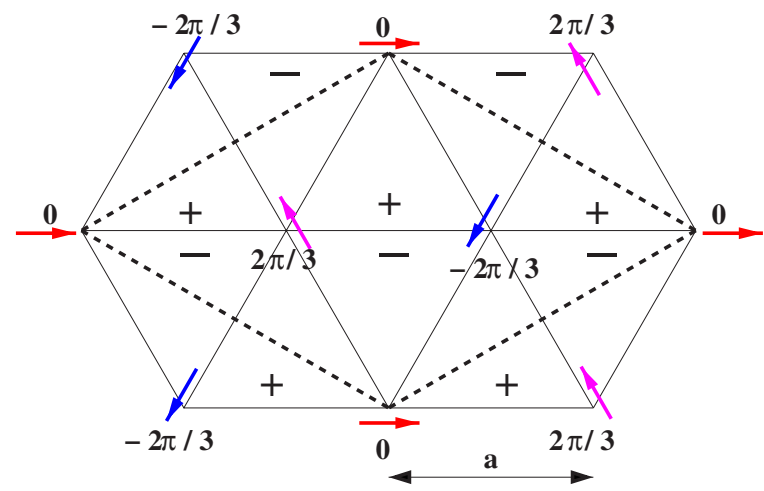

FIG. 11. (Color online) The $120^{\circ}$ phase of a triangular lattice. The orientations of the spins are given by the phase $\psi(\mathbf{r})$, defined in Eq. (157) below, for $q_{z} z+\phi=0$. The dashed lines indicate the twodimensional unit cell. The plus and minus signs indicate whether the oxygen tetrahedron closest to the center of the triangle is above (plus) or below (minus) the plane of the paper. 


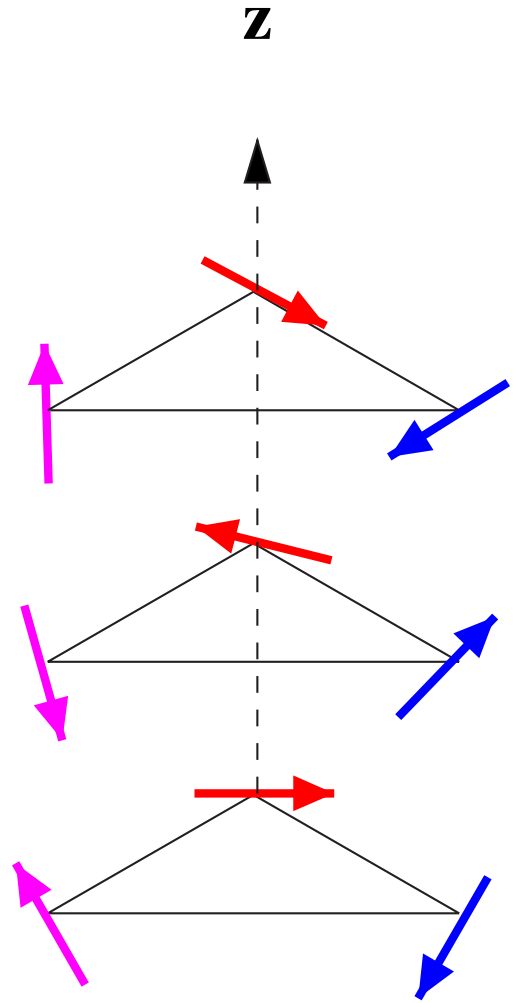

FIG. 12. (Color online) Helical spin structure of RFMO. As one moves from one triangular lattice plane to the next, the spins are rotated through an angle $166^{\circ}$ (Refs. 8 and 67).

the next, the spins are rotated through an angle $\Delta \phi=166^{\circ}, 8,67$ as shown in Fig. 12. This phase lacks inversion symmetry and is ferroelectric. ${ }^{8}$ In that reference, the order parameters which describe the magnetic ordering are discussed and we give the analysis in more detail here.

We now discuss the wave vectors which generate this magnetic structure. The $120^{\circ}$ magnetic structure of a triangular lattice is generated by wave vectors at the corners of the two-dimensional Brillouin zone, which is shown in Fig. 13. Note that the corners of the zone labeled $X_{n}$ having the same $n$ are equivalent to one another because they differ by a vector of the reciprocal lattice. However, $\mathbf{X}_{1}$ and $\mathbf{X}_{2}$, although the negatives of one another, are distinct. The incommensurate low field phase is thus characterized by the wave vectors

$$
\mathbf{Q}_{n} \equiv \mathbf{X}_{n}+q_{z} \hat{k}
$$

where the component of wave vector along $\mathbf{c}$ describes the twisting of the spins as one moves along the $\mathbf{c}$ axis via $\Delta \phi=q_{z} c$, where $c$ is the interlayer separation. It is clear that for either of the two relevant space groups, the only operation (other than the identity) that conserves wave vector is $\mathcal{R}$. The Fourier coefficients of the spin will be eigenvectors of $\mathcal{R}$ with eigenvalue $\lambda(\mathcal{R})$ and we list these in Table XIX.

The Fourier amplitude $\overline{\mathbf{S}}(\mathbf{q})$ is defined by

$$
\mathbf{S}(\mathbf{r})=\overline{\mathbf{S}}(\mathbf{q}) e^{-i \mathbf{q} \cdot \mathbf{r}}
$$

The allowed complex-valued Fourier amplitudes $\bar{S}(\mathbf{q})$ for each irrep are given in Table XIX. We now verify the results

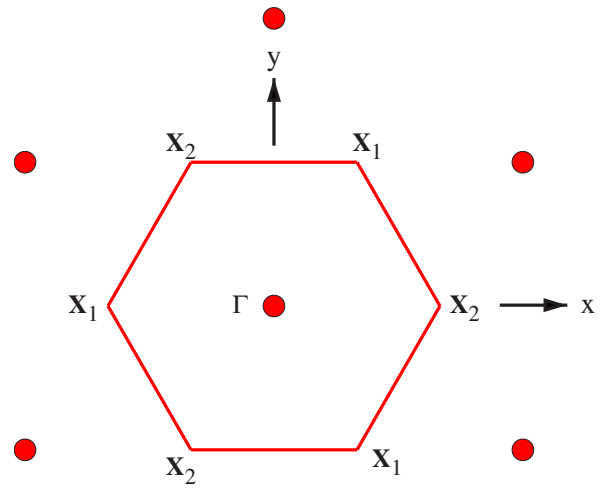

FIG. 13. (Color online) The first Brillouin zone (the hexagon) and the reciprocal lattice (the dots) for a triangular lattice. The points labeled $X_{1}$ are all equivalent and similarly for the points labeled $X_{2}$. Here, $\left|\mathbf{X}_{n}\right|=4 \pi /(\sqrt{3} a)$. The reciprocal lattice is rotated by $30^{\circ}$ with respect to the direct lattice. In reciprocal lattice units, $\mathbf{X}_{2}=(1 / 3,1 / 3,0)$.

given in Table XIX. To do this, we need to know what effect the threefold rotation $\mathcal{R}$ has on the Fourier coefficient $\overline{\mathbf{S}}(\mathbf{q})$. Let primes denote the value of quantities after transformation by $\mathcal{R}$ and unprimed quantities the quantities before transformation. We write

$$
\mathbf{S}^{\prime}\left(\mathbf{r}^{\prime}\right)=\overline{\mathbf{S}}^{\prime}(\mathbf{q}) e^{-i \mathbf{q} \cdot \mathbf{r}^{\prime}}
$$

Thus, if we can determine how $\mathbf{S}(\mathbf{r})$ and $\mathbf{r}$ transform into $\mathbf{S}^{\prime}\left(\mathbf{r}^{\prime}\right)$ and $\mathbf{r}^{\prime}$, respectively, we can use this relation to infer how $\bar{S}(\mathbf{q})$ transforms. For this discussion, we introduce the notation that $\mathcal{R}_{S}$ rotates only the spin and $\mathcal{R}_{r}$ rotates only the position, so that

$$
\mathcal{R}=\mathcal{R}_{S} \mathcal{R}_{r}
$$

Note that after transformation, the spin at $\mathbf{r}^{\prime}$ will be the rotated version of the spin that was at $\mathbf{r}$. Therefore,

$$
\mathbf{S}^{\prime}\left(\mathbf{r}^{\prime}\right)=\mathcal{R}_{S} \mathbf{S}(\mathbf{r})=\left[\mathcal{R}_{S} \overline{\mathbf{S}}(\mathbf{q})\right] e^{-i \mathbf{q} \cdot \mathbf{r}} .
$$

However,

$$
\mathbf{q} \cdot \mathbf{r}=\mathbf{q} \cdot\left[\mathcal{R}_{r}^{-1} \mathbf{r}^{\prime}\right]=\left[\mathcal{R}_{r} \mathbf{q}\right] \cdot \mathbf{r}^{\prime}=\mathbf{q} \cdot \mathbf{r}^{\prime} .
$$

Here, we used the fact that under $\mathcal{R}_{r}$ the $X$ point (see Fig. 13) goes into a point equivalent to itself. Thus,

TABLE XIX. Complex-valued Fourier components $\overline{\mathbf{S}}(\mathbf{q})$ for the various irreps. Here, $\mu=e^{2 \pi i / 3}$.

\begin{tabular}{lccc}
\hline \hline Irrep & $\Gamma_{1}$ & $\Gamma_{2}$ & $\Gamma_{3}$ \\
\hline$\lambda(\mathcal{R})$ & 1 & $\mu$ & $\mu^{2}$ \\
$S_{x}$ & 0 & $S_{\perp}$ & $S_{\perp}$ \\
$S_{y}$ & 0 & $-i S_{\perp}$ & $i S_{\perp}$ \\
$S_{z}$ & $S_{\|}$ & 0 & 0 \\
\hline \hline
\end{tabular}




$$
\mathbf{S}^{\prime}\left(\mathbf{r}^{\prime}\right)=\left[\mathcal{R}_{S} \overline{\mathbf{S}}(\mathbf{q})\right] e^{-i \mathbf{q} \cdot \mathbf{r}^{\prime}} .
$$

Comparison with Eq. (142) then yields

$$
\overline{\mathbf{S}}^{\prime}(\mathbf{q})=\mathcal{R}_{S} \bar{S}(\mathbf{q}),
$$

which we write as

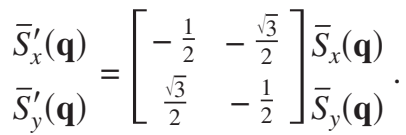

We now can check the result in Table XIX. If

$$
\overline{\mathbf{S}}(\mathbf{q})=\left(S_{\perp},-i S_{\perp}\right),
$$

then Eq. (148) gives

$$
\overline{\mathbf{S}}^{\prime}(\mathbf{q})=\mu\left(S_{\perp},-i S_{\perp}\right) \equiv \mu \overline{\mathbf{S}}(\mathbf{q}),
$$

where $\mu=\exp (2 \pi i / 3)$, as expected for $\Gamma_{2}$.

\section{Order parameters}

We now describe the spin structures corresponding to the various irreps. The distribution function for spin depends on the irrep, $\Gamma_{2}$ or $\Gamma_{3}$, on which $\mathbf{X}$ point is chosen, and on the value of the $z$ component of wave vector. So, the possible distributions are

$$
\begin{aligned}
& \mathbf{S}^{(2)}\left(\mathbf{X}_{1}, q_{z} ; \mathbf{r}\right)=R_{\perp} e^{-i\left(\mathbf{X}_{1} \cdot \mathbf{r}_{\|}+q_{z} z-\phi\right)}(\hat{i}-i \hat{j})+\text { c.c. } \\
& \mathbf{S}^{(3)}\left(\mathbf{X}_{1}, q_{z} ; \mathbf{r}\right)=R_{\perp} e^{-i\left(\mathbf{X}_{1} \cdot \mathbf{r}_{\|}+q_{z} z-\phi\right)}(\hat{i}+i \hat{j})+\text { c.c. } \\
& \mathbf{S}^{(2)}\left(\mathbf{X}_{2}, q_{z} ; \mathbf{r}\right)=R_{\perp} e^{-i\left(\mathbf{X}_{2} \cdot \mathbf{r}_{\|}+q_{z} z-\phi\right)}(\hat{i}-i \hat{j})+\text { c.c. } \\
& \mathbf{S}^{(3)}\left(\mathbf{X}_{2}, q_{z} ; \mathbf{r}\right)=R_{\perp} e^{-i\left(\mathbf{X}_{2} \cdot \mathbf{r}_{\|}+q_{z} z-\phi\right)}(\hat{i}+i \hat{j})+\text { c.c. }
\end{aligned}
$$

where the superscript on $\mathbf{S}$ labels the irrep and $\mathbf{r}_{\|}$is the in-plane part of the vector $\mathbf{r}$. Here, we have written the complex Fourier coefficient $S_{\perp}$ as $R_{\perp} \exp (-i \phi)$, where $R_{\perp}$ and $\phi$ are real. We interpret $R_{\perp} e^{-i \phi}$ as being the complex-valued order parameter $\boldsymbol{\sigma}$.

The distributions involving $\mathbf{X}_{2}$ are redundant. Since $\mathbf{X}_{2}+q_{z} \hat{k}=-\left[\mathbf{X}_{1}-q_{z} \hat{k}\right]$, one sees that

$$
\mathbf{S}^{(2)}\left(\mathbf{X}_{2}, q_{z} ; \mathbf{r} ;-\phi\right)=\mathbf{S}^{(3)}\left(\mathbf{X}_{1},-q_{z} ; \mathbf{r} ; \phi\right) .
$$

Thus, the order parameter for $\mathbf{X}_{2}$ is equivalent to the complex conjugate of that for $\mathbf{X}_{1}$ when the sign of $q_{z}$ is reversed. Accordingly, we only introduce order parameters $\sigma_{n} e^{-i \phi_{n}}$ associated with $\mathbf{X}_{1}$ by writing

$$
\begin{aligned}
& \mathbf{S}^{(2)}\left(\mathbf{X}_{1}, q_{z} ; \mathbf{r}\right)=\sigma_{2}\left(q_{z}\right) e^{-i \phi_{2}} e^{-i\left(\mathbf{X}_{1} \cdot \mathbf{r}_{\|}+q_{z} z\right)}(\hat{i}-i \hat{j})+\text { c.c. }, \\
& \mathbf{S}^{(3)}\left(\mathbf{X}_{1}, q_{z} ; \mathbf{r}\right)=\sigma_{3}\left(q_{z}\right) e^{-i \phi_{3}} e^{-i\left(\mathbf{X}_{1} \cdot \mathbf{r}_{\|}+q_{z} z\right)}(\hat{i}+i \hat{j})+\text { c.c. }
\end{aligned}
$$

The magnetic structures which these order parameters describe are best visualized in terms of the phase

$$
\psi(\mathbf{r}) \equiv \mathbf{X}_{1} \cdot \mathbf{r}_{\|}+q_{z} z+\phi .
$$

One see that for $\mathbf{S}^{(2)}$ the spin at $\mathbf{r}$ is oriented in the plane and makes angle $-\psi(\mathbf{r})$ with respect to the positive $x$ axis, whereas for $\mathbf{S}^{(3)}$ the spin at $\mathbf{r}$ is oriented in the plane and makes angle $\psi(\mathbf{r})$ with respect to the positive $x$ axis. We show the phase (for $q_{z} z+\phi=0$ ) in Fig. 11. There are some properties of the two-dimensional system which do not carry over to the three-dimensional structure. For instance, for the two-dimensional system, the plane of the lattice is a mirror plane and therefore this magnetic structure cannot possibly induce a ferroelectric moment. Also for the two-dimensional system shown, we could not distinguish between $\psi(\mathbf{r})$ and $-\psi(\mathbf{r})$ since these are related via a twofold rotation about an axis perpendicular to the plane of the lattice. Now, we discuss the relevance of Fig. 11 to RFMO. In Fig. 9, one sees that triangles have the closest oxygen tetrahedra alternatingly above and below the lattice. So, we define "positive triangles" to be those for which the oxygen tetrahedra closest to the center of the triangle are above the plane. Suppose in Fig. 11 that these are the triangles with a vertex oriented upward. We indicate these by "+" signs and the downward triangles by "-" signs. Note that if we ignored the three dimensionality (i.e., if we ignored the plus and minus signs), then we could change the sign of $\psi$ by a twofold rotation about an axis perpendicular to the lattice plane. However, since this operation interchanges + into - , it is not a symmetry of the three-dimensional lattice and the two spin distributions of Eq. (156) are distinguishable. The effect of the additional phase $\Delta \psi \equiv q_{z} z+\phi$ is to rotate all the spins in a given plane through the angle $\Delta \psi$ and thus $q_{z}$ determines the helicity. For $q_{z}>0, \mathbf{S}^{(2)}$ has negative helicity since its spin orientations follow $-\psi(\mathbf{r})$, whereas $\mathbf{S}^{(3)}$ has positive helicity since its spin orientations follow $\psi(\mathbf{r})$. The chirality of a triangle is usually defined as being positive or negative according to whether the spin rotates through plus or minus $120^{\circ}$ as one traverses the vertices of a triangle counterclockwise. In Fig. 11, the up triangles have positive chirality and the down ones negative chirality. Thus, this structure does not have overall chirality.

We now consider the symmetry of the order parameter. First of all,

$$
\begin{gathered}
\mathcal{R} \boldsymbol{\sigma}_{2}=\mu \boldsymbol{\sigma}_{2}, \\
\mathcal{R} \boldsymbol{\sigma}_{3}=\mu^{*} \boldsymbol{\sigma}_{3} .
\end{gathered}
$$

Note the effect of inversion which transports the spin to the spatially inverted location without changing its orientation. So, 


$$
\begin{aligned}
\mathcal{I} \mathbf{S}^{(2)}\left(\mathbf{X}_{1}, q_{z} ; \mathbf{r}\right) & =\mathbf{S}^{(2)}\left(\mathbf{X}_{1}, q_{z} ;-\mathbf{r}\right) \\
& =S_{\perp} e^{-i \phi} e^{i\left(\mathbf{X}_{1} \cdot \mathbf{r}_{\|}+q_{z} z\right)}(\hat{i}-i \hat{j})+\text { c.c. } \\
& =\left[S_{\perp} e^{i \phi} e^{-i\left(\mathbf{X}_{1} \cdot \mathbf{r}_{\|}+q_{z} z\right)}(\hat{i}+i \hat{j})\right]^{*}+\text { c.c. } \\
& =S_{\perp} e^{i \phi} e^{-i\left(\mathbf{X}_{1} \cdot \mathbf{r}_{\|}+q_{z} z\right)}(\hat{i}+i \hat{j})+\text { c.c. }
\end{aligned}
$$

This relation is equivalent to saying that

$$
\mathcal{I} \boldsymbol{\sigma}_{2}\left(q_{z}\right)=\boldsymbol{\sigma}_{3}\left(q_{z}\right)^{*} .
$$

The symmetry operation $2_{x}$ only holds in the hightemperature $(P \overline{3} m 1)$ phase. For it,

$$
{ }_{x} \mathbf{S}^{(2)}\left(\mathbf{X}_{1}, q_{z} ; \mathbf{r}\right)=\boldsymbol{\sigma}_{2}\left(q_{z}\right) e^{-i\left(\mathbf{X}_{1} \cdot \mathbf{r}_{\|}-q_{z} z\right)}(\hat{i}+i \hat{j}),
$$

so that

$$
2_{x} \boldsymbol{\sigma}_{2}\left(q_{z}\right)=\boldsymbol{\sigma}_{3}\left(-q_{z}\right)^{*}
$$

Now, the quadratic free energy (keeping terms involving both irreps and both signs of $q_{z}$ ) is of the form

$$
F_{2}=A\left|\sigma_{2}\left(q_{z}\right)\right|^{2}+B\left|\sigma_{3}\left(q_{z}\right)\right|^{2}+C\left|\sigma_{2}\left(-q_{z}\right)\right|^{2}+D\left|\sigma_{3}\left(-q_{z}\right)\right|^{2} .
$$

A continuous phase transition occurs at a temperature at which one or more of the coefficients $A, B, C$, and $D$ become zero. Using Eq. (160), we see that inversion symmetry ensures that $A=B$ and $C=D$. In the high-temperature phase, $2_{x}$ symmetry ensures that $A=D$ and $B=C$. Thus, wave vector selection in the high-temperature phase would not select the sign of $q_{z}$. Indeed, if, as is believed, the dominant interplanar interactions are antiferromagnetic interactions between nearest neighbors in adjacent layers $\left(J_{2}\right.$ in Fig. 9), then had there been no lattice distortion at $180 \mathrm{~K}$, one would select $q_{z}$ $=1 / 2$ (which is equivalent to $q_{z}=-1 / 2$ ). Since the $2_{x}$ symmetry is lost below $180 \mathrm{~K}$, in that range of temperature we should write $A-C=B-D=c^{\prime} \eta$, where $\eta$ is an order parameter describing the amplitude of the lattice distortion and $c^{\prime}$ is a constant whose sign can be related to the quantity $J_{3}$ $-J_{4} \cdot{ }^{67}$ Accordingly, we write the free energy relative to the high-temperature undistorted paramagnetic phase in terms of the structural $(\eta)$ and magnetic $(\boldsymbol{\sigma}$ 's) order parameters as

$$
\begin{aligned}
F_{2}= & A\left(T-T_{D}\right) \eta^{2}+u \eta^{4}+\sum_{q_{z}>0} \sum_{n=2}^{3}\left\{\left[\alpha\left(T-T_{c}\right)+J_{\mathrm{av}} \cos \left(q_{z} c\right)\right]\right. \\
& \times\left[\left|\boldsymbol{\sigma}_{n}\left(q_{z}\right)\right|^{2}+\left|\boldsymbol{\sigma}_{n}\left(-q_{z}\right)\right|^{2}\right]-c^{\prime} \eta \sin \left(q_{z} c\right)\left[\left|\boldsymbol{\sigma}_{n}\left(q_{z}\right)\right|^{2}\right. \\
& \left.\left.-\left|\boldsymbol{\sigma}_{n}\left(-q_{z}\right)\right|^{2}\right]\right\}+\mathcal{O}\left(\sigma^{4}\right),
\end{aligned}
$$

where $T_{D}=180 \mathrm{~K}$ is the temperature at which the lattice distortion appears, $T_{c}$ is the mean-field transition temperature for $120^{\circ}$ magnetic ordering on the triangular lattice, and $J_{\mathrm{av}}$ represents the sum of the interplanar antiferromagnetic interactions that do not select the sign of $q_{z}$. Also, we have included the results of a microscopic model ${ }^{67}$ in which the term in $c^{\prime}$ comes from distortion-modified interactions which give the term proportional to $c^{\prime} \sin \left(q_{z} c\right)$, which leads to the lifting of degeneracy between $+q_{z}$ and $-q_{z}$ when $\eta \neq 0$.

So, the situation is the following. When we cool through $T_{D} \equiv 180 \mathrm{~K}$, the system arbitrarily breaks crystal symmetry from $P \overline{3} m 1$ and rotates the oxygen tetrahedra into the $P \overline{3}$ structure. ${ }^{67}$ Here, the angle of rotation can have either sign, depending on the sign of the broken symmetry order parameter $\eta$. For the sake of argument, say that $\eta$ is positive. Now, when the temperature is lowered so that magnetic ordering takes place, ordering takes place in the channels $\boldsymbol{\sigma}_{2}\left(q_{z}\right)$ and/or $\boldsymbol{\sigma}_{3}\left(q_{z}\right)$, where $q_{z}$ is the value of $q_{z}$ at which an instability with respect to $\boldsymbol{\sigma}$ first appears as the temperature is lowered. At quadratic order, the phases $\phi_{n}$ of the order parameters $\sigma_{n}\left(q_{z}\right)$ are arbitrary and also the relative proportion of each irrep is not fixed. However, it is expected that the fourth-order terms in the Landau expansion (which tend to enforce fixed spin length) will favor having only a single irrep present. So, ordering is expected either in $\boldsymbol{\sigma}_{2}$ or in $\boldsymbol{\sigma}_{3}$, but we can have domains of both, in addition to possibly having domains of either sign of $\eta$. Although the domains of different $\boldsymbol{\sigma}$ 's have the same wave vector, they have opposite helicity, as discussed just above Eq. (158).

\section{F. Discussion}

\section{Summary of results}

In Table XX, we collect the results for various multiferroics.

\section{Effect of quartic terms}

As we now discuss, the quartic terms in the Landau expansion can have significant qualitative effects. ${ }^{6}$ In general, the quartic terms are the lowest-order ones which favor the fixed length spin constraint, a constraint which is known to be dominant at low temperature. ${ }^{72}$ How this constraint comes into play depends on what state is selected by the quadratic terms. For instance, in the simplest scenario when one has a ferromagnet or an antiferromagnet, the instability is such (see Fig. 1) that ordering with uniform spin length takes place. Thus, as the temperature is lowered within the ordered phase, the ordering of wave vectors near $q=0$ for the ferromagnet (near $q=\pi$ for the antiferromagnet), which would have become unstable if only the quadratic terms were relevant, is strongly disfavored by the quartic terms. In the systems considered here, the situation is quite different. For instance, in $\mathrm{NVO},{ }^{38} \mathrm{TMO},{ }^{3}$ and $\mathrm{MWO},{ }^{45}$ the quadratic terms select an incommensurate structure in which the spins are aligned along an easy axis and their magnitudes are sinusoidally modulated. As the temperature is lowered, the quartic terms lead to an instability in which transverse spin component breaks the symmetry of the longitudinal incommensurate phase. This scenario explains why the highesttemperature incommensurate longitudinal phase becomes unstable to a lower-temperature incommensurate phase which has both longitudinal and transverse components which more nearly conserve spin length.

To see this result formally for NVO, TMO, or MWO, let $\boldsymbol{\sigma}_{>}\left(\boldsymbol{\sigma}_{<}\right)$be the complex-valued order parameter for the higher-temperature longitudinal (lower-temperature transverse) ordering. The fourth-order terms then lead to the free energy as 
TABLE XX. Incommensurate phases of various multiferroics. Except for CFO, each phase is stable for zero applied magnetic field for $T_{<}<T<T_{>}$. When $T_{<}=0$, it means that the phase is stable down to the lowest temperature investigated. We give the incommensurate wave vector and the associated irreducible representations in the notation of our tables. In the column labeled "FE?" if the system is ferroelectric we give the direction of the spontaneous polarization, otherwise the entry is "No."

\begin{tabular}{|c|c|c|c|c|c|c|c|}
\hline Phase & $T_{<}(\mathrm{K})$ & $T_{>}(\mathrm{K})$ & $\mathbf{q}$ & Irreps & Ref. & FE? & Ref. \\
\hline NVO (HTI) & 6.3 & 9.1 & $(\mathrm{q}, 0,0)$ & $\Gamma_{4}$ & 6 and 38 & No & 4 and 6 \\
\hline NVO (LTI) & 3.9 & 6.3 & $(\mathrm{q}, 0,0)$ & $\Gamma_{4}+\Gamma_{1}$ & 6 and 38 & $\| b$ & 4 and 6 \\
\hline TMO (HTI) & 28 & 41 & $(0, q, 0)$ & $\Gamma_{3}$ & 3 and 49 & No & 2 \\
\hline TMO (LTI) & & 28 & $(0, q, 0)$ & $\Gamma_{3}+\Gamma_{2}$ & 3 & $\| c$ & 2 \\
\hline $\mathrm{TbMn}_{2} \mathrm{O}_{5}(\mathrm{HTI})$ & 38 & 43 & $\left(\frac{1}{2}, 0, q\right)^{\mathrm{a}}$ & $\Gamma^{\mathrm{b}}$ & 55 and 56 & No & 12 \\
\hline $\mathrm{TbMn}_{2} \mathrm{O}_{5}(\mathrm{LTI})$ & 33 & 38 & $\left(\frac{1}{2}, 0, q\right)$ & $\Gamma^{\mathrm{c}}$ & 55 and 56 & $\| b$ & 12 \\
\hline $\mathrm{YMn}_{2} \mathrm{O}_{5}(\mathrm{C})^{\mathrm{d}}$ & 23 & 45 & $\left(\frac{1}{2}, 0, \frac{1}{4}\right)$ & $\Gamma^{\mathrm{b}}$ & 58 & $\| b$ & 12 \\
\hline $\mathrm{YMn}_{2} \mathrm{O}_{5}(\mathrm{IC})$ & & 23 & $\left(\approx \frac{1}{2}, 0, q\right)$ & & 58 & $\| b$ & 12 \\
\hline $\mathrm{RFMO}^{\mathrm{e}}$ & 0 & 3.8 & $\left(\frac{1}{3}, \frac{1}{3}, q\right)$ & $\Gamma_{2}$ or $\Gamma_{3}$ & 8 and 67 & $\| c$ & 8 \\
\hline $\mathrm{CFO}^{\mathrm{f}}(\mathrm{CIC})$ & 10 & 14 & $(q, q, 0)$ & $\Gamma_{2}$ & 60 and 66 & No & 10 \\
\hline CFO (NIC) & $0 ?$ & 10 & $(q, q, 0)$ & $\Gamma_{1}+\Gamma_{2}$ & 61 & $\perp c$ & 10 \\
\hline MWO & 12.7 & 13.2 & $\left(q_{x}, \frac{1}{2}, q_{z}\right)$ & $\Gamma_{2}$ & 45 & No & 13 \\
\hline MWO & 7.6 & 12.7 & $\left(q_{x}, \frac{1}{2}, q_{z}\right)$ & $\Gamma_{2}+\Gamma_{1}$ & 45 & $\| b$ & 13 \\
\hline
\end{tabular}

${ }^{a}$ At the highest temperature, the value of $q_{x}$ might not be exactly $1 / 2$.

${ }^{b}$ The irrep is the two-dimensional one (see Appendix B). In the HTI phase, only one basis vector is active.

${ }^{\mathrm{c}}$ The irrep is the two-dimensional one (see Appendix B). In the LTI phase, both basis vectors are active.

${ }^{\mathrm{d}}$ This phase is commensurate.

e For $H<2 T$.

${ }^{\mathrm{f}}$ Data for $\mathrm{CuFeO}_{2}$ are for $H \approx 8 \mathrm{~T}$.

$$
\begin{aligned}
F= & a\left(T-T_{>}\right)\left|\boldsymbol{\sigma}_{>}\right|^{2}+b\left(T-T_{<}\right)\left|\boldsymbol{\sigma}_{<}\right|^{2}+A\left(\left|\boldsymbol{\sigma}_{>}\right|^{2}+\left|\boldsymbol{\sigma}_{<}\right|^{2}\right)^{2} \\
& +B\left|\boldsymbol{\sigma}_{>} \boldsymbol{\sigma}_{<}\right|^{2}+C\left[\left(\boldsymbol{\sigma}_{<} \boldsymbol{\sigma}_{>}^{*}\right)^{2}+\left(\boldsymbol{\sigma}_{<}^{*} \boldsymbol{\sigma}_{>}\right)^{2}\right],
\end{aligned}
$$

where $A, B$, and $C$ are real. That $C$ is real is a result of inversion symmetry, which, for these systems, leads to $\mathcal{I} \boldsymbol{\sigma}_{n}$ $=\boldsymbol{\sigma}_{n}^{*}$. The high-temperature representation does allow transverse components and could, in principle, satisfy the fixed length constraint. In the usual situation, however, the exchange couplings are nearly isotropic and this state is not energetically favored. If the higher-temperature structure is longitudinal, then $B$ will surely be negative, whereas if the higher-temperature structure conserves spin length, $B$ will probably be positive. By properly choosing the relative phases of the two order parameters, the term in $C$ always favors having two irreps. So, the usual scenario in which the longitudinal phase becomes unstable relative to transverse ordering is explained (in this phenomenology) by having $B$ be negative, so that the discussion after Eq. (51) applies.

To finish the argument, it remains to consider the term in $C$, which can be written as

$$
\delta F_{4}=2 C \sigma_{<}^{2} \sigma_{>}^{2} \cos \left(2 \phi_{<}-2 \phi_{>}\right),
$$

where again we expressed the order parameters as in Eq. (46). Normally, if two irreps are favored, it is because together they better satisfy the fixed length constraint. What that means is that when spins have substantial length in one irrep, the contribution to their spin length from the second irrep is small. In other words, the two irreps are out of phase and we therefore expect that to minimize $\delta F_{4}$, we do not set $\phi_{<}=\phi_{>}$, but rather

$$
\phi_{<}=\phi_{>} \pm \pi / 2 .
$$

In other words, we expect $C$ in Eq. (166) to be positive. The same reasoning indicates that the fourth-order terms will favor $\phi_{2}-\phi_{1}=\pi / 2$ in Eq. (136) for CFO.

For all of these systems which have two consecutive continuous transitions, one has a family of broken symmetry states. At the highest-temperature transition, one has spontaneously broken symmetry which arbitrarily selects between $\sigma_{>}$and $-\sigma_{>}$. (This is the simplest scenario when the wave vector is not truly incommensurate.) Independent of which sign is selected for the order parameter $\boldsymbol{\sigma}_{>}$, one similarly has a further spontaneous breaking of symmetry to obtain arbitrarily either $i \sigma_{<}$or $-i \sigma_{<}$. (Here, as mentioned, we assume a relative phase $\pi / 2$ for $\boldsymbol{\sigma}_{<}$.) In this scenario, then, there are four equivalent low-temperature phases corresponding to the choice of signs of the two order parameters.

The cases of TMO25 and YMO25 are different from the above because they have two order parameters from the same two-dimensional irrep and which therefore are simultaneously critical. Therefore, in such a case we write 


$$
\begin{aligned}
F= & a\left(T-T_{c}\right)\left[\left|\boldsymbol{\sigma}_{1}\right|^{2}+\left|\boldsymbol{\sigma}_{2}\right|^{2}\right]+A\left(\left|\boldsymbol{\sigma}_{1}\right|^{2}+\left|\boldsymbol{\sigma}_{2}\right|^{2}\right)^{2}+B\left|\boldsymbol{\sigma}_{1} \boldsymbol{\sigma}_{2}\right|^{2} \\
& +C\left[\left(\boldsymbol{\sigma}_{1} \boldsymbol{\sigma}_{2}^{*}\right)^{2}+\left(\boldsymbol{\sigma}_{1}^{*} \boldsymbol{\sigma}_{2}\right)^{2}\right] .
\end{aligned}
$$

Here, again $A, B$, and $C$ are real. That $C$ is real is a result of symmetry under $m_{y}$, as in Eq. (124). Here, the fourth-order anisotropy makes itself felt as soon as the ordered phase is entered, but the above discussion about the sign of $B$ remains operative. We first consider YMO25 in its highertemperature commensurate (HTC) ordered phase. For it, additional fourth-order terms occur because $4 \mathbf{q}$ is a reciprocal lattice vector, but these are not important for the present discussion. Here, the analysis of Ref. 58 indicates (see the discussion of our Fig. 7) that only a single order parameter condenses in the HTC phase. This indicates that energetics must favor positive $B$ in this case. The question is whether $B$ is also positive for TMO25. As we will see in the next section, one has ferroelectricity unless the magnitudes of the two order parameters are the same. For YMO25, the HTC phase is ferroelectric and the conclusion that only one order parameter is active comports with this. However, for TMO25 the situation is not completely clear. Apparently, there is a region such that one has magnetic ordering without ferroelectricity. ${ }^{55,12}$ If this is so, then TMO25 differs from YMO25 in that its high-temperature incommensurate phase has two equal magnitude order parameters.

\section{MAGNETOELECTRIC COUPLING}

Ferroelectricity is induced in these incommensurate magnets by a coupling which is somewhat similar to that for the so-called "improper ferroelectrics." ${ }^{17}$ To see how such a coupling arises within a phenomenological picture, we imagine expanding the free energy in powers of the magnetic order parameters, which we have studied in detail in the previous section, and also the vector order parameter for ferroelectricity, which is the spontaneous polarization $\mathbf{P}$ which, of course, is a zero wave vector quantity. If we had noninteracting magnetic and electric systems, then we would write the noninteracting free energy $F_{\text {non }}$ as

$$
\begin{aligned}
F_{\text {non }}= & \frac{1}{2} \sum_{\alpha} \chi_{E, \alpha}^{-1} P_{\alpha}^{2}+\mathcal{O}\left(P^{4}\right)+\frac{1}{2} \sum_{\Gamma} a_{\Gamma}\left(T-T_{\Gamma}\right)\left|\sigma_{\Gamma}(\mathbf{q})\right|^{2} \\
& +\mathcal{O}\left(\sigma^{4}\right),
\end{aligned}
$$

where $\chi_{E, \alpha}^{-1}$ is of order unity. The first line describes a system which is not close to being unstable relative to developing a spontaneous polarization (since in the systems we consider ferroelectricity is induced by magnetic ordering). The magnetic terms describe the possibility of having one or more phase transitions at which successively more magnetic order parameters become nonzero. As we have mentioned, the scenario of having two phase transitions in incommensurate magnets is a very common one,${ }^{30}$ and such a scenario is well documented for both $\mathrm{NVO}^{6,38}$ and TMO., ${ }^{2,3}$ Below, we will indicate the existence of a term linear in $P$, schematically of the form $-\lambda M^{2} P$, where $\lambda$ is a coupling constant about which not much beyond its symmetry is known. One sees that when the free energy, including this term, is minimized with respect to $P$, one obtains the equilibrium value of $P$ as

$$
\langle P\rangle=\chi_{E} \lambda M^{2} .
$$

\section{A. Symmetry of magnetoelectric interaction}

We now consider the free energy of the combined magnetic and electric degrees of freedom, which we write as

$$
F=F_{\text {non }}+F_{\text {int }} .
$$

In view of time reversal invariance and wave vector conservation, the lowest combination of $M(q)$ 's that can appear is proportional to $M_{\alpha}(-\mathbf{q}) M_{\beta}(\mathbf{q})$. So, generically the term we focus on will be of the form

$$
F_{\text {int }}=\sum_{\alpha \beta \gamma} c_{\alpha \beta \gamma} M_{\alpha}(\mathbf{q}) M_{\beta}(-\mathbf{q}) P_{\gamma},
$$

where $\alpha, \beta$, and $\gamma$ label Cartesian components. However, as we have seen in detail, the quantities $M_{\alpha}(\mathbf{q})$ are linearly related to the order parameter $\sigma_{\Gamma}(\mathbf{q})$, associated with the irrep $\Gamma$. Thus, instead of Eq. (172), we write

$$
F_{\text {int }}=\sum_{\Gamma, \Gamma^{\prime}, \gamma} A_{\Gamma \Gamma^{\prime} \gamma} \sigma_{\Gamma}(\mathbf{q}) \sigma_{\Gamma^{\prime}}(\mathbf{q})^{*} P_{\gamma} .
$$

The advantage of writing interaction in this form is that it is expressed in terms of quantities whose symmetry is manifest. In particular, the order parameters we have introduced have well specified symmetries. For instance, it is easy to see that for most of the systems studied here, magnetism cannot induce ferroelectricity when there is only a single representation present. ${ }^{3,4}$ This follows from the fact that for NVO and $\mathrm{TMO}$, for instance,

$$
\mathcal{I}\left|\sigma_{n}\right|^{2}=\left|\sigma_{n}\right|^{2},
$$

as is evident from Eq. (50). The interpretation of this is simple: When one has one representation, it is essentially the same as having a single incommensurate wave. However, such a single wave will have inversion symmetry (to as close a tolerance as we wish) with respect to some lattice point. This is enough to exclude ferroelectricity. So, the canonical scenario $^{3,4}$ is that ferroelectricity appears not when the first incommensurate magnetic order parameter condenses, but rather when a second such order parameter condenses. Unless the two waves have the same origin, their centers of inversion symmetry do not coincide and there is no inversion symmetry and hence ferroelectricity will occur. One might ask whether or not the two waves (i.e., two irreps) will be in phase. The effect, discussed above, of quartic terms is crucial here. The quartic terms typically favor the fixed length spin constraint. To approximately satisfy this constraint, one needs to superpose two waves which are out of phase. Indeed, the formal result, obtained below in Eq. (179), shows that the spontaneous polarization is proportional to the sine of the phase difference between the two irreps. ${ }^{4}$ We now consider the various systems in turn.

\section{B. NVO, TMO, and MWO}

We now analyze the canonical magnetoelectric interaction in the cases of NVO, TMO, ad MWO. These cases are all 
similar to one another and in each case the order parameters have been defined so as to obey Eq. (50). This relation indicates that if we are in a phase for which only one irrep is active, then we may choose the origin of the incommensurate system so that the phase of the order parameter at the origin of a unit cell is arbitrarily close to zero. When this phase is zero, the spin distribution of this irrep has inversion symmetry relative to this origin. In the case when only a single irrep is active, this symmetry then indicates that the magnetic structure cannot induce a spontaneous polarization. ${ }^{4}$ As mentioned, in the high-temperature incommensurate phases of NVO, TMO, and MWO, only one irrep is present, and this argument indicates that the magnetoelectric interaction vanishes, in agreement with the experimental observation ${ }^{2,4,13}$ that this phase is not ferroelectric. Notice that this argument relies on symmetry and does not invoke the fact that the HTI phase may involve a collinear spin structure (as it seems for TMO and MWO, but not for NVO). Small departures from collinearity (induced by, say, Dzialoshinskii-Moriya interactions ${ }^{73}$ ) do not change the symmetry of the structure and therefore cannot induce ferroelectricity. This conclusion is not obvious from the spin-current models. ${ }^{15,16}$

We now turn to the general case when one or more irreps are present. ${ }^{4-7}$ We write the magnetoelectric interaction as

$$
F_{\text {int }}=\sum_{\gamma \Gamma \Gamma^{\prime}} A_{\Gamma \Gamma^{\prime} \gamma} \boldsymbol{\sigma}_{\Gamma}(\mathbf{q}) \boldsymbol{\sigma}_{\Gamma^{\prime}}(\mathbf{q})^{*} P_{\gamma}
$$

where $\boldsymbol{\sigma}_{\Gamma}(\mathbf{q})=\boldsymbol{\sigma}_{\Gamma}(-\mathbf{q})^{*}$. For this to yield a real value of $F$, we must have Hermiticity:

$$
A_{\Gamma \Gamma^{\prime} \gamma}=A_{\Gamma^{\prime} \Gamma \gamma^{\prime}}^{*}
$$

In addition, because this is an expansion relative to the state in which all order parameters are zero, this interaction has to be inversion under all operations which leave this "vacuum" state invariant. ${ }^{26,31}$ In other words, this interaction has to be invariant under inversion (which takes $P_{\gamma}$ into $-P_{\gamma}$ ). In view of Eq. (50), we conclude that $A_{\Gamma, \Gamma^{\prime}, \gamma}$ vanishes for $\Gamma^{\prime}=\Gamma$. Thus, for these systems, it is essential to have the simultaneous existence of two distinct irreps. A similar phenomenological description of second harmonic generation has also invoked the necessity of simultaneously having two irreps. ${ }^{71}$ (We will see below that systems such as TMO25, YMO25, and RFMO provide exceptions to this statement.) So, we write

$$
F_{\text {int }}=\frac{1}{2} \sum_{\gamma \Gamma \Gamma^{\prime}: \Gamma \neq \Gamma^{\prime}} A_{\Gamma \Gamma^{\prime} \gamma} \boldsymbol{\sigma}_{\Gamma}(\mathbf{q}) \boldsymbol{\sigma}_{\Gamma^{\prime}}(\mathbf{q})^{*} P_{\gamma}
$$

Now, invoke Eq. (50). Since inversion changes the sign of $P_{\gamma}$, we conclude that $A_{\Gamma \Gamma^{\prime} \gamma}=-A_{\Gamma^{\prime} \Gamma \gamma}$. This condition taken in conjunction with Eq. (176) indicates that $A_{\Gamma \Gamma^{\prime} \gamma}$ is purely imaginary. Thus,

$$
F_{\mathrm{int}}=\frac{i}{2} \sum_{\gamma \Gamma \Gamma^{\prime}: \Gamma<\Gamma^{\prime}} P_{\gamma} r_{\Gamma \Gamma^{\prime} \gamma}\left[\boldsymbol{\sigma}_{\Gamma}(\mathbf{q}) \boldsymbol{\sigma}_{\Gamma^{\prime}}(\mathbf{q})^{*}-\boldsymbol{\sigma}_{\Gamma}(\mathbf{q})^{*} \boldsymbol{\sigma}_{\Gamma^{\prime}}(\mathbf{q})\right],
$$

where $r_{\Gamma \Gamma^{\prime} \gamma}$ is real valued. Since usually we have at most two different irreps, which we label " $>$ " and " $<$ " we write this as

$$
F_{\text {int }}=\sum_{\gamma} r_{\gamma} P_{\gamma} \sigma_{>} \sigma_{<} \sin \left(\phi_{>}-\phi_{<}\right) .
$$

where $r_{\gamma}$ is real and $\boldsymbol{\sigma}_{<}=\sigma_{<} \exp \left(i \phi_{<}\right)$and similarly for the irrep $>$. The fact that the result vanishes when the two waves are in phase is clear because in that case one can find a common origin for both irreps about which one has inversion symmetry. In that special case, one has inversion symmetry and no spontaneous polarization can be induced by magnetism. The above argument applies to all three systems, NVO $,{ }^{4} \mathrm{TMO},{ }^{3}$ and MWO. As we will see in a moment, it is still possible for inversion symmetry to be broken and yet induced ferroelectricity not be allowed.

We can also deduce the direction of the spontaneous polarization by using the transformation properties of the order parameters given in Eq. (49). We start by analyzing the experimentally relevant cases at low or zero applied magnetic field. For NVO, the magnetism in the lower-temperature incommensurate phase is described ${ }^{6,38}$ by the two irreps $\Gamma_{4}$ and $\Gamma_{1}$. One sees from Eq. (49) that the product $\sigma_{1}^{*} \sigma_{4}$ is even under $m_{z}$ and odd under $2_{x}$. For the interaction to be an invariant, $P_{\gamma}$ has to transform this way also. This implies that only the $\mathbf{b}$ component of the spontaneous polarization can be nonzero, as observed. ${ }^{4}$ For TMO, the lower-temperature incommensurate phase at low magnetic field is described ${ }^{3}$ by irreps $\Gamma_{3}$ and $\Gamma_{2}$. In Table XII, we see that $\sigma_{3}^{*} \sigma_{2}$ is even under $m_{x}$ and odd under $m_{z}$, which indicates that $\mathbf{P}$ has to be even under $m_{x}$ and odd under $m_{z}$. This can only happen if $\mathbf{P}$ lies along the $\mathbf{c}$ direction, as observed. ${ }^{2}$

Finally, for MWO, we see that $\boldsymbol{\sigma}_{1} \boldsymbol{\sigma}_{2}^{*}$ is odd under $m_{y}$. This indicates that $P_{\gamma}$ also has to be odd under $m_{y}$. In other words, $\mathbf{P}$ can only be oriented along the $\mathbf{b}$ direction, again as observed. ${ }^{13}$ In this connection, one should note that this conclusion is a result of crystal symmetry, assuming that the magnetic structure results from two continuous transitions, so that representation theory is relevant. This conclusion is at variance with the argument given by Heyer et al. ${ }^{14}$ who "expect a polarization in the plane spanned by the easy axis and the $\mathbf{b}$ axis...," which they justify on the basis of the spiral model. ${ }^{15,16}$ It should be noted that their observation that the spontaneous polarization has a nonzero component along the a axis at zero applied magnetic field contradicts the symmetry analysis given here. The authors mention that some of the unexpected behavior they observe might possibly be attributed to a small content of impurities.

It is important to realize that the above results are a consequence of crystal symmetry. In view of that, it is not sensible to claim that the fact that a theory gives the result that the polarization lies along $\mathbf{b}$ makes it more plausible than some competing theory. The point is that any model, if analyzed correctly, must give the correct orientation for $\mathbf{P}$. 
It is also worth noting that this phenomenology has some semiquantitative predictions. To see this, we minimize $F_{\text {non }}$ $+F_{\text {int }}$ with respect to $\mathbf{P}$ to get

$$
P_{\gamma}=-\chi_{E, \gamma} r_{\gamma} \sigma_{>} \sigma_{<} \sin \left(\phi_{>}-\phi_{<}\right) .
$$

This result indicates that near the magnetoferroelectric phase transition of NVO, one has $P \propto \sigma_{4} \sigma_{1},{ }^{74}$ or since the hightemperature order parameter $\sigma_{4}$ is more or less saturated when the ferroelectric phase is entered, one has $P \propto \sigma_{1}$, where $\boldsymbol{\sigma}_{1}$ is the order parameter of the lower-temperature incommensurate phase. This relation has not been tested for NVO, TMO, or MWO, but we will see that such a relation has been observed for RFMO.

As we discussed, in the low-temperature incommensurate phase, one will have arbitrary signs of the two order parameters. However, the presence of a small electric field will favor one particular sign of the polarization and hence, by Eq. (180), one particular sign for the product $\sigma_{>} \sigma_{<}$. Presumably, this could be tested by a neutron diffraction experiment.

\section{TMO25 and YMO25}

\section{TMO25}

The case of TMO25 is somewhat different. Here, we have only a single irrep. One expects that as the temperature is lowered, ordering into an incommensurate state will take place, but the quadratic terms in the free energy do not select a direction in $\boldsymbol{\sigma}_{1}-\boldsymbol{\sigma}_{2}$ space. At present, the data have not been analyzed to say which direction is favored at temperature just below the highest ordering temperature. As the temperature is reduced, it is not possible for another representation to appear because only one irrep is involved. However, ordering according to a second eigenvalue could occur. We first analyze the situation assuming that we have only a single doubly degenerate eigenvalue. In this case, we can have a spin distribution [as given in Eq. (123)] involving the two order parameters $\boldsymbol{\sigma}_{1}$ and $\boldsymbol{\sigma}_{2}$ which measure the amplitude and phase of the ordering of the eigenvector of the second and third columns of Table XVI, respectively. In terms of these order parameters, the magnetoelectric coupling can be written as

$$
F_{\text {int }}=\sum_{n m \gamma} a_{n m \gamma} \sigma_{n}^{*} \sigma_{m} P_{\gamma}
$$

where $\gamma=x, y, z$ and $n, m=1,2$ label the columns of the irrep labeled $\boldsymbol{\sigma}_{1}$ and $\boldsymbol{\sigma}_{2}$, respectively, in Table XVI. Since reality requires that $a_{n m \gamma}=a_{m n \gamma}^{*}$, this interaction is of the form

$F_{\text {int }}=\sum_{\gamma} P_{\gamma}\left[a_{1 \gamma}\left|\boldsymbol{\sigma}_{1}\right|^{2}+a_{2 \gamma}\left|\boldsymbol{\sigma}_{2}\right|^{2}+b_{\gamma} \boldsymbol{\sigma}_{1} \boldsymbol{\sigma}_{2}^{*}+b_{\gamma}^{*} \boldsymbol{\sigma}_{1}^{*} \boldsymbol{\sigma}_{2}\right]$.

Now, use invariance under inversion, taking note of Eq. (124). One sees that under inversion, $\boldsymbol{\sigma}_{1} \boldsymbol{\sigma}_{2}^{*} P_{\gamma}$ changes sign, so the only terms which survive lead to the result

$$
F_{\text {int }}=\sum_{\gamma} r_{\gamma} P_{\gamma}\left[\left|\boldsymbol{\sigma}_{1}\right|^{2}-\left|\boldsymbol{\sigma}_{2}\right|^{2}\right] .
$$

Using Eq. (124), we see that $\left[\left|\boldsymbol{\sigma}_{1}\right|^{2}-\left|\boldsymbol{\sigma}_{2}\right|^{2}\right]$ is even under $m_{x}$ and odd under $m_{y}$. For $F_{\text {int }}$ to be invariant under inversion

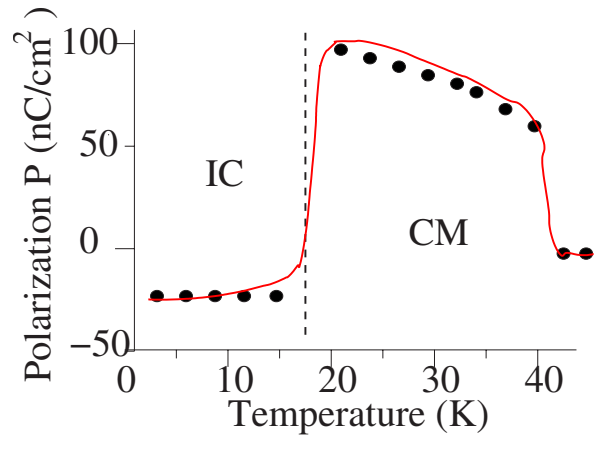

FIG. 14. (Color online) After Ref. 58: the thin line is the spontaneous polarization along the $\mathbf{b}$ axis from Ref. 12 and the filled circles are from a microscopic model of Ref. 58 described in the text. The dashed line indicates the commensurate to incommensurate phase transition at about $23 \mathrm{~K}$.

therefore requires that $P_{\gamma}$ be odd under $m_{y}$ and even under $m_{x}$, so $\mathbf{P}$ has to be along $\mathbf{b}$ as is found. ${ }^{12}$

\section{YMO25}

For YMO25, as mentioned above, in the highesttemperature commensurate phase, the magnetic structure is such that $\boldsymbol{\sigma}_{1}=0$. Thus, in this phase, we have a spontaneous polarization which is proportional to the square of the order parameter, so that $P_{b} \propto\left|\sigma_{2}\right|^{2}$ and this seems consistent with the data shown in Fig. 14. In that figure, we also show the result of a microscopic model developed in Ref. 58, which is based on a microscopic trilinear interaction of a strain with two spin operators, as could emerge from a spin-phonon interaction. ${ }^{75}$ The agreement between the calculation and the data is impressive.

At the commensurate to incommensurate first-order transition at about $23 \mathrm{~K}$, the spins in the unit cell are reoriented. It is not easy to obtain the order parameters of the lowtemperature incommensurate phase from Ref. 58. However, if we normalize the order parameters to that $\sigma_{2}=1$ just above the transition at $23 \mathrm{~K}$, then we obtain the estimate that $-0.25<\left|\sigma_{2}\right|^{2}-\left|\sigma_{1}\right|^{2}<0.25$. Thus, the order-parameter analysis is consistent with the sharp decrease in the magnitude of the polarization below the phase transition. It should be obvious that the phenomenological interaction of Eq. (183) and the microscopic interaction of Ref. 58 are closely related and must, in fact, have the same symmetry. Very recently, Betouras et $a l .{ }^{76}$ have proposed an alternate interaction to partially explain these data. However, their interaction does not have the correct symmetry properties to match with the microscopic calculation and also their model gives $P=0$ in the low-temperature phase. ${ }^{59}$

\section{CFO}

Again, we start with the trilinear magnetoelectric interaction, but here we have to allow for coupling of the spontaneous polarization to order parameters associated with any of the wave vectors in the star. So, we write

$$
F_{\text {int }}=\sum_{k n m \gamma} A_{n m k \gamma} \boldsymbol{\sigma}_{n}\left(\mathbf{q}_{k}\right) \boldsymbol{\sigma}_{m}\left(\mathbf{q}_{k}\right)^{*} P_{\gamma},
$$

where $k$ is summed over the values 1,2 , and 3 and reality implies that $A_{n m k}=A_{m n k \gamma}^{*}$. Since we have that 
$\mathcal{I} \boldsymbol{\sigma}_{n}\left(\mathbf{q}_{k}\right)=\boldsymbol{\sigma}_{n}\left(\mathbf{q}_{k}\right)^{*}$, we use invariance under $\mathcal{I}$ to eliminate terms with $n=m$ : We need two irreps for ferroelectricity. Indeed, the higher-temperature phase with a single order parameter $\boldsymbol{\sigma}_{2}$ is not ferroelectric. ${ }^{10}$ Thus, the magnetoelectric interaction must be of the form

$$
F_{\mathrm{int}}=\sum_{k \gamma}\left[A_{k \gamma} \boldsymbol{\sigma}_{1}\left(\mathbf{q}_{k}\right) \boldsymbol{\sigma}_{2}\left(\mathbf{q}_{k}\right)^{*}+A_{k \gamma}^{*} \boldsymbol{\sigma}_{1}\left(\mathbf{q}_{k}\right)^{*} \boldsymbol{\sigma}_{2}\left(\mathbf{q}_{k}\right)\right] P_{\gamma} .
$$

Inversion symmetry indicates that $A_{k \gamma}=-A_{k \gamma}^{*}$, so we write

$$
\begin{aligned}
F_{\text {int }} & =i \sum_{k \gamma} r_{k \gamma}\left[\boldsymbol{\sigma}_{1}\left(\mathbf{q}_{k}\right) \boldsymbol{\sigma}_{2}\left(\mathbf{q}_{k}\right)^{*}-\boldsymbol{\sigma}_{1}\left(\mathbf{q}_{k}\right)^{*} \boldsymbol{\sigma}_{2}\left(\mathbf{q}_{k}\right)\right] P_{\gamma} \\
& =2 \sum_{k \gamma} r_{k \gamma} \sigma_{1}\left(\mathbf{q}_{k}\right) \sigma_{2}\left(\mathbf{q}_{k}\right) \sin \left(\phi_{2}-\phi_{1}\right) P_{\gamma},
\end{aligned}
$$

where $r_{k \gamma}$ is real. Now, consider the term involving wave vector $\mathbf{q}_{1}$ and use Eq. (135) which gives that $\boldsymbol{\sigma}_{1}\left(\mathbf{q}_{1}\right) \boldsymbol{\sigma}_{2}\left(\mathbf{q}_{1}\right)^{*}$ changes sign under $2_{x}$. So, for the interaction to be invariant under $2_{x}$ (as it must be), $P_{\gamma}$ has to be odd under $2_{x}$. This means that for $\mathbf{q}=\mathbf{q}_{1}, \mathbf{P}$ has to be perpendicular to the $x$ axis. So,

$$
\begin{aligned}
F_{\text {int }}= & 2 \sigma_{1}\left(\mathbf{q}_{1}\right) \sigma_{2}\left(\mathbf{q}_{1}\right) \sin \left(\phi_{2}-\phi_{1}\right)\left[a P_{z}+b P_{y}\right] \\
& +2 \sigma_{1}\left(\mathbf{q}_{2}\right) \sigma_{2}\left(\mathbf{q}_{2}\right) \sin \left(\phi_{2}-\phi_{1}\right)\left[a P_{z}-(b / 2) P_{y}\right. \\
& \left.-(\sqrt{3} b / 2) P_{x}\right]+2 \sigma_{1}\left(\mathbf{q}_{3}\right) \sigma_{2}\left(\mathbf{q}_{3}\right) \sin \left(\phi_{2}-\phi_{1}\right)\left[a P_{z}\right. \\
& \left.-(b / 2) P_{y}+(\sqrt{3} b / 2) P_{x}\right],
\end{aligned}
$$

where the real-valued coefficients $a$ and $b$ are not fixed by symmetry. Here, we constructed the terms involving $\mathbf{q}_{2}$ and $\mathbf{q}_{3}$ by using the transformation properties of the threefold rotation, so that $F_{\text {int }}$ is invariant under all the symmetry operations. Note that symmetry does not force $\mathbf{P}$ to lie along the threefold axis because the orientation of the incommensurate wave vector has broken the threefold symmetry.

In fact, the above results suggest some further experiments. First of all, it would be useful to have a definitive determination of the spin structure of the NIC phase, in particular, to test whether our idea of a spin-flop-type transition has occurred. One should note that symmetry does not completely restrict the orientation of $\mathbf{P}$ when, for instance, the wave vector is $\mathbf{q}=\mathbf{q}_{1}$. In this connection, it is interesting to note that in Ref. 10, a component of $\mathbf{P}$ along $\mathbf{c}$ was discarded as being due to sample misalignment. However, such a component is allowed by symmetry. Although the spin-current model $1^{15,16}$ is satisfied by having the spin-flop state we suggest, our analysis indicates that this spin configuration cannot be uniquely identified just from the orientation of $\mathbf{P}$, so a determination of the actual spin structure is important. Furthermore, the form of Eq. (187) indicates that the orientation of $\mathbf{q}_{n}$ is coupled to the applied electric field in the plane perpendicular to c. In other words, by applying an electric field perpendicular to the $\mathbf{c}$ axis, one could select between the three equivalent wave vectors of the star. (Since the crystal structure distortion also implies such a selection, one would have to apply a strong enough electric field so that the electric energy overcomes the energy of the lattice distortion.)
In the above analysis, we did not mention the fact that the existence of the ferroelectric phase requires a magnetic field of about 8-10 $\mathrm{T}$ oriented along the threefold axis. In principle, one should expand the free energy in powers of $H$. Then, presumably as a function of $H$, one reaches a regime where, first, one incommensurate phase orders and then at a lower temperature the second incommensurate order parameter appears. Then, the phenomenology of the trilinear magnetoelectric interaction would come into play as analyzed above.

\section{E. RFMO}

Again we start from Eq. (175), which for the present case of two irreps $(n=2,3)$ we write

$$
F_{\text {int }}=\sum_{\gamma}\left[r_{2 \gamma}\left|\boldsymbol{\sigma}_{2}\right|^{2}+r_{3 \gamma}\left|\boldsymbol{\sigma}_{3}\right|^{2}+b_{\gamma} \boldsymbol{\sigma}_{2} \boldsymbol{\sigma}_{3}^{*}+b_{\gamma}^{*} \boldsymbol{\sigma}_{3} \boldsymbol{\sigma}_{2}^{*}\right] P_{\gamma},
$$

where $b_{\gamma}$ is complex and $r_{n \gamma}$ is real. First, use inversion symmetry under which $P_{c}$ changes sign and Eq. (160) holds. This symmetry indicates that $b_{\gamma}=0$ and $r_{2 \gamma}=-r_{3, \gamma}$, so that

$$
F_{\text {int }}=\sum_{\gamma} r_{\gamma}\left[\left|\boldsymbol{\sigma}_{2}\right|^{2}-\left|\boldsymbol{\sigma}_{3}\right|^{2}\right] P_{\gamma} .
$$

Now, consider invariance under the threefold rotation, which leaves $\left|\boldsymbol{\sigma}_{n}\right|^{2}$ invariant. One sees that the only nonzero component of $\mathbf{P}$ can be the $c$ component, so that finally

$$
F_{\text {int }}=r\left[\left|\boldsymbol{\sigma}_{2}\right|^{2}-\left|\boldsymbol{\sigma}_{3}\right|^{2}\right] P_{c} .
$$

As mentioned above, when the total free energy is minimized with respect to $P_{c}$ in order to determine its equilibrium value, one finds that

$$
P_{c}=-r \chi_{E, c}\left[\left|\boldsymbol{\sigma}_{2}\right|^{2}-\left|\boldsymbol{\sigma}_{3}\right|^{2}\right] .
$$

Since the magnetic structure RFMO has been determined ${ }^{8}$ to have only a single order parameter (call it $\boldsymbol{\sigma}_{a}$ ) in the low field phase, in this phase

$$
P_{c} \propto\left|\boldsymbol{\sigma}_{a}\right|^{2} .
$$

Since the right-hand side of this equation is proportional to the intensity of the Bragg reflections which appear as one enters the incommensurate phase, this relation predicts that these Bragg intensities are proportional to the magnitude of the spontaneous polarization. This relation has been experimentally confirmed. ${ }^{8}$

It is interesting to note that for this case, the "spiral model" or spin-current model does not apply in their simplest form. The spin rotated in a plane perpendicular to the threefold axis, so that $\mathbf{S}_{i} \times \mathbf{S}_{j}$ is parallel to the threefold axis, no matter what values $i$ and $j$ may take. In the spin-current model, the spontaneous polarization is supposed to be perpendicular to this cross product, which would incorrectly predict the spontaneous polarization to be perpendicular to the threefold axis, In contrast, experiment shows the spontaneous polarization to lie along the threefold axis. 


\section{F. High magnetic field}

We can also say a word or two about what happens when a magnetic field is applied. In TMO, for instance, one finds ${ }^{2}$ that for applied magnetic fields above about $10 \mathrm{~T}$ in either the $\mathbf{a}$ or $\mathbf{b}$ direction, the lower-temperature incommensurate phase has a spontaneous polarization along the a axis. Keep in mind that we want to identify this phase with two irreps and from the phase diagram we know that the highertemperature incommensurate phase is maintained into this high field regime. So, the higher-temperature phase is still that of $\Gamma_{3}$ at these high fields. Referring to Table XII, we see that to get $\sigma_{m} \sigma_{n}^{*}$ to be odd under $m_{x}$ and even under $m_{z}$ (in order to get a polarization along the a axis), we can only combine irrep $\Gamma_{1}$ with the assumed preexisting $\Gamma_{3}$. Therefore, it is clear that the magnetic structure has to change at the same time that direction of spontaneous polarization changes as a function of applied magnetic field. ${ }^{7,16}$ It is also interesting, in this connection, to speculate on what happens if the lower additional irrep had been $\Gamma_{4}$ so that $\Gamma_{4}$ and $\Gamma_{3}$ would coexist. In that case, $\sigma_{4} \sigma_{3}^{*}$ is odd under both $m_{x}$ and $m_{z}$. These conditions are not consistent with any direction of polarization, so in this hypothetical case, even though we have two irreps and break inversion symmetry, a polar vector (such as the spontaneous polarization) is not allowed. ${ }^{77}$

For MWO, a magnetic field along the $\mathbf{b}$ axis of about $10 \mathrm{~T}$ causes the spontaneous polarization to switch its direction from along the $\mathbf{b}$ axis to along the a axis. ${ }^{13}$ We have no phenomenological explanation of this behavior at present. This behavior seems to imply that the wave vector for $H$ $>10 \mathrm{~T}$ is no longer of the form $\mathbf{q}=\left(q_{x}, \frac{1}{2}, q_{z}\right)$.

\section{G. Discussion}

What is to be learned from the symmetry analysis of the magnetoelectric interactions? Perhaps the most important point to keep in mind is to recognize which results are purely a result of crystal symmetry and which are model dependent. For instance, as we have seen, the direction of the spontaneous polarization is usually a result of crystal symmetry. So, the fact that a microscopic theory leads to the observed direction of the polarization does not lend credence to one model as opposed to another. In a semiquantitative vein, one can say that symmetry alone predicts that near the combined magnetoelectric phase transition, $P$ will be approximately proportional to the order parameter raised to the $n$th power, where the value of $n$ is a result of symmetry $(n=1$ for NVO or TMO, whereas $n=2$ for TMO25 or RFMO).

We should also note that while the spontaneous polarization does arise from the coupling to another (magnetic) order parameter, this coupling still induces a divergence in the electric susceptibility (and hence in the dielectric constant) at the magnetoelectric phase transition. To illustrate this, we consider the less trivial case where one has two order parameters. Thus, for example, we analyze the case of NVO and consider the magnetoelectric free energy at a temperature just above the lower-temperature transition, denoted $T_{<}$, where $\sigma_{<}$develops. There, the relevant terms in the free energy are

$$
\begin{aligned}
F= & \frac{1}{2} \chi_{E, y}^{-1} P_{y}^{2}+\frac{1}{2}\left(T-T_{<}\right)\left|\boldsymbol{\sigma}_{<}\right|^{2}+\frac{1}{2}\left(T-T_{>}\right)\left|\boldsymbol{\sigma}_{>}\right|^{2}+\frac{1}{4} u\left|\boldsymbol{\sigma}_{>}\right|^{4} \\
& +\frac{i}{2} \lambda\left[\boldsymbol{\sigma}_{>} \boldsymbol{\sigma}_{<}^{*}-\boldsymbol{\sigma}_{>}^{*} \boldsymbol{\sigma}_{<}\right] P_{y}-E_{y} P_{y},
\end{aligned}
$$

where $E_{y}$ is the component of the electric field in the $y$ direction, and as before $\boldsymbol{\sigma}_{<}=\sigma_{<} e^{i \phi_{<}}$and $\boldsymbol{\sigma}_{>}=\sigma_{>} e^{i \phi_{>}}$, where, for simplicity, we have omitted the wave vector arguments. Since the magnetoelectric interaction term proportional to $\lambda$ is a small perturbation, and since the temperature is significantly less than $T_{>}$, the value of $\left|\boldsymbol{\sigma}_{>}\right|$is essentially fixed by minimizing the terms in the first line of Eq. (193) with respect to $\boldsymbol{\sigma}_{>}$. The phase of this complex order parameter is probably locked by some small commensuration energy (not written in the above equation) to a commensurate value. So, we will consider that $\boldsymbol{\sigma}_{>}$in the last line of Eq. (193) is fixed by the terms in the free energy relevant to the ordering at $T_{>}$. With this understanding, we write the free energy as

$$
\begin{aligned}
F= & \frac{1}{2} \chi_{E, y}^{-1} P_{y}^{2}+\frac{1}{2}\left(T-T_{<}\right)\left|\boldsymbol{\sigma}_{<}\right|^{2}+\frac{i}{2} \lambda\left[\boldsymbol{\sigma}_{>} \boldsymbol{\sigma}_{<}^{*}-\boldsymbol{\sigma}_{>}^{*} \boldsymbol{\sigma}_{<}\right] P_{y} \\
& -E_{y} P_{y},
\end{aligned}
$$

and we now analyze the transition at $T=T_{<}$according to this free energy. Apart from the term proportional to $E_{y}$, this free energy is a quadratic form in the variables $\boldsymbol{\sigma}_{<}$and $P_{y}$ (remember that here $\boldsymbol{\sigma}_{>}$is simply a complex constant). To diagonalize this quadratic form, it is simplest to write $\boldsymbol{\sigma}_{<}=s$ $+i t$, where $s$ and $t$ are real, and similarly we set $\boldsymbol{\sigma}_{>}=a+i b$. Then, the terms quadratic in $s, t$, and $P_{y}$ are

$$
F_{2}=\frac{1}{2} \chi_{E, y}^{-1} P_{y}^{2}+\frac{1}{2}\left(T-T_{<}\right)\left[s^{2}+t^{2}\right]+\lambda[s b-t a] P_{y} .
$$

As a preliminary to diagonalizing this form, we set

$$
\begin{aligned}
& x=[s a+t b] / \sqrt{a^{2}+b^{2}}, \\
& y=[s b-t a] / \sqrt{a^{2}+b^{2}},
\end{aligned}
$$

in which case

$$
F_{2}=\frac{1}{2} \chi_{E, y}^{-1} P_{y}^{2}+\frac{1}{2}\left(T-T_{<}\right)\left[x^{2}+y^{2}\right]+\lambda^{\prime} y P_{y},
$$

where $\lambda^{\prime}=\lambda\left|\boldsymbol{\sigma}_{>}\right|$. This form shows that the variable $x$ is decoupled from the other variables, $y$ and $P_{y}$. The normal coordinates $\tilde{y}$ and $\widetilde{P}_{y}$ are obtained from $y$ and $P_{y}$ by a transformation which eliminates the perturbative coupling $\lambda^{\prime} y P_{y}$. The transition temperature for $\tilde{y}$ is obtained explicitly below in Eq. (202) as

$$
\tilde{T}_{<}=T_{<}+\lambda^{\prime 2} \chi_{E, y} .
$$

Thus, we see that as the temperature is lowered, the variable $x$ would become critical at $T=T_{<}$, except for the fact that $\tilde{y}$ condenses first (at the higher temperature $\widetilde{T}_{<}$). To understand the meaning of the variables $x$ and $y$, write 


$$
\begin{gathered}
x=\frac{\boldsymbol{\sigma}_{<} \boldsymbol{\sigma}_{>}^{*}+\boldsymbol{\sigma}_{<}^{*} \boldsymbol{\sigma}_{>}}{2\left|\boldsymbol{\sigma}_{>}\right|}, \\
y=\frac{i\left(\boldsymbol{\sigma}_{<} \boldsymbol{\sigma}_{>}^{*}-\boldsymbol{\sigma}_{<}^{*} \boldsymbol{\sigma}_{>}\right)}{2\left|\boldsymbol{\sigma}_{>}\right|} .
\end{gathered}
$$

Thus, we see that $x$ is the part of $\boldsymbol{\sigma}_{<}$which is in phase with $\boldsymbol{\sigma}_{>}$and $y$ is the part of $\boldsymbol{\sigma}_{<}$which is out of phase with $\boldsymbol{\sigma}_{>}$. These results are completely consistent with Eq. (179).

Now, we develop an expression for the electric and magnetoelectric susceptibilities in the presence of the magnetoelectric interaction as the temperature is lowered toward the phase transition at $T \approx T_{<}$. Note that the free energy is of the form

$$
F=\frac{1}{2} \widetilde{\mathbf{v}} \mathbf{M v}-\widetilde{\mathbf{v}} \mathbf{E},
$$

where $\mathbf{v}$ the column vector with entries $P_{y}$ and $y, \mathbf{E}$ is a column vector with entries $E_{y}$ and 0 , and $\mathbf{M}$ is the matrix of coefficients of the quadratic form in $P_{y}$ and $y$ of Eq. (197). Minimization with respect to $\mathbf{v}$ yields the equation of state

$$
\mathbf{v} \equiv\left[\begin{array}{c}
P_{y} \\
y
\end{array}\right]=\mathbf{M}^{-1}\left[\begin{array}{c}
E_{y} \\
0
\end{array}\right] \text {. }
$$

Then, the renormalized electric susceptibility $\hat{\chi}_{y}$ is given by

$$
\begin{aligned}
\hat{\chi}_{y} & \left.\equiv \frac{\partial P_{y}}{\partial E_{y}}\right|_{E_{y}=0}=\frac{M_{22}}{M_{11} M_{22}-M_{12}^{2}}=\frac{\chi_{E, y}\left(T-T_{<}\right)}{\left(T-T_{<}\right)-\lambda^{\prime 2} \chi_{E, y}} \\
& \equiv \frac{\chi_{E, y}\left(T-T_{<}\right)}{\left(T-\tilde{T}_{<}\right)},
\end{aligned}
$$

so that as $T \rightarrow \widetilde{T}_{<}$, one has

$$
\hat{\chi}_{y}=\frac{\chi_{E, y}^{2} \lambda^{\prime 2}}{\left(T-\tilde{T}_{<}\right)} .
$$

Thus, the electric susceptibility diverges at $T=\widetilde{T}_{<}$(although with a severely reduced amplitude.) It can also be shown for $T$ approaching $\tilde{T}_{<}$from below that

$$
\left.\hat{\chi}_{y} \equiv \frac{\partial P_{y}}{\partial E_{y}}\right|_{E_{y}=0}=\frac{a \chi_{E, y}^{2} \lambda^{\prime 2}}{\left|T-\tilde{T}_{<}\right|},
$$

where $a$ is a constant of order unity. The magnetoelectric coupling increases the electric susceptibility even far above $T_{<}$, where

$$
\hat{\chi}_{y} \approx \chi_{E, y}\left[1+\frac{\lambda^{\prime 2} \chi_{E, y}}{T-T_{<}}\right] .
$$

The magnetoelectric susceptibility

$$
\left.\chi_{E, \sigma} \equiv \frac{\partial y}{\partial E_{y}}\right|_{E_{y}=0}
$$

gives the dependence of the magnetic order parameter $\boldsymbol{\sigma}_{<}$on the electric field. Using Eq. (201), we have

$$
\chi_{E, \sigma}=-\frac{M_{21}}{M_{11} M_{22}-M_{12}^{2}}=-\frac{\lambda^{\prime} \chi_{E, y}}{T-\widetilde{T}_{<}} .
$$

To measure this susceptibility would seem to require measuring (probably via a neutron diffraction experiment) $y$, the component of the order parameter $\sigma_{<}$which is out of phase with $\sigma_{>}$in a small electric field.

It goes without saying that our phenomenological results are supposed to apply generally, independent of what microscopic mechanism might be operative for the system in question. (A number of such microscopic calculations have appeared recently. ${ }^{15,75,78-80}$ ) Therefore, we treat YMO25 and NVO with the same methodology although these systems are said $^{55}$ to have different microscopic mechanisms. A popular phenomenological description is that given by Mostovoy ${ }^{16}$ based on a continuum formulation. However, this development, although appealing in its simplicity, does not correctly capture the symmetry of several systems because it completely ignores the effect of the different possible symmetries within the magnetic unit cell. ${ }^{77}$ Furthermore, it does not apply to multiferroic systems, such as YMO25 or RFMO, in which the plane of rotation of the spins is perpendicular to the wave vector. ${ }^{8,58}$ (The spin-current model ${ }^{15}$ also does not explain ferroelectricity in these systems.) In addition, a big advantage of the symmetry analysis presented here concerns small perturbations. While the structure of NVO and TMO is predominantly a spiral in the ferroelectric phase, one can speculate on whether there are small spiral-like components in the nonferroelectric (HTI) phase. In other words, could small transverse components lead to a small (maybe too small for current experiments to see) spontaneous polarization? If we take into account the small magnetic moments induced on the oxygen ions, could these lead to a small spontaneous polarization in an otherwise nonferroelectric phase? The answer to these questions is obvious within a symmetry analysis like that we have given: These induced effects are still governed by the symmetry of the phase which can only be lowered by a spontaneous symmetry breaking (which we only expect if we cross a phase boundary). Therefore, all such possible induced effects are taken into account by our symmetry analysis.

Finally, we note that the form of the magnetoelectric interaction $\sim M^{2} P$ suggests a microscopic mechanism that has general validity, although it is not necessarily the dominant mechanism. This observation stimulated an investigation of the spin-phonon interaction one obtains by considering the exchange Hamiltonian

$$
\mathcal{H}=\sum_{i j \alpha \beta} J_{\alpha \beta}(i, j) S_{\alpha}(i) S_{\beta}(j) .
$$

When $J_{\alpha \beta}(i, j)$ is expanded to linear order in phonon displacements $u$, one obtains a magnetoelectric interaction of the form $u S S .^{75}$ After some algebra, it was shown ${ }^{75}$ that the results for the direction of the induced spontaneous polarization (when the spins are ordered appropriately) agree with the results of the symmetry arguments used here. In addition, a first-principles calculation of the phonon modes ${ }^{75}$ led to plausible guesses as to which phonon modes play the key 
TABLE XXI. Irreducible representations of the paramagnetic space group of NVO. The vector representations are $B_{1 u}, B_{2 u}$, and $B_{3 u}$, whose wave functions transform like $z, y$, and $x$, respectively.

\begin{tabular}{lccccccccc}
\hline \hline & 1 & $2_{y}$ & $2_{x}$ & $2_{z}$ & $\mathcal{I}$ & $m_{y}$ & $m_{x}$ & $m_{z}$ & Function \\
\hline$A_{g}$ & 1 & 1 & 1 & 1 & 1 & 1 & 1 & 1 & $x^{2}, y^{2}, z^{2}$ \\
$A_{u}$ & 1 & 1 & 1 & 1 & -1 & -1 & -1 & -1 & $x y z$ \\
$B_{2 g}$ & 1 & 1 & -1 & -1 & 1 & 1 & -1 & -1 & $x z$ \\
$B_{2 u}$ & 1 & 1 & -1 & -1 & -1 & -1 & 1 & 1 & $y$ \\
$B_{3 g}$ & 1 & -1 & 1 & -1 & 1 & -1 & 1 & -1 & $y z$ \\
$B_{3 u}$ & 1 & -1 & 1 & -1 & -1 & 1 & -1 & 1 & $x$ \\
$B_{1 g}$ & 1 & -1 & -1 & 1 & 1 & -1 & -1 & 1 & $x y$ \\
$B_{1 u}$ & 1 & -1 & -1 & 1 & -1 & 1 & 1 & -1 & $z$ \\
\hline \hline
\end{tabular}

role in the magnetoelectric coupling, but whatever the microscopic model, the phenomenology presented here should apply.

\section{DYNAMICS}

Here, we briefly indicate how symmetry considerations apply to dynamical properties. We consider two phenomena, namely, (a) the mixing of the infrared active phonons with the Raman active phonons when inversion symmetry is broken and (b) the mixing of electric dipole allowed transitions into spin resonance transitions which previously were only magnetic dipole allowed.

\section{A. Phonon mixing}

We discuss phonon dynamics with respect to coordinates appropriate to the phase which is paramagnetic and paraelectric. In that phase, at zero wave vector, the phonon modes can be classified as even (Raman active) or odd (infrared active). Here, we display explicitly the interaction which causes the mixing of even and odd modes when the ferroelectric phase (for which inversion symmetry is broken) is entered. In the ferroelectric phase, the spontaneous dipole moment is induced by the trilinear magnetoelectric interaction discussed above in detail. Here, we discuss the mixing of even and odd modes for NVO, since NVO has been the object of detailed phonon calculations. ${ }^{75}$ As discussed in that reference, the existence of a nonzero spontaneous dipole moment along the crystal $\mathbf{b}$ axis (which here we call the $y$ axis) reflects the fact that all the zone-center phonon modes which transform like the $y$ component of a vector develop nonzero static displacements. We now consider the anharmonic phonon interactions. (The present discussion is more detailed than that of Valdes Aguilar et al., ${ }^{81}$ but is otherwise identical to what they have done.) In particular, the third-order interactions can be written as

$$
\begin{aligned}
V^{(3)}= & \sum_{\mathbf{q}_{1} \mathbf{q}_{2} \mathbf{q}_{3}} \sum_{\alpha \beta \gamma} c_{\alpha \beta \gamma}\left(\mathbf{q}_{1} \mathbf{q}_{2} \mathbf{q}_{3}\right) Q_{\alpha}\left(\mathbf{q}_{1}\right) Q_{\beta}\left(\mathbf{q}_{2}\right) Q_{\gamma}\left(\mathbf{q}_{3}\right) \\
& \times \Delta\left(\mathbf{q}_{1}+\mathbf{q}_{2}+\mathbf{q}_{3}\right),
\end{aligned}
$$

where $Q_{\alpha}(\mathbf{q})$ is the amplitude of the $\alpha$ th phonon at wave vector $\mathbf{q}$ and $\Delta$ is only nonzero when its argument is zero modulo a reciprocal lattice vector. The terms in this interaction which are relevant to our discussion are those which mix even and odd modes at zero wave vector. So, we set all the wave vectors to zero in Eq. (209). In addition, since we want to discuss how modes mix, we write the effective bilinear interaction as

$$
V^{(3)}=\sum_{\alpha \beta \gamma} c_{\alpha \beta \gamma} Q_{\alpha}(0) Q_{\beta}(0)\left\langle Q_{\gamma}(0)\right\rangle,
$$

where \langle\rangle indicates a static average value. Because the interaction only involves zero wave vector modes, we can profitably use their symmetry properties. Accordingly, in Table XXI, we record the symmetries of the various phonon modes. To emphasize the symmetry of the modes, we label the modes as $Q_{\Gamma}^{(n)}$, where $\Gamma$ is the irreducible representation (irrep), which we identify by its function ( $y$ for $B_{2 u}, x y z$ for $A_{u}$, etc., and 1 for $A_{g}$ ). Only the $B_{2 u}$ modes which transform like $y$ can have a nonzero average value because, as we have seen, in NVO the spontaneous polarization is fixed by symmetry to lie along the $y$ axis. The interaction of Eq. (210) has to be invariant under the symmetry operations of the para phase. Therefore, the interaction can only contain the following terms:

$$
\begin{aligned}
V^{(3)}= & \sum_{n}\left\langle Q_{y}^{(n)}\right\rangle\left[a_{n m r} Q_{1}^{(m)} Q_{y}^{(r)}+b_{n m r} Q_{x y z}^{(m)} Q_{x z}^{(r)}+c_{n m r} Q_{y z}^{(m)} Q_{z}^{(r)}\right. \\
& \left.+d_{n m r} Q_{x y}^{(m)} Q_{x}^{(r)}\right] .
\end{aligned}
$$

This interaction mixes odd symmetry modes which initially were only infrared active (except for $x y z$ modes which are silent) into modes which were previously only Raman active (transforming like 1, $x z, y z$, or $x y$ ). Similarly, this interaction mixes even symmetry modes which initially were only Raman active into modes which were previously only infrared active (transforming like $x, y$, or $z$ ). Experiments can distinguish the polarization dependence of the infrared and Raman modes, so one can test the prediction that modes which were, for example, $x y$-like Raman modes are now infrared active under $x$-polarized radiation. Since the admixture in the wave function is proportional to $\left\langle Q_{y}^{(n)}\right\rangle$, which itself is proportional to the spontaneous polarization, one sees that the new intensities are scaled by the square of the spontaneous polarization. Also, in the presence of a weak perturbation, the mode 


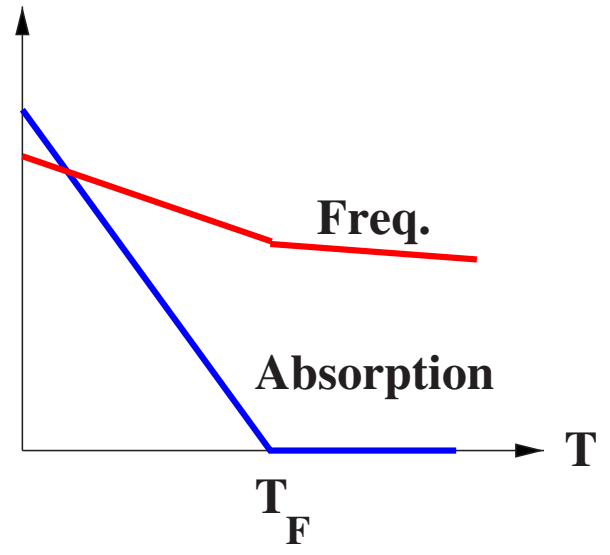

FIG. 15. (Color online) Schematic diagram of the frequency and infrared absorption cross section of a mode which is Raman active in the paraelectric phase for $T>T_{F}$. Note the change in slope of the frequency when the ferroelectric phase is entered. We assume the mean-field estimate for the order parameter: $P \propto\left(T_{F}-T\right)^{1 / 2}$.

energies will show an additional temperature dependence (in addition to what they had in the paraelectric phase) which is also proportional to the square of the spontaneous polarization. This is illustrated schematically in Fig. 15.

\section{B. Electromagnons}

Here, I give a brief discussion of "electromagnons." This term refers to the possibility of exciting magnons through an electric dipole matrix element. ${ }^{82-85}$ The existence of this process implies a mixing of spin operators and the spontaneous polarization, so that the spin wave develops a dipole moment. In general terms, such an interaction is implied by the trilinear magnetoelectric interaction studied in Sec. IV. The treatment here includes elements from the theories of Katsura et al. ${ }^{83}$ and of Pimenov et al. ${ }^{82,84}$

Again, to exemplify the idea, I describe the situation for NVO (the case of TMO is almost identical) and will focus on the HTI phase where only the single order parameter $\boldsymbol{\sigma}_{\mathrm{HTI}}$ of irrep $\Gamma_{4}$ is nonzero. The aim of the present discussion is to analyze the constraints of symmetry on the equations of motion. ${ }^{83}$ Since it is only in the HTI phase that symmetry provides constraints on the electromagnon interaction, ${ }^{82,84}$ we concentrate on this case, without assuming a specific model of interactions.

We start by writing the equation of motion for the Green's function for an infrared active phonon in the notation of Zubarev, ${ }^{86}$

$$
\omega^{2}\left\langle\left\langle Q_{\alpha, m} ; Q_{\alpha, m}\right\rangle\right\rangle=1+\left\langle\left\langle\partial \mathcal{H} / \partial Q_{\alpha, m} ; Q_{\alpha, m}\right\rangle\right\rangle
$$

where $Q_{\alpha, m}$ is the $m$ th mass weighted normal coordinate for the zero wave vector of $\alpha$-like symmetry $(\alpha=x, y, z) \cdot{ }^{75}$ In the absence of the magnetoelectric interaction, we set $\partial \mathcal{H} / \partial Q_{\alpha, m}=\omega_{\alpha, m}^{2} Q_{\alpha, m}$. We now include the magnetoelectric interaction $V_{\mathrm{e}-\mathrm{m}}$. In the HTI phase of NVO where only the order parameter $\boldsymbol{\sigma}_{>}$of irrep $\Gamma_{4}$ is present, the spin-phonon coupling we need to mix modes must arise from an effective bilinear interaction of the form
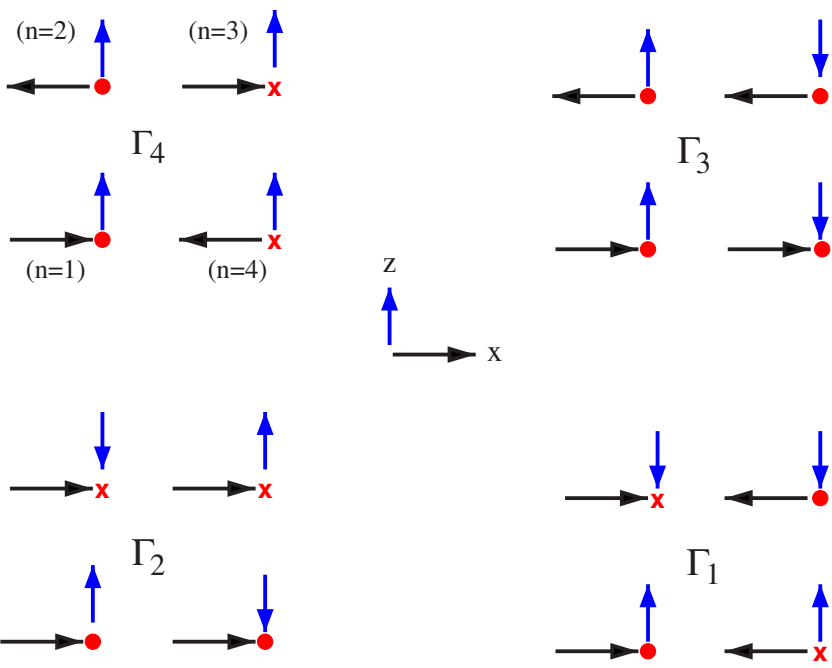

FIG. 16. (Color online) Schematic diagram of the spin wave functions within the unit cell of NVO for the various irreps. For simplicity, only the $\mathrm{Ni}$ spine sites at $\mathbf{r}=\mathbf{r}_{s, n}$ for $n=1,2,3,4$ (see Table II) are shown. The $x$ and $z$ axes are indicated and the positive $y$ axis is into the paper. (Filled circles represent spin components into the paper and crosses represent spin components out of the paper.) This figure is a pictorial representation of the data of Table IV. In the HTI phase, the spin distribution is that of $\Gamma_{4}$ within which the $x$ component is dominant.

$$
V_{\mathrm{e}-\mathrm{m}}=\sum_{\Gamma, \alpha, m} \epsilon_{\Gamma, \alpha, m}\left\langle\boldsymbol{\sigma}_{>}(\mathbf{q})\right\rangle \boldsymbol{\sigma}_{\Gamma}(-\mathbf{q}) Q_{\alpha, m}+\text { c.c. }
$$

where $\boldsymbol{\sigma}_{\Gamma}(\mathbf{q})$ represents a spin function having the symmetry of irrep $\Gamma$ and $\epsilon$ is a coefficient. Symmetry dictates that the only possible terms of this type have (a) $\Gamma=\Gamma_{2}$ in which case $\Gamma_{4} \times \Gamma_{2}$ transforms like $z$, so that in this term $\alpha=z$, and (b) $\Gamma=\Gamma_{1}$ in which case $\Gamma_{4} \times \Gamma_{1}$ transforms like $y$, so that in this term $\alpha=y$. Thus, we write

$$
\begin{aligned}
V_{\mathrm{e}-\mathrm{m}}= & \sum_{m} \boldsymbol{\epsilon}_{z}^{(m)}\left\langle\boldsymbol{\sigma}_{>}(\mathbf{q})\right\rangle \boldsymbol{\sigma}_{\Gamma_{2}}(-\mathbf{q}) Q_{z, m} \\
& +\sum_{m} \boldsymbol{\epsilon}_{y}^{(m)}\left\langle\boldsymbol{\sigma}_{>}(\mathbf{q})\right\rangle \boldsymbol{\sigma}_{\Gamma_{1}}(-\mathbf{q}) Q_{y, m}+\text { c.c. }
\end{aligned}
$$

Here, we see that magnons can only couple to $y$-like or $z$-like infrared active phonons. Then,

$$
\begin{aligned}
\left(\omega^{2}-\omega_{y, m}^{2}\right)\left\langle\left\langle Q_{y, m} ; Q_{y, m}\right\rangle\right\rangle= & 1+\boldsymbol{\epsilon}_{y}^{(m)}\left\langle\boldsymbol{\sigma}_{>}(\mathbf{q})\right\rangle\left\langle\left\langle\sigma_{\Gamma_{1}}(-\mathbf{q}) ; Q_{y, m}\right\rangle\right\rangle \\
& +\boldsymbol{\epsilon}_{y}^{(m) *}\left\langle\boldsymbol{\sigma}_{>}(\mathbf{q})^{*}\right\rangle\left\langle\left\langle\sigma_{\Gamma_{1}}(\mathbf{q}) ; Q_{y, m}\right\rangle\right\rangle .
\end{aligned}
$$

Similarly, the equations of motion with respect to the second argument yield

$$
\begin{aligned}
& \left(\omega^{2}-\omega_{y, m}^{2}\right)\left\langle\left\langle\sigma_{\Gamma_{1}}(\mathbf{q}) ; Q_{y, m}\right\rangle\right\rangle \\
& \quad=\epsilon_{y}^{(m)}\left\langle\boldsymbol{\sigma}_{>}(\mathbf{q})\right\rangle\left\langle\left\langle\sigma_{\Gamma_{1}}(\mathbf{q}) ; \sigma_{\Gamma_{1}}(-\mathbf{q})\right\rangle\right\rangle .
\end{aligned}
$$

In Fig. 16, we see that $\sigma_{\Gamma_{1}}$ has a $y$ component of spin which rotates the staggered moment (which is dominantly along the $x$ axis) of the unit cell. Therefore, this spin Green's function 
will intersect the lowest frequency magnon mode at frequency $\omega_{0}$. This same discussion also applies to the analogous treatment of the $z$-like phonon which couples to the $z$ component of $\sigma_{\Gamma_{2}}(\mathbf{q})$. For $n=1$ or $n=2$, we set

$$
\left\langle\left\langle\sigma_{\Gamma_{n}}(\mathbf{q}) ; \sigma_{\Gamma_{n}}(-\mathbf{q})\right\rangle\right\rangle=\frac{\langle S\rangle}{\omega^{2}-\omega_{0}^{2}},
$$

where $\langle S\rangle$ is a spin amplitude. In writing Eq. (217), we noted that the spin Green's function in Cartesian coordinates is a linear combination of raising and lowering spin Green's functions. Eventually, we are led to a solution which to leading order in the magnetoelectric interaction can be written as

$$
\left\langle\left\langle Q_{\alpha, m} ; Q_{\alpha, m}\right\rangle\right\rangle=\frac{1}{\omega^{2}-\omega_{\alpha, m}^{2}-\Sigma_{\alpha, m}},
$$

where

$$
\Sigma_{\alpha, m}=\frac{\mu_{\alpha, m}^{2}}{\omega^{2}-\omega_{0}^{2}},
$$

with $\mu_{\alpha, m}^{2}=2\langle S\rangle\left|\left\langle\sigma_{>}(\mathbf{q})\right\rangle \epsilon_{\alpha}^{(m)}\right|^{2}$. This form leads to mixing of the spin and phonon modes. The renormalized mode frequencies are given by the poles of the Green's function which occur at

$$
\widetilde{\omega}_{\alpha, m}^{2} \approx \omega_{\alpha, m}^{2}+\frac{\mu_{\alpha, m}^{2}}{\omega_{\alpha, m}^{2}-\omega_{0}^{2}} \approx \omega_{\alpha, m}^{2}+\frac{\mu_{\alpha, m}^{2}}{\omega_{\alpha, m}^{2}}
$$

and $^{87}$

$$
\widetilde{\omega}_{0}^{2}=\omega_{0}^{2}-\sum_{\alpha, m} \frac{\mu_{\alpha, m}^{2}}{\omega_{\alpha, m}^{2}-\omega_{0}^{2}} \approx \omega_{0}^{2}-\sum_{\alpha, m} \frac{\mu_{\alpha, m}^{2}}{\omega_{\alpha, m}^{2}},
$$

where $\alpha$ assumes the values $y$ and $z$ and we assumed that $\omega_{0} \ll \omega_{\alpha, m}$. The most important effect of this mixing is that it allows magnon absorption in an ac electric field. ${ }^{83}$ This is encoded in the Green's function

$$
\left\langle\left\langle\sigma_{\Gamma_{1}}(\mathbf{q}) ; Q_{\alpha, m}\right\rangle\right\rangle=-\frac{\epsilon_{\alpha, m}\left\langle\boldsymbol{\sigma}_{>}(\mathbf{q})\right\rangle\langle S\rangle}{\omega_{\alpha, m}^{2}\left(\omega^{2}-\omega_{0}^{2}\right)}
$$

when the ac electric field is along the $\alpha=y$ or $\alpha=z$ direction.

The above interpretation has to be modified for the system $\mathrm{Eu}_{0.75} \mathrm{Y}_{0.25} \mathrm{MnO}_{3}{ }^{88}$ As these authors discuss, the shift in the frequency of the optical phonon is too small to be consistent with the amount of its mixing with the magnon if one relies on a trilinear interaction of the form $V_{3} \sim \mu \sigma(\mathbf{q}) \sigma(-\mathbf{q}) Q$ (where $Q$ is a phonon amplitude), as we have assumed above. It is possible to avoid this inconsistency if one posits a quartic interaction of the form $V_{4} \sim \tau \sigma(\mathbf{q}) \sigma(-\mathbf{q}) Q Q$ and the sign of $\tau$ is such as to decrease the frequency of the optical phonon (thereby partially compensating its frequency shift proportional to $\mu^{2}$ associated with magnon-phonon mixing). Although $V_{4}$ is probably smaller than $V_{3}$, since it involves an additional derivative of the energy with respect to a phonon displacement, the frequency shift due to $V_{4}$ is proportional to $\tau$, whereas that due to $V_{3}$ is proportional to $\mu^{2} / \Delta E$, where $\Delta E$ is the difference in energy between the phonon and the magnon. Such a quartic interaction has been recently invoked by Fennie and Rabe in their treatment of magnonphonon interactions in $\mathrm{ZnCr}_{2} \mathrm{O}_{4} \cdot{ }^{89}$

\section{CONCLUSION}

In this paper, we have shown in detail how one can describe the symmetry of magnetic and magnetoelectric phenomena and have illustrated the technique by discussing several examples recently considered in the literature.

The principal results of this work are as follows.

(1) We discussed a method alternative to the traditional one (called representation analysis) for constructing allowed spin functions which describe incommensurate magnetic ordering. In many cases, this technique can be especially simple and does not require an understanding of group theory.

(2) For systems with a center of inversion symmetry, whether the simple method mentioned above or the more traditional representation formalism is used, it is essential to further include the restrictions imposed by inversion symmetry, as we pointed out previously. ${ }^{3-7}$

(3) We have illustrated this technique by applying it to systematize the magnetic structure analysis of several multiferroics many of which had not been analyzed using inversion symmetry.

(4) We discussed in all these systems how one introduces order parameters to characterize the spin structure. For incommensurate systems, these order parameters are inevitably complex because the origin of the incommensurate wave is either free or is only fixed by a very small locking energy.

(5) By considering several examples of multiferroics, we further illustrated the general applicability of the trilinear magnetoelectric coupling of the form $\boldsymbol{\sigma}(\mathbf{q}) \boldsymbol{\sigma}(-\mathbf{q}) P$, where $\boldsymbol{\sigma}(\mathbf{q})$ is the magnetic order parameter at wave vector $\mathbf{q}$ and $P$ is the uniform spontaneous polarization.

(6) The introduction of an order-parameter description of the spin structure has several advantages. First of all, since the transformation properties of the order parameters under the symmetry operations of the crystal are easy to analyze, it then is relatively simple to construct the explicit form of trilinear magnetoelectric coupling. This form allows us to predict how the temperature dependence of the spontaneous polarization is related to the various spin order parameters.

(7) Although our formulation is more complicated than those based on spiral magnetism, ${ }^{15,16}$ it allows us to discuss all multiferroics so far studied. In contrast, ${ }^{77}$ the discussions based on spiral magnetism are not general enough to discuss systems like RFMO, where the plane within which the spins rotate is perpendicular to the propagation vector of the magnetic state.

(8) We briefly discussed the implications of symmetry in assessing the role of various models proposed for multiferroics.

(9) We displayed the perturbation due to the interaction of three zone-center phonons, which leads to the mixing of Raman and infrared active phonon modes when the ferroelectric phase is entered. ${ }^{81}$ This interaction also leads to an anomalous contribution to the temperature dependence of the pho- 
non frequencies, which develops as the ferroelectric phase is entered.

(10) We presented a general analysis of the dynamics of magnon-phonon mixing based on symmetry.

\section{ACKNOWLEDGMENTS}

I acknowledge inspiration and advice from M. Kenzelmann who carried out several of the group theoretical calculations presented here. It should be obvious that this paper owes much to my other collaborators, especially G. Lawes, T. Yildirim, A. Aharony, O. Entin-Wohlman, C. Broholm, and A. Ramirez. I thank S.-H. Lee for providing me with the figure of $\mathrm{TbMn}_{2} \mathrm{O}_{5}$ and for insisting that I clarify various arguments. I am grateful to $\mathrm{G}$. Gasparovic for providing me with the figure of $\operatorname{RbFe}\left(\mathrm{MO}_{4}\right)_{2}$ and for access to his thesis. I am grateful to the authors of Ref. 10 for allowing me to reproduce their figure as Fig. 8 and I thank T. Kimura for attracting my attention to some recent references on $\mathrm{CuFeO}_{2}$. I thank J. Villain for calling my attention to some of the history of representation theory. I also wish to thank H. D. Drew for providing me with references invoked in Sec. V and for several instructive discussions of the experimental consequences of the magnetoelectric coupling.

\section{APPENDIX A: FORM OF EIGENVECTOR}

In this appendix, we show that the matrix $\mathbf{G}$ of the form of Eq. (86) [and this includes as a subcase the form of Eq. (83)] has eigenvectors of the form given in Eq. (87). Define $\mathbf{G}^{\prime} \equiv \mathbf{U}^{-1} \mathbf{G} \mathbf{U}$, where

$$
\mathbf{U}=\left[\begin{array}{ccccccc}
1 & 0 & 0 & 0 & 0 & 0 & 0 \\
0 & 1 & 0 & 0 & 0 & 0 & 0 \\
0 & 0 & 1 & 0 & 0 & 0 & 0 \\
0 & 0 & 0 & 1 / \sqrt{2} & i / \sqrt{2} & 0 & 0 \\
0 & 0 & 0 & 1 / \sqrt{2} & -i / \sqrt{2} & 0 & 0 \\
0 & 0 & 0 & 0 & 0 & 1 / \sqrt{2} & i / \sqrt{2} \\
0 & 0 & 0 & 0 & 0 & 1 / \sqrt{2} & -i / \sqrt{2}
\end{array}\right]
$$

We find that

$$
\mathbf{U}^{-1} \mathbf{G} \mathbf{U}=\left[\begin{array}{ccccccc}
a & b & c & \sqrt{2} \alpha^{\prime} & \sqrt{2} \alpha^{\prime \prime} & \sqrt{2} \xi^{\prime} & \sqrt{2} \xi^{\prime \prime} \\
b & d & e & \sqrt{2} \beta^{\prime} & \sqrt{2} \beta^{\prime \prime} & \sqrt{2} \eta^{\prime} & \sqrt{2} \eta^{\prime \prime} \\
c & e & f & \sqrt{2} \gamma^{\prime} & \sqrt{2} \gamma^{\prime \prime} & \sqrt{2} \kappa^{\prime} & \sqrt{2} \kappa^{\prime \prime} \\
\sqrt{2} \alpha^{\prime} & \sqrt{2} \beta^{\prime} & \sqrt{2} \gamma^{\prime} & g+\delta^{\prime} & \delta^{\prime \prime} & \mu^{\prime}+\nu^{\prime} & -\mu^{\prime \prime}-\nu^{\prime \prime} \\
\sqrt{2} \alpha^{\prime \prime} & \sqrt{2} \beta^{\prime \prime} & \sqrt{2} \gamma^{\prime \prime} & \delta^{\prime \prime} & g-\delta^{\prime} & \mu^{\prime \prime}-\nu^{\prime \prime} & \mu^{\prime}-\nu^{\prime} \\
\sqrt{2} \xi^{\prime} & \sqrt{2} \eta^{\prime} & \sqrt{2} \kappa^{\prime} & \mu^{\prime}+\nu^{\prime} & \mu^{\prime \prime}-\nu^{\prime \prime} & h+\rho^{\prime} & \rho^{\prime \prime} \\
\sqrt{2} \xi^{\prime \prime} & \sqrt{2} \eta^{\prime \prime} & \sqrt{2} \kappa^{\prime \prime} & -\mu^{\prime \prime}-\nu^{\prime \prime} & \mu^{\prime}-\nu^{\prime} & \rho^{\prime \prime} & h-\rho^{\prime}
\end{array}\right],
$$

where $\alpha^{\prime}$ and $\alpha^{\prime \prime}$ are the real and imaginary parts, respectively, of $\alpha$ and similarly for the other complex variables. Note that we have transformed the original matrix into a real symmetric matrix. Any eigenvector (which we denote $|R\rangle$ ) of the transformed matrix has real-valued components and thus satisfies the equation

$$
\mathbf{U}^{-1} \mathbf{G U}|R\rangle=\lambda_{R}|R\rangle,
$$

from which it follows that

$$
[\mathbf{G}] \mathbf{U}|R\rangle=\lambda_{R} \mathbf{U}|R\rangle,
$$

so that any eigenvector of $\mathbf{G}$ is of the form $\mathbf{U}|R\rangle$, where all components of $|R\rangle$ are real. If $|R\rangle$ has components $r 1, r 2, \ldots, r 7$, then

$$
\begin{gathered}
\mathbf{U}|R\rangle=\left[r 1, r 2, r 3,\left(r_{4}+i r_{5}\right) / \sqrt{2},\left(r_{4}-i r_{5}\right) / \sqrt{2},\right. \\
\left.\left(r_{6}+i r_{7}\right) / \sqrt{2},\left(r_{6}-i r_{7}\right) / \sqrt{2}\right],
\end{gathered}
$$

which has the form asserted. 
TABLE XXII. Character table for the double group of the wave vector. In the first line, we list the five classes of operators for this group. In the last line, we indicate the characters for the group $G$ which is induced by the $n$-dimensional reducible representation in the space of the $\alpha$ spin component of spins in a given Wyckoff orbit.

\begin{tabular}{lccccc}
\hline \hline Irrep & $E$ & $\pm m_{x}$ & $\pm m_{y}$ & $\pm m_{x} m_{y}$ & $-E$ \\
\hline$\Gamma_{a}$ & 1 & 1 & 1 & 1 & 1 \\
$\Gamma_{b}$ & 1 & -1 & 1 & -1 & 1 \\
$\Gamma_{c}$ & 1 & 1 & -1 & -1 & 1 \\
$\Gamma_{d}$ & 1 & -1 & -1 & 1 & 1 \\
$\Gamma_{2}$ & 2 & 0 & 0 & 0 & -2 \\
\hline$G$ & $n$ & 0 & 0 & 0 & $-n$ \\
\hline \hline
\end{tabular}

\section{APPENDIX B: IRREPS FOR TMO25}

In this appendix, we give the representation analysis for $\mathrm{TbMn}_{2} \mathrm{O}_{5}$ for wave vectors of the form $\left(\frac{1}{2}, 0, q\right)$, where $q$ has a nonspecial value. The operators we consider are $E, m_{x}, m_{y}$, and $m_{x} m_{y}$, as defined in Table XIII. Note that $m_{y}^{2}(x, y, z)$ $=(x+1, y, z)$, so that $m_{y}^{2}=-1$ for this wave vector. Thus, the above set of four operators does not actually form a group. Accordingly, we consider the double group which follows by introducing $-E$ defined by $m_{y}^{2}=-E,(-E)^{2}=E$, and $(-E) \mathcal{O}(-E)=\mathcal{O}$. Since addition has no meaning within a group, we do not discuss additive properties such as $(E)+(-E)=0$. Then, if we define $-\mathcal{O} \equiv(-E) \mathcal{O}$, we have the character table given in Table XXII.

The $\mathrm{Mn}^{4+}$ Wyckoff orbits contain two atoms and all the other orbits contain four atoms. In either case, we may consider separately an orbit and a single component, $x, y$, or $z$, of spin. So, the corresponding spin functions form a basis set of $n$ vectors, where $n=2$ for the single spin components of $\mathrm{Mn}^{4+}$ and $n=4$ otherwise. In each case, the operations involving $m_{x}$ and/or $m_{y}$ interchange sites and therefore have zero diagonal elements. Their character, which is their trace within this space of $n$ vectors, is therefore zero. On the other hand, $E$ and $-E$ give diagonal elements of +1 and -1 , respectively. So, their character (or trace) is $\pm n$ and we have the last line of the table for this reducible representation $G$.

In this character table, we also list (in the last line) the characters of these operations within the vector space of wave functions of a given spin component over a Wyckoff orbit of $n$ sites. Comparing this last line of the table to the character of the irreps, we see that $\mathbf{G}$ contains only the irrep $\Gamma_{2}$ and it contains this irrep $n / 2$ times. This means that for the system of three spin components over 12 sites, we have 36 complex components and these functions generate a reducible representation which contains $\Gamma_{2} 18$ times. If there were no other symmetries to consider, this result would imply that to determine the structure, one would have to fix the 18 complex-valued parameters. The two-dimensional representation can be realized by Eq. (125). The basis vectors which transform as the first and second columns, of the twodimensional representation are given in Table XXIII. One can check the entries of this table by verifying that the
TABLE XXIII. Spin functions (i.e., unit cell Fourier coefficients) determined by standard representation analysis without invoking inversion symmetry. The second and third columns give the functions which transform according to the first and second columns of the two-dimensional irrep. These coefficients are all complex parameters.

\begin{tabular}{|c|c|c|}
\hline Spin & $\boldsymbol{\sigma}_{1}$ & $\boldsymbol{\sigma}_{2}$ \\
\hline \multirow{3}{*}{$\mathbf{S}(\mathbf{q}, 1)$} & $r_{1 x}$ & $r_{2 x}$ \\
\hline & $r_{1 y}$ & $r_{2 y}$ \\
\hline & $r_{1 z}$ & $r_{2 z}$ \\
\hline \multirow{3}{*}{$\mathbf{S}(\mathbf{q}, 2)$} & $r_{2 x}$ & $r_{1 x}$ \\
\hline & $r_{2 y}$ & $r_{1 y}$ \\
\hline & $-r_{2 z}$ & $-r_{1 z}$ \\
\hline \multirow{3}{*}{$\mathbf{S}(\mathbf{q}, 3)$} & $r_{1 x}$ & $-r_{2 x}$ \\
\hline & $-r_{1 y}$ & $r_{2 y}$ \\
\hline & $-r_{1 z}$ & $r_{2 z}$ \\
\hline \multirow{3}{*}{$\mathbf{S}(\mathbf{q}, 4)$} & $r_{2 x}$ & $-r_{1 x}$ \\
\hline & $-r_{2 y}$ & $r_{1 y}$ \\
\hline & $r_{2 z}$ & $-r_{1 z}$ \\
\hline \multirow{3}{*}{$\mathbf{S}(\mathbf{q}, 5)$} & $r_{5 x}$ & $-r_{5 x}$ \\
\hline & $r_{5 y}$ & $-r_{5 y}$ \\
\hline & $r_{5 z}$ & $r_{5 z}$ \\
\hline \multirow{3}{*}{$\mathbf{S}(\mathbf{q}, 6)$} & $r_{5 x}$ & $r_{5 x}$ \\
\hline & $-r_{5 y}$ & $-r_{5 y}$ \\
\hline & $-r_{5 z}$ & $r_{5 z}$ \\
\hline \multirow{3}{*}{$\mathbf{S}(\mathbf{q}, 7)$} & $r_{6 x}$ & $-r_{6 x}$ \\
\hline & $r_{6 y}$ & $-r_{6 y}$ \\
\hline & $r_{6 z}$ & $r_{6 z}$ \\
\hline \multirow{3}{*}{$\mathbf{S}(\mathbf{q}, 8)$} & $r_{6 z}$ & $r_{6 x}$ \\
\hline & $-r_{6 y}$ & $-r_{6 y}$ \\
\hline & $-r_{6 z}$ & $r_{6 z}$ \\
\hline \multirow{3}{*}{$\mathbf{S}(\mathbf{q}, 9)$} & $r_{3 x}$ & $r_{4 x}$ \\
\hline & $r_{3 y}$ & $r_{4 y}$ \\
\hline & $r_{3 z}$ & $r_{4 z}$ \\
\hline \multirow{3}{*}{$\mathbf{S}(\mathbf{q}, 10)$} & $r_{4 x}$ & $r_{3 x}$ \\
\hline & $r_{4 y}$ & $r_{3 y}$ \\
\hline & $-r_{4 z}$ & $-r_{3 z}$ \\
\hline \multirow{3}{*}{$\mathbf{S}(\mathbf{q}, 11)$} & $r_{3 x}$ & $-r_{4 x}$ \\
\hline & $-r_{3 y}$ & $r_{4 y}$ \\
\hline & $-r_{3 z}$ & $r_{4 z}$ \\
\hline \multirow{3}{*}{$\mathbf{S}(\mathbf{q}, 12)$} & $r_{4 x}$ & $-r_{3 x}$ \\
\hline & $-r_{4 y}$ & $r_{3 y}$ \\
\hline & $r_{4 z}$ & $-r_{3 z}$ \\
\hline
\end{tabular}

effects of $m_{x}$ and $m_{y}$ on the vectors of this table are in conformity with Eq. (125).

However, after taking account of inversion symmetry, we have only 18 real-valued structural parameters of Table XVI to determine. 
${ }^{1}$ M. Fiebig, J. Phys. D 38, R123 (2005).

${ }^{2}$ T. Kimura, T. Goto, H. Shintani, K. Ishizka, T. Arima, and Y. Tokura, Nature (London) 426, 55 (2003).

${ }^{3}$ M. Kenzelmann, A. B. Harris, S. Jonas, C. Broholm, J. Schefer, S. B. Kim, C. L. Zhang, S.-W. Cheong, O. P. Vajk, and J. W. Lynn, Phys. Rev. Lett. 95, 087206 (2005).

${ }^{4}$ G. Lawes, A. B. Harris, T. Kimura, N. Rogado, R. J. Cava, A. Aharony, O. Entin-Wohlman, T. Yildrim, M. Kenzelmann, C. Broholm, and A. P. Ramirez, Phys. Rev. Lett. 95, 087205 (2005).

${ }^{5}$ A. B. Harris, J. Appl. Phys. 99, 08E303 (2006).

${ }^{6}$ M. Kenzelmann, A. B. Harris, A. Aharony, O. Entin-Wohlman, T. Yildirim, Q. Huang, S. Park, G. Lawes, C. Broholm, N. Rogado, R. J. Cava, K. H. Kim, G. Jorge, and A. P. Ramirez, Phys. Rev. B 74, 014429 (2006).

${ }^{7}$ A. B. Harris and G. Lawes, in The Handbook of Magnetism and Advanced Magnetic Materials, edited by H. Kronmuller and S. Parkin (Wiley, New York, 2007); arXiv:cond-mat/0508617 (unpublished).

${ }^{8}$ M. Kenzelmann, G. Lawes, A. B. Harris, G. Gasparovic, C. Broholm, A. P. Ramirez, G. A. Jorge, M. Jaime, S. Park, Q. Huang, A. Ya. Shapiro, and L. A. Demianets, Phys. Rev. Lett. 98, 267205 (2007).

${ }^{9}$ T. Goto, T. Kimura, G. Lawes, A. P. Ramirez, and Y. Tokura, Phys. Rev. Lett. 92, 257201 (2004)

${ }^{10}$ T. Kimura, J. C. Lashley, and A. P. Ramirez, Phys. Rev. B 73, 220401(R) (2006).

${ }^{11}$ K. Saito and K. Kohn, J. Phys.: Condens. Matter 7, 2855 (1995).

${ }^{12}$ I. Kagomiya, S. Matsumoto, K. Kohn, Y. Fukuda, T. Shoubu, H. Kimura, Y. Noda, and N. Ikeda, Ferroelectrics 286, 167 (2003).

${ }^{13}$ K. Taniguchi, N. Abe, T. Takenobu, Y. Iwasa, and T. Arima, Phys. Rev. Lett. 97, 097203 (2006).

${ }^{14}$ O. Heyer, N. Hollmann, I. Klassen, S. Jodlauk, L. Bohatý, P. Becker, J. A. Mydosh, T. Lorenz, and D. Khomskii, J. Phys.: Condens. Matter 18, L471 (2006).

${ }^{15}$ H. Katsura, N. Nagaosa, and A. V. Balatsky, Phys. Rev. Lett. 95, 057205 (2005).

${ }^{16}$ M. Mostovoy, Phys. Rev. Lett. 96, 067601 (2006).

${ }^{17}$ G. A. Smolenskii and I. E. Chupis, Sov. Phys. Usp. 25, 475 (1982)

${ }^{18}$ B. Dorner, J. Axe, and G. Shirane, Phys. Rev. B 6, 1950 (1972).

${ }^{19}$ R. A. Cowley, Adv. Phys. 29, 1 (1980).

${ }^{20}$ E. F. Bertaut, J. Phys. Colloq. 32, 462 (1971).

${ }^{21}$ J. Schweizer, J. Phys. IV 11, 9 (2001).

${ }^{22} \mathrm{~J}$. Rossat-Mignod, in Methods of Experimental Physics, edited by K. Skold and D. L. Price (Academic, New York, 1987), Vol. 23, Chap. 20, p. 69.

${ }^{23}$ J. Schweizer, C. R. Phys. 6, 375 (2005); 7, 823(E) (2006).

${ }^{24}$ P. G. Radaelli and L. C. Chapon, arXiv:cond-mat/0609087 (unpublished).

${ }^{25}$ J. Schweizer, J. Villain, and A. B. Harris, Eur. J. Phys. 38, 41 (2007).

${ }^{26}$ L. D. Landau and E. M. Lifshitz, Statistical Physics (Pergamon, London, 1958).

${ }^{27}$ M. Tinkham, Group Theory and Quantum Mechanics (McGrawHill, New York, 1964).

${ }^{28}$ J.-C. Tolédano and P. Tolédano, The Landau Theory of Phase Transitions (World Scientific, Singapore, 1987). In this reference, one can see the alternative approach to describing the free energy of incommensurate systems via a gradient expansion.
${ }^{29}$ For orthorhombic crystals, we will interchangeably refer to axes as either $x, y, z$, or $\mathbf{a}, \mathbf{b}, \mathbf{c}$.

${ }^{30}$ T. Nagamiya, in Solid State Physics, edited by F. Seitz and D. Turnbull (Academic, New York, 1967), Vol. 20, p. 346

${ }^{31}$ I. E. Dzialoshinskii, Sov. Phys. JETP 5, 1259 (1957).

${ }^{32}$ K. D. Bowers and J. Owen, Rep. Prog. Phys. 1, 304 (1955).

${ }^{33}$ A. J. C. Wilson, International Tables for Crystallography (Kluwer Academic, Dordrecht, 1995), Vol. A.

${ }^{34}$ Coordinates of positions within the unit cell are given as fractions of the appropriate lattice constants.

${ }^{35}$ E. E. Sauerbrei, F. Faggiani, and C. Calvo, Acta Crystallogr., Sect. B: Struct. Crystallogr. Cryst. Chem. 29, 2304 (1973).

${ }^{36}$ Often, these symmetry operations are labeled $h_{n}$, as in Refs. 33 or 37. Rather than use these symbols with no mnemonic value, we introduce the notation $m_{\alpha}$ for a mirror (or glide) plane which changes the sign of the $\alpha$ coordinate and $2_{\alpha}$ for a twofold rotation (or screw) axis parallel to the $\alpha$ direction.

${ }^{37}$ O. V. Kovalev, Representations of Crystallographic Space Groups: Irreducible Representations, Induced Representations and Corepresentations (Gordon and Breach, Amsterdam, 1993).

${ }^{38}$ G. Lawes, M. Kenzelmann, N. Rogado, K. H. Kim, G. A. Jorge, R. J. Cava, A. Aharony, O. Entin-Wohlman, A. B. Harris, T. Yildirim, Q. Z. Huang, S. Park, C. Broholm, and A. P. Ramirez, Phys. Rev. Lett. 93, 247201 (2004).

${ }^{39}$ Wave vectors are usually quoted in reciprocal lattice units (rlu's) so that $q_{x}$ in real units is $2 \pi q_{x} / a$. When $q$ is multiplied by a distance, obviously one has to either take $q$ in real units or take distances in inverse rlu's.

${ }^{40}$ A. B. Harris and J. Schweizer, Phys. Rev. B 74, 134411 (2006).

${ }^{41}$ When we "fix" the phase $\phi$ of a variable, it means that the variable is of the form $r e^{i \phi}$, where $r$ is real, but not necessarily positive.

${ }^{42}$ The fourth-order terms can also renormalize the quadratic terms, especially if one is far from the critical point. However, asymptotically close to the transition, this effect is negligible.

${ }^{43}$ H. Kawamura, Phys. Rev. B 38, 4916 (1988).

${ }^{44}$ A. D. Bruce and A. Aharony, Phys. Rev. B 11, 478 (1975).

${ }^{45}$ G. Lautenschlager, H. Weitzel, T. Vogt, R. Hock, A. Bóhm, M. Bonnet, and H. Fuess, Phys. Rev. B 48, 6087 (1993).

${ }^{46}$ Symmetry constraints on lock-in is discussed by Y. Park, K. Cho, and H.-G. Kim, J. Appl. Phys. 83, 4628 (1998).

${ }^{47}$ J. Blasco, C. Ritter, J. Garcia, J. M. de Teresa, J. Pérez-Cacho, and M. R. Ibarra, Phys. Rev. B 62, 5609 (2000).

${ }^{48}$ T. Kimura, G. Lawes, T. Goto, Y. Tokura, and A. P. Ramirez, Phys. Rev. B 71, 224425 (2005).

${ }^{49}$ R. Kajimoto, H. Yoshizawa, H. Shintani, T. Kimura, and Y. Tokura, Phys. Rev. B 70, 012401 (2004); 70, 219904(E) (2004).

${ }^{50}$ A. Munoz, J. A. Alonso, M. T. Casais, M. J. Martinez-Lope, J. L. Martinez, and M. T. Fernandez-Diaz, J. Phys.: Condens. Matter 14, 3285 (2002).

${ }^{51}$ A. Munoz, M. T. Casais, J. A. Alonso, M. J. Martinez-Lope, J. L. Martinez, and M. T. Fernandez-Diaz, Inorg. Chem. 40, 1020 (2001).

${ }^{52}$ H. W. Brinks, J. Rodriguez-Carvajal, H. Fjellvag, A. Kjekshus, and B. C. Hauback, Phys. Rev. B 63, 094411 (2001).

${ }^{53}$ G. Buisson, Phys. Status Solidi A 16, 533 (1973).

${ }^{54}$ G. Buisson, Phys. Status Solidi A 17, 191 (1973).

${ }^{55}$ L. C. Chapon, G. R. Blake, M. J. Gutmann, S. Park, N. Hur, P. G. Radaelli, and S.-W. Cheong, Phys. Rev. Lett. 93, 177402 (2004). 
${ }^{56}$ G. R. Blake, L. C. Chapon, P. G. Radaelli, S. Park, N. Hur, S.-W. Cheong, and J. Rodriguez-Carvajal, Phys. Rev. B 71, 214402 (2005).

${ }^{57}$ V. Heine, Group Theory in Quantum Mechanics (Pergamon, New York, 1960), p. 284. For an example, see A. J. Berlinsky and C. F. Coll III, Phys. Rev. B 5, 1587 (1972).

${ }^{58}$ L. C. Chapon, P. G. Radaelli, G. R. Blake, S. Park, and S.-W. Cheong, Phys. Rev. Lett. 96, 097601 (2006).

${ }^{59}$ A. B. Harris, arXiv:0707.1327 (unpublished).

${ }^{60}$ S. Mitsuda, H. Yoshizawa, N. Yaguchi, and M. Mekata, J. Phys. Soc. Jpn. 60, 1885 (1991).

${ }^{61}$ S. Mitsuda, M. Mase, K. Prokes, H. Kitizawa, and H. A. Katori, J. Phys. Soc. Jpn. 69, 3513 (2000).

${ }^{62}$ C. T. Prewitt, R. D. Shannon, and D. B. Rogers, Inorg. Chem. 10, 791 (1971)

${ }^{63}$ F. Ye, Y. Ren, Q. Huang, J. A. Fernandez-Baca, P. Dai, J. W. Lynn, and T. Kimura, Phys. Rev. B 73, 220404(R) (2006).

${ }^{64}$ N. Terada, S. Mitsuda, H. Ohsumi, and K. Tajima, J. Phys. Soc. Jpn. 75, 023602 (2006).

${ }^{65}$ N. Terada, Y. Tanaka, Y. Tabata, K. Katsumata, A. Kikkawa, and S. Mitsuda, J. Phys. Soc. Jpn. 75, 113702 (2006).

${ }^{66}$ N. Terada, T. Kawasaki, S. Mitsuda, H. Kimura, and Y. Noda, J. Phys. Soc. Jpn. 74, 1561 (2005).

${ }^{67}$ G. Gasparovic, Ph.D. thesis, Johns Hopkins University, 2004.

${ }^{68}$ L. E. Svistov, A. I. Smirnov, L. A. Prozorova, O. A. Petrenko, L. N. Demianets, and A. Y. Shapiro, Phys. Rev. B 67, 094434 (2003).

${ }^{69}$ L. E. Svistov, A. I. Smirnov, L. A. Prozorova, O. A. Petrenko, A. Micheler, N. Buttgen, A. Y. Shapiro, and L. N. Demianets, Phys. Rev. B 74, 024412 (2006).

${ }^{70}$ G. A. Jorge, C. Capan, F. Ronning, M. Jaime, M. Kenzelmann, G. Gasparovic, C. Broholm, A. Ya. Shapiro, and L. A. Demianets, Physica B 354, 297 (2004).

${ }^{71}$ D. Frohlich, St. Leute, V. V. Pavlov, and R. V. Pisarev, Phys. Rev. Lett. 81, 3239 (1998).

${ }^{72}$ T. A. Kaplan and N. Menyuk, Philos. Mag. (to be published).
${ }^{73}$ I. Dzialoshinskii, J. Phys. Chem. Solids 4, 241 (1958); T. Moriya, Phys. Rev. 120, 91 (1960).

${ }^{74}$ It is true that the coefficient $r_{\gamma}$ can depend on temperature and it would be of the form $r_{\gamma}(T) \approx r_{\gamma}\left(T_{c}\right)+r_{1}\left(T-T_{c}\right)$. There is no reason to think that $r_{\gamma}\left(T_{c}\right)$ would be unusually small, and we therefore have the desired result as long as we are not far from the critical temperature $T_{c}$.

${ }^{75}$ A. B. Harris, T. Yildirim, A. Aharony, and O. Entin-Wohlman, Phys. Rev. B 73, 184433 (2006).

${ }^{76}$ J. J. Betouras, G. Giovannetti, and J. van den Brink, Phys. Rev. Lett. 98, 257602 (2007).

${ }^{77}$ M. Kenzelmann and A. B. Harris, Phys. Rev. Lett. (to be published); arXiv:cond-mat/0610471 (unpublished).

${ }^{78}$ I. A. Sergienko and E. Dagotto, Phys. Rev. B 73, 094434 (2006).

${ }^{79}$ I. A. Sergienko, C. Sen, and E. Dagotto, Phys. Rev. Lett. 97, 227204 (2006)

${ }^{80}$ D. V. Efremov, J. van den Brink, and D. Khomskii, Physica B 359, 1433 (2005)

${ }^{81}$ R. Valdes Aguilar, A. B. Sushkov, S. Park, S.-W. Cheong, and H. D. Drew, Phys. Rev. B 74, 184404 (2006).

${ }^{82}$ A. Pimenov, A. A. Mukhin, V. Yu. Ivanov, V. D. Travkin, A. M. Balbashov, and A. Loidl, Nat. Phys. 2, 97 (2006).

${ }^{83}$ H. Katsura, A. V. Balatsky, and N. Nagaosa, Phys. Rev. Lett. 98, 027203 (2007).

${ }^{84}$ A. Pimenov, T. Rudolf, F. Mayr, A. Loidl, A. A. Mukhin, and A. M. Balbashov, Phys. Rev. B 74, 100403(R) (2006).

${ }^{85}$ A. B. Sushkov, R. V. Aguilar, S. Park, S.-W. Cheong, and H. D. Drew, Phys. Rev. Lett. 98, 027202 (2007).

${ }^{86}$ D. N. Zubarev, Sov. Phys. Usp. 3, 320 (1960).

${ }^{87}$ To get this more or less obvious result, which involves a sum over optical phonon modes, requires a more delicate analysis than given here.

${ }^{88}$ R. Valdés Aguilar, A. B. Sushkov, C. L. Zheng, Y.-J. Choi, S.-W. Cheong, and H. D. Drew, arXiv:0704.3632 (unpublished).

${ }^{89}$ C. J. Fennie and K. M. Rabe, Phys. Rev. Lett. 96, 205505 (2006). 